\title{
THE PREPARATION OF FUNCTIONAL SURFACES
}

\author{
A Thesis \\ presented to \\ the Faculty of California Polytechnic State University, \\ San Luis Obispo
}

\begin{abstract}
In Partial Fulfillment
of the Requirements for the Degree

Master of Science in Polymers and Coatings (Chemistry Department)
\end{abstract}

by

Philip Thomas Dirlam

June 2011 
(C) 2011

Philip Thomas Dirlam

ALL RIGHTS RESERVED 


\title{
COMMITTEE MEMBERSHIP
}

TITLE: $\quad$ THE PREPARATION OF FUNCTIONAL SURFACES

\author{
AUTHOR: Philip Thomas Dirlam
}

DATE SUBMITTED: June 2011

COMMITEE CHAIR: $\quad$ Dr. Philip J. Costanzo, Assistant Professor

COMMITTEE MEMBER: Dr. Jeffrey Pyun, Associate Professor University of

Arizona

COMMITTEE MEMBER: $\quad$ Dr. Raymond H. Fernando, Professor 


\section{ABSTRACT \\ The Preparation of Functional Surfacs \\ Philip Thomas Dirlam}

Diels-Alder chemistry was utilized to manipulate the surface energy of glass substrates in reversible manner. Glass slides and capillaries were functionalized with hydrophobic dieneophiles resulting in a non-wetting surface. A retro Diels-Alder reaction facilitated by the thermal treatment of the surface's function to cleave the hydrophobic dieneophile and resulted in the fabrication of a hydrophilic surface. Contact angle (CA) measurements were used as preliminary measurements for monitoring the changes in surface energy exhibited during the initial hydrophobic state $\left(\mathrm{CA}-70 \pm 3^{\circ}\right)$, after attachment of the dieneophile creating a hydrophobic state $\left(\mathrm{CA}-101 \pm 9^{\circ}\right)$ followed by reestablishment of the hydrophilic state $\left(\mathrm{CA}-70 \pm 6^{\circ}\right)$ upon cleavage of the DielsAlder adduct. The treatments developed on flat glass surfaces were transferred to glass capillaries, with effective treatment confirmed by fluid column measurements. Effective flow gating was developed in the capillaries via patterning of the surface with hydrophilic/hydrophobic regions. Finally, attempts to create self-pressurizing capillaries were unsuccessful due to pronounced contact angle hysteresis for the hydrophobic surface treatment.

Indium-tin oxide (ITO) substrates were functionalized with successive surface intiated atom transfer radical polymerization (SI-ATRP) and electropolymerization. A novel hybrid styrenic/thiophene monomer (ProDOT-Sty) was synthesized and employed in the polymerization events. This unique monomer and combination of polymerization 
methods allowed for the templation of electropolymerized poly $(3,4-$

alkyleneoxythiophene) brushes by first creating a poly(styrene) backbone via SI-ATRP. An ITO electrode functionalized with poly(ProDOT-Sty) brushes grafted from the ITO surface via SI-ATRP was analyzed via cyclic voltammetry which clearly indicated the electropolymerization event beginning at approximately $+0.7 \mathrm{~V}$ vs $\mathrm{Fc} / \mathrm{Fc}^{+}$. Photo patterning of the phosphonic acid ATRP initiator immobilized on the ITO surface was undertaken in order to create a surface that would limit growth of the polymer species to a patterned area for facile film brush thickness characterization via atomic force microscopy (AFM) at a later time. This was accomplished via lithography with ultraviolet radiation (UV) and was confirmed via scanning electron microscopy (SEM).

A nanohetero structure composed of platinum tipped cadmium selenide seeded, cadium sulfide nanorods (CdSe@CdS-Pt NRs). CdSe quantum dots (QDs) with variable sizes were prepared by adjusting reaction temperatures and times. CdS nanorods were then grown utilizing the CdSe QDs as seeds. Various lengths of the CdSe@CdS NRs were produced that ranged from $\sim 25 \mathrm{~nm}$ to $\sim 135 \mathrm{~nm}$. Investigation of the influence of the various synthetic conditions of the nanorod synthesis led to the conclusion that the ratio of $\mathrm{CdSe}$ seeds to $\mathrm{Cd}$ and $\mathrm{S}$ precursors could be manipulated in order to influence the length to which the nanorods grew. Pt tips were attached to an end of the CdSe@CdS nanorods as photocatalytic hydrogen production sites. TEM was utilized to characterize the different types of nanoparticles at each stage of assembly. 


\section{ACKNOWLEDGEMENTS}

First and foremost I would like to acknowledge my advisor and mentor Dr. Phil Costanzo. I was fortunate to be his first student at Cal Poly, and as such was privileged to learn many of my skills in the chemical sciences from him firsthand. He has afforded me invaluable opportunities in study at laboratories across the country that have allowed me to become the chemist I am. Beyond academia he has been a mentor through the processes of maturing socially, morally and mentally throughout my collegiate experience and has left me prepared for my future journeys.

Second I would like to acknowledge my advisor Dr. Jeff Pyun. He welcomed me into his research group at the University of Arizona at the end of my $4^{\text {th }}$ year at Cal Poly. After a short time in his group he was gracious enough to assign me my own project that pushed my abilities as an experimental chemist on a day-to-day basis. He taught me how to maximize the impact of the synthetic skillset I had developed so far and encouraged me to develop further. 
TABLE OF CONTENTS

Page

LIST OF TABLES

xii

LIST OF FIGURES xiii

LIST OF SCHEMES xvi

1. INTRODUCTION

1.1. INTERFACES 1

1.2. SURFACE TENSION AND SURFACE ENERGY 1

1.2.1. MEASURING SURFACE TENSION AND SURFACE 2 ENERGY VIA CONTACT ANGLE

1.2.2. SURFACE ROUGHNESS AND HETEROGENEITY 4

1.2.2.1. CONTACT ANGLE HYSTERSIS 4

1.2.2.2. CONTACT ANGLE HYSTERESIS DUE TO 5

SURFACE ROUGHNESS AND HETEROGENEITY

1.2.2.3. INFLUENCE OF SURFACE ROUGHNESS ON

MEAN CONTACT ANGLE

1.3. COLLOIDS 8

1.4. STATEMENT OF PURPOSE 10

1.5. REFERENCES 11

2. CONTROLLING SURFACE ENERGY AND WETABILITY WITH 13 
DIELS-ALDER CHEMISTRY

$\begin{array}{ll}\text { 2.1. INTRODUCTION } & 13\end{array}$

2.1.1. MOTIVATION AND BRIEF REVIEW OF PREVIOUS 13 WORKS

$\begin{array}{ll}\text { 2.1.2. THEORETICAL MODELING } & 14\end{array}$

2.1.3. EXPERIMENTAL STRATEGY 17

$\begin{array}{lr}\text { 2.2. RESULTS AND DISCUSSION } & 18\end{array}$

2.2.1. FLAT SURFACE MODIFICATION AND SUBSEQUENT 18 CHARACTERIZATION

2.2.2. DIELS-ALDER OPTIMIZATION 22

2.2.3. CAPILLARY MODIFICATION AND CHARACTERIZATION 23

2.2.4. PATTERNED HYDROPHILIC/HYDROPHOBIC 25 CAPILLARIES

2.2.4.1. PREPARATION OF PATTERNED 25 HYDROPHOBIC/HYDROPHILIC CAPILLARIES

2.2.4.2 DEMONSTRATION OF FLOW GATING 26

2.2.4.3 FLOW EXPERIMENTS 27

2.3. CONCLUSIONS 33

2.4. EXPERIMENTAL 34

2.4.1. MATERIALS 34

2.4.2. INSTRUMENTATION 34

2.4.3. SYNTHETIC PRERPATIONS 
2.4.3.1. SYNTHESIS OF CARBOXY MALEIMIDE 35

2.4.3.2. SYNTHESIS OF FLUORINATED MALEIMIDE 36

2.4.4. TYPICAL METHODOLOGY FOR SURFACE PREP 37

2.4.5. CAPILLARY EXPERIMENTS 38

$\begin{array}{lll}\text { 2.4.5.1. STATIC FILL } & 38\end{array}$

2.4.5.2. DYNAMIC FILL 38

$\begin{array}{lll}\text { 2.4.5.3. PURGING } & 39\end{array}$

2.5. REFERENCES 40

3. ALIGNED POLY(3,4-ALKYLENEOXYTHIOPHEN) BRUSHES ON 41 INDIUM TIN OXIDE FROM SURFACE INTIATIED ATOM TRANSFER RADICAL POLYMERIZATION AND SUBSEQUENT ELECTROPOLYMERIZATION

3.1. INTRODUCTION 41

3.1.1. MOTIVATION 41

3.1.2. CURRENT TECHNOLOGIES 42

3.1.2.1. INORGANIC SOLAR CELLS 42

3.1.2.2. ORGANIC PHOTOVOLTAICS 43

3.1.2.2.1. ITO TRANSPARENT ANODE 44

3.1.2.2.2. PHOTOACTIVE LAYER 45

3.1.2.2.3. CONDUCTIVE POLYMER LAYER 48

3.1.3. EXPERIMENTAL STRATEGY 49

3.2. RESULTS AND DISCUSSION 50 
3.2.1. STEPWISE SURFACE MODIFICATION OF ITO 43

3.2.2. PATTERNING OF ATRP INITIATOR ON ITO 52

3.2.3. ELECTROPOLYMERIZATION OF PRODOT SIDE CHAIN 55

3.3. CONCLUSIONS 55

3.4. EXPERIMENTAL 56

3.4.1. MATERIALS 56

3.4.2. INSTRUMENTATION 56

3.4.3. SYNTHETIC PREPARATIONS 57

3.4.3.1. SYNTHESIS OF PRODOT-OH

3.4.3.2. SYNTHESIS OF PRODOT-STY $\quad 58$

3.4.3.3. ATRP OF PRODOT-STY $\quad 59$

3.4.4. SURFACE MODIFICATION 59

3.4.4.1. ITO MODIFICATION WITH ATRP INTIATOR 59

3.4.4.2. SI-ATRP OF PRODOT-STY 60

3.5. REFERENCES 61

4. PREPARATION OF FUNCTIONAL SEMICONDUCTOR/METAL 63 NANOSTRUCTURES

4.1. INTRODUCTION 63

4.1.1. MOTIVATION 63

4.1.2. SEMICONDUCTOR/METAL NANOPARTICLE SYNTHESIS 64

4.1.3. CADMIUM SULFIDE/PT PHOTOCHEMICAL SYSTEM 65

4.1.4. QUANTUM CONFINEMENT 65

4.1.5. EXPERIMENTAL STRATEGY 66 
4.2. RESULTS AND DISCUSSION 67

4.2.1. CDSE QUANTUM DOT SYNTHESIS 67

4.2.2.CDSE@CDS NANOROD SYNTHESIS 70

4.2.3 PT TIPPED CDSE@CDS NANOROD SYNTHESIS 73

$\begin{array}{ll}4.3 \text { CONCLUSIONS } & 74\end{array}$

$\begin{array}{ll}\text { 4.4 EXPERIMENTAL } & 74\end{array}$

4.4.1. MATERIALS 74

$\begin{array}{ll}\text { 4.4.2 INSTRUMENTATION } & 74\end{array}$

4.4.3. NANOPARTICLE SYNTHESIS $\quad 75$

4.4.3.1. CADMIUM SELENIDE QUANTUM DOTS 75

4.4.3.2.CDSE@CDS NANORODS SYNTHESIS 75

4.4.3.3. PT TIPPING OF NANORODS 77

$\begin{array}{ll}\text { 4.5 REFERENCES } & 77\end{array}$

$\begin{array}{ll}\text { 5. CONCLUSIONS } & 79\end{array}$ 
LIST OF TABLES $\quad$ Page

1.1 Types of Colloidal Systems 8

2.1 Contact Angle Measuremens of Glass Slides and Capillaries at Various 21 States of Functionalization

3.1 Theoretical Energy Potential of Various Renewable Energy Ressources 42

4.1 Synthetic Conditions With Varied QD concentrations for Preparation of 71 CdSe@CdS Nanorods

4.2 Synthetic Conditions With Varied $\mathrm{CdO}$ concentrations for Preparation of 71 CdSe@CdS Nanorods

4.3 Synthetic Conditions With Varied S:TOP Injection Temperations for 71 Preparation of CdSe@CdS Nanorods

4.4 Synthetic conditions for Various Preparations of CdSe@CdS Nanorods 76 
1.1 Schematic of the molecular structure of a liquid-gas interface depicting the different number of attractive forces in action on a molecule at the surface compared to in the bulk.

1.2 Schematic depicting a cross section of the edge of a liquid drop on a solid surface with contact angle $(\Theta)$ and the surface tension of the liquid-gas $\left(\gamma_{\mathrm{L}}\right)$, solid-gas $\left(\gamma_{\mathrm{G}}\right)$, and solid-liquid $\left(\gamma_{\mathrm{SL}}\right)$

1.3 Schematic of a drop advancing over a surface with a micro scale protrusion. The top diagrams depict the situation as would appear to the eye while the bottom diagrams schematically illustrats the details of the situation at the micro scale leading to line pinning.

1.4 Schematic illustrating the line pinning of a liquid droplet advancing on a chemically heterogeneous surface from a region of lower $\gamma$ to higher $\gamma$.

1.5 Schematic depiction of contact angle amplification of a nonwetting surface (A) and a wetting surface (B) due to surface roughness in accordance with the Wenzel equation.

1.6 Tunable features of a nanoparticle.

2.1 Schematic of a horizontally inclined capillary that defines problem.

2.2 Reversible Diels-Alder adduct whose formation is temperature dependent.

2.3 XPS analysis of functionalized surfaces corresponding to those listed in Table 1.

2.4 Optical micrograph of contact angle measurements of (A) furan functionalized slide (Surface 2) and (B) fluorinated functionalized slide (Surface 3).

2.5 Various Diels-Alders systems that were utilized to prepare thermo-responsive surfaces.

2.6 Optical micrograph of capillaries at various stages of functionalization filled with $\mathrm{H}_{2} \mathrm{O}$ containing fluorescein taken under UV conditions. Letters refer to capillaries as denoted in Table 1. Numerical values represent the height of capillary fill. Each grid block represents $0.5 \mathrm{~cm}$. [Note fluorescent $\mathrm{H}_{2} \mathrm{O}$ was utilized to aid the eye. It has no effect on capillary action.] 

internal fluid slug (dyed $\mathrm{H}_{2} \mathrm{O}$ ). The red line segment indicates the hydrophobic length of the capillary, while the blue line segment indicates the hydrophilic region. Gravity is oriented downward in both images. Inset schematics represent slug interface characteristics for each case.

Schematic representation of the dynamic capillary filling experiment. Actual experiment was conducted by raising the reservoir, which would have the same effect as lowering the capillary. where $\mathrm{H} 1$ is the nominal vertical capillary wetting height for strongly hydrophilic capillary, $\mathrm{H} 2$ is the nominal vertical capillary wetting height for weakly hydrophobic capillary, $y$ is the vertical position of bottom of capillary relative to reservoir height, $h$ is the stable, instantaneous position of fluid column meniscus relative to reservoir height, and L is total capillary length.

2.10 Optical micrographs of dynamic capillary fill experiment.

A) Hydrophilic capillary analogous to Surface 1 and Capillary C/E; B) Patterned capillary (bottom half hydrophilic, top half hydrophobic), C) Hydrophobic calillary analogous to Surface 2 and Capillary D.

2.11 Dynamic capillary fill experiments comparing capillary action in reference to the height of the reservoir against the depth of the capillary.

2.12 Left: Schematic of purge model; Right: Resulting purge time as a function of contact angle and diameter, for a water-filled capillary with an aspect ratio of 100 .

2.13 Schematic representations of the self-purging capillary experiments.

3.1 Basic OPV cell structure.

3.2 Schematics of bilayer (a), disordered bulk heterojunction (b) and ordered bulk heterojunction (c) photoactive layers.

3.3 Examples of organic semi-conductors used in organic solar cells.

3.4 Morphology of PEDOT:PSS transparent conductive polymer layer cast on an indium tin-oxide substrate. 
3.5 Design of conductive poly(thiophene) layer from SI-ATRP and subsequent electropolymerization. Blue constituents represent the poly(styrene) backbone and red species represent the aligned, conductive poly(thiophene) chains.

3.6 Expanded XPS spectrum of Br 3d of ATRP initiator functionalized ITO over 90 minutes of UV irradiation. Reproduced from Kim et. al. ${ }^{16}$

3.7 SEM micrographs of photopatterned ITO

3.8 Cyclic voltammogram of poly(ProDOT-Sty) grafted from ITO referenced against $\mathrm{Fc} / \mathrm{Fc}^{+}$

4.1 Schematic illustration of the Co-CdSe@CdS-Pt nanorod structure illustrating theoretical photoinduced redox chemisty.

4.2 TEM micrographs of CdSe QDs with diameters of a) $~ 3.8$ $\mathrm{nm}$ and b) $\sim 2.6 \mathrm{~nm}$

4.3 Fluorescence spectra of the blue-green QDs and yellowgreen QDs.

4.4 TEM microcraph of nanorods with a length of $27.82 \pm 0.98$ nm (Sample 17)

4.5 TEM microcraph of nanorods with a length of $105.2 \pm 15.2$ nm (Sample 26)

4.6 TEM micrograph of CdSe@CdS-Pt nanorods where the dark circles are indicitave of the Pt tips 


\section{LIST OF SCHEMES}

2.1 Synthetic route for preparation of Diels-Alder 19 functionalized glass slides

2.2 Synthetic route for preparation of fluorinated 19 maleimide.

3.1 Preparation of 4-(bromomethyl)benzylphosphonic 50 acid

3.2 Synthetic Route for Preparation of Aligned Poly(3,4- 51 alkyleneoxythiophene) Brushes from Indium Tin Oxide Surfaces.

3.3 Synthetic Route for Preparation of ProDOT-Sty 51

3.4 Photopatterning of ATRP initiator functionalized ITO 52

4.1 Preparation of CdSe Quantum Dots 68

4.2 Preparation of CdSe@CdS Nanorods 70

4.3 Assembly of CdSe@CdS-Pt Nanorods 73 


\section{Introduction}

\subsection{Interfaces}

The separation of two distinct phases from each other results in the creation of an interface. With the consideration of solid, liquid and gas phases, the three states of matter most commonly encountered, three combinations of interfaces are generated: solidliquid, solid-gas, and liquid gas. These three types of interfaces are more specifically referred to as surfaces. ${ }^{1}$ The term interface is more general and is able to describe the liquid-liquid boundary created from the interaction of two immiscible liquids as well as solid-solid separations. Gas-gas interfaces do not exist as gaseous matter mixes. ${ }^{2}$

\subsection{Surface Tension and Surface Energy}

The most fundamental physical quantity in surface science is surface tension. At the most basic level, surface tension can be described as the work required to increase the area of a surface in an isothermal and reversible manner as shown by Equation 1.1 where the proportionality constant $(\gamma)$ is surface tension which is commonly reported in $\mathrm{mN} / \mathrm{m} .^{1-}$

${ }^{3}$ It is also commonly defined as tensor force that acts perpendicularly to a line of unit length on a surface. ${ }^{4}$

$$
d W=\gamma \cdot d A
$$

The interpretation of surface tension at the molecular level leads one to the elucidation of the definition of surface energy. This interpretation is developed from the fact that it is energetically favorable for molecules of a certain substance to be completely surrounded by other molecules of that substance, usually referred to as have the 


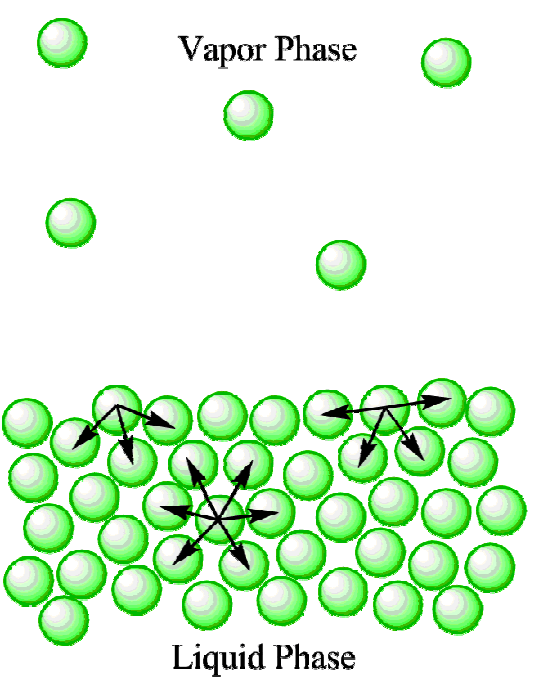

Figure 1.1 Schematic of the molecular structure of a liquid-gas interface depicting the different number of attractive forces in action on a molecule at the surface compared to in the bulk.

maximum number of nearest neightbors. ${ }^{3}$ These molecules attract one another via interactions such as van der Waals forces and hydrogen bonding. The existence of a condensed phase is in itself proof of these attractive forces between molecules because without such attraction there would only be a vapor phase. ${ }^{2}$ At a surface or interface molecules are only partially surrounded by other molecules thus the number of contiguous molecules is lessened compared to that of the bulk as depicted in Figure 1.1. This is an energetically unfavorable event. Thus to bring a molecule from the bulk to the surface work has to be done and the energy expended during this process is referred to as surface energy which is synonymously denoted $\gamma$ and commonly reported in $\mathrm{J} / \mathrm{m}^{2}$.

As a result of the interfacial tension exhibited at the surface of a liquid that is not in contact with another condensed phase, such as a water droplet in air, the system assumes a shape with minimum surface area. This shape is that of a sphere and as such, there are square or elliptical water droplets. The water droplet retains this shape 
conformation in order to minimize the area-to-volume ratio and subsequently the number of molecules, in the unfavorable energetic state previously described, at the surface.

\subsubsection{Measuring Surface Tension and Surface Energy via Contact Angle}

The measurement of surface tension requires the introduction of the concept of wetting and subsequently the contact angle. Wetting fundamentally includes all incidences involving contacts between a triplet of phases, two of which are fluid (gaseous or liquid). ${ }^{2}$ The most familiar circumstance of wetting occurs when a solid surface is being wetted by a liquid in a gaseous environment.

The basal quantitative explication of wetting phenomenon is given by Young's equation. This formula relates the contact angle $(\Theta)$ to the interfacial tensions of the liquid-gas $\left(\gamma_{\mathrm{L}}\right)$, solid-gas $\left(\gamma_{\mathrm{G}}\right)$, and solid-liquid $\left(\gamma_{\mathrm{SL}}\right)$ as shown in Equation $1.2^{5}$ and

$$
\gamma_{L} \cdot \cos \Theta=\gamma_{S}-\gamma_{S L}
$$

depicted by Figure 1.2. When the interfacial tension of the bare solid is greater than that of the solid-liquid interface then the right hand side of Young's equation becomes positive, dictating that the cosine of the contact angle is positive and ultimately that the contact angle is less that $90^{\circ}$. When a contact angle less than $90^{\circ}$ is observed the surface is described as partially wetting with respect to the liquid. When the opposite case is true and the solid-liquid interface is energetically less favorable than that of the bare solid $\left(\gamma_{S}\right.$ $<\gamma_{\mathrm{SL}}$ ) the contact angle will exceed $90^{\circ}$ yielding a "non-wetting" surface. 


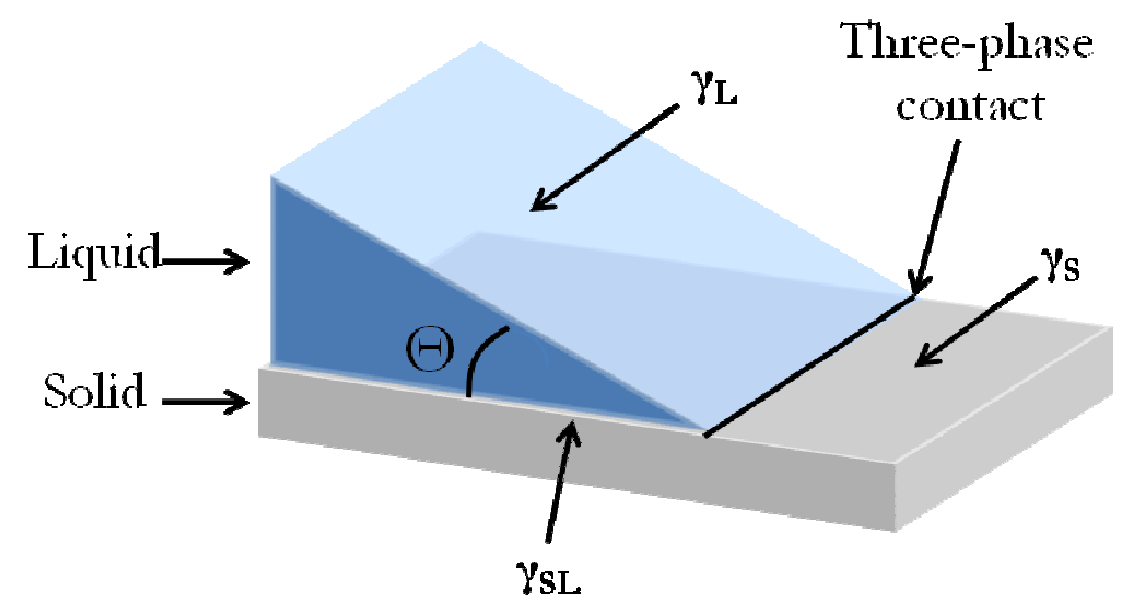

Figure 1.2 Schematic depicting a cross section of the edge of a liquid drop on a solid surface with contact angle $(\Theta)$ and the surface tension of the liquid-gas $\left(\gamma_{L}\right)$, solid-gas $\left(\gamma_{\mathrm{G}}\right)$, and solid-liquid $\left(\gamma_{\mathrm{SL}}\right)$

Once the contact angle is measured the observed measurement is fitted with a contour calculated via the Young-Laplace equation (Equation 1.3) where $\Delta \mathrm{P}$ is the pressure difference between the two phases and $\mathrm{R}_{1}$ and $\mathrm{R}_{2}$ are the two principle radii. Through this fit the surface tension is obtained. ${ }^{2}$

$$
\Delta \mathrm{P}=\gamma \cdot\left(1 / \mathrm{R}_{1}+1 / \mathrm{R}_{2}\right)
$$

\subsubsection{Surface Roughness/Heterogeneity}

\subsubsection{Contact Angle Hysteresis}

Hysteresis generally refers to a system that exhibits a degree of path dependence. In terms of contact angle this can be illustrated by again considering a drop of liquid on a planar, solid surface. If the contact angle is measured as the volume of the drop increases and the wetting line advances the advancing contact angle $\left(\Theta_{\mathrm{adv}}\right)$ is observed. Conversely if the contact angle is measured as the volume of the drop is decreased and the wetting 
line recedes the receding contact angle $\left(\Theta_{\text {rec }}\right)$ is resultant. Typically the advancing contact angle is observed to be notably higher than that of the receding contact angle . The difference between the two, $\Theta_{\mathrm{adv}}-\Theta_{\mathrm{rec}}$, is referred to as the contact angle hysteresis. ${ }^{6}$ The phenomenon of contact angle hysteresis greatly complicates the mathematical treatments that profile contact angle measurements and can be attributed to a number of different causes, most notably surface roughness and surface heterogeneity.

\subsection{Contact Angle Hysteresis Due to Surface Roughness and Heterogeneity}

To the naked eye many surfaces appear to possess an ideal, perfectly smooth surface, but below the microscopic scale every surface possesses some degree of inherent roughness. ${ }^{2}$ Figure 1.3 depicts a solid surface with a microscopic projection over which a drop of liquid with contact angle of $\Theta=90^{\circ}$ ( Figure 1.3A) is advancing. When the



Figure 1.3 Schematic of a drop advancing over a surface with a micro scale protrusion. The top diagrams depict the situation as would appear to the eye while the bottom diagrams schematically illustrats the details of the situation at the micro scale leading to line pinning. 
droplet encounters the protrusion it will advance until it reestablishes its contact angle of $90^{\circ}$ (Figure 1.3B) after which its spreading will be hindered, a phenomenon commonly referred to as "line pinning" in reference to the movement of the three-phase contact line being impeded. At the macroscopic level, this situation will produce an apparent contact angle that is larger than $90^{\circ}$ (Figure 1.3C) and will persist until after the droplet overcomes the protrusion. $^{7}$ As roughness can be considered as a densely packed gathering of protrusions this happening will recur as the drop overcomes each new protrusion it encounters. When the liquid recedes the analogous situation, where the character of the contact angle is amplified, occurs and thus hysteresis is observed. ${ }^{8}$

Chemically heterogeneous surfaces also function to facilitate the phenomenon of hysteresis. Figure 1.4 depicts the line pinning of a droplet of liquid advancing on a surface that possesses discrete regions of differing surface energy relative to the liquid of the droplet. Hysteresis occurs due to the three-phase contact line being pinned by the lyophobic regions as the droplet advances as well as the line pinning that occurs at the lyophilic regions when the droplet is receding. ${ }^{9}$



Figure 1.4 Schematic illustrating the line pinning of a liquid droplet advancing on a chemically heterogeneous surface from a region of lower $\gamma$ to higher $\gamma$. 


\subsubsection{Influence of Surface Roughness on Mean Contact Angle}

For nominally rough surfaces the effect of surface roughness on the apparent contact angle is described by the Wenzel equation (Equation 1.4). In the Wenzel

$$
\cos \Theta_{\text {app }}=R_{\text {rough }} \cdot \cos \Theta
$$

equation the apparent contact angle $\left(\Theta_{\text {app }}\right)$, that is what would be observed by the eye, can be determined from the ratio between actual and projected surface area $\left(\mathrm{R}_{\text {rough }}\right)$ and actual contact angle $(\Theta)$. As $R_{\text {rough }}$ must be greater than or equal to one $\left(R_{\text {rough }} \geq 1\right)$ it follows that for non wetting surfaces $\left(\Theta \geq 90^{\circ}\right)$ the contact angle increases and for wetting surfaces $\left(\Theta \leq 90^{\circ}\right)$ the contact angle decreases as depicted by Figure 1.5. ${ }^{10}$

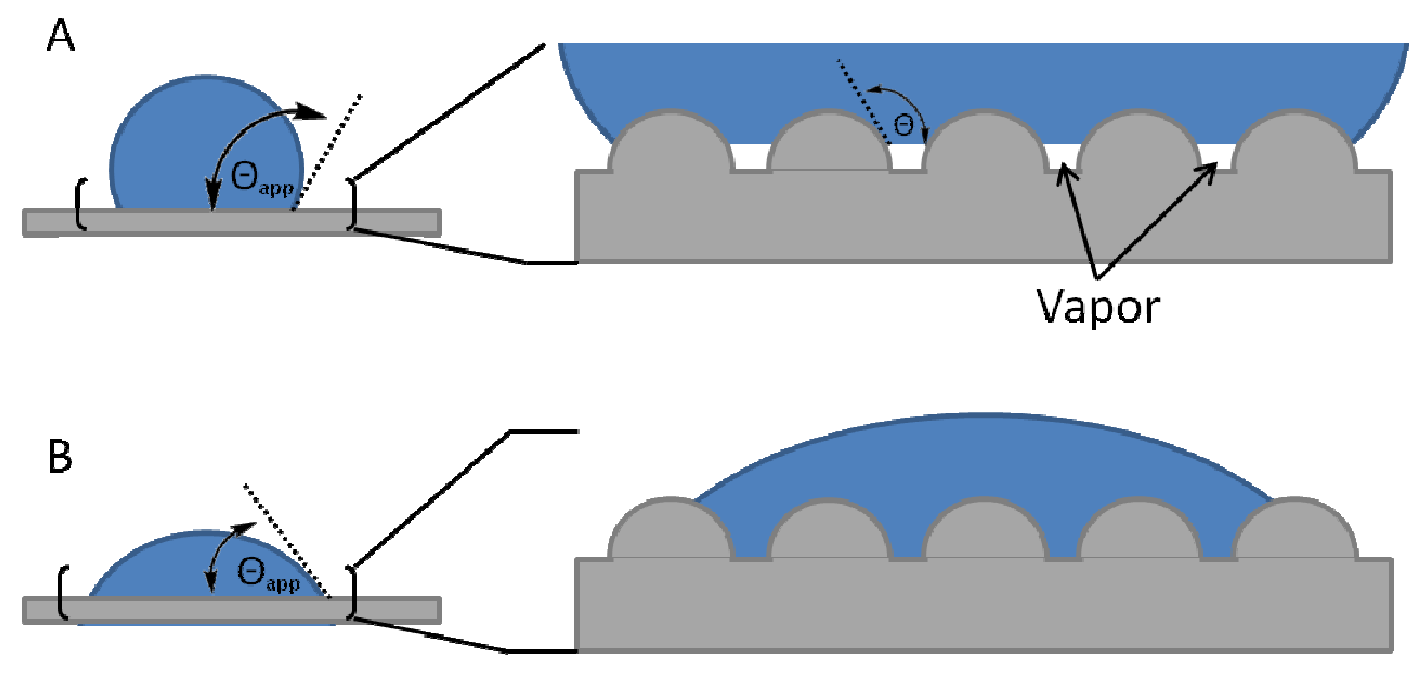

Figure 1.5 Schematic depiction of contact angle amplification of a nonwetting surface (A) and a wetting surface (B) due to surface roughness in accordance with the Wenzel equation. 


\subsection{Colloids}

A colloid is a disperse system in which one phase that exists in dimensions on the $\mathrm{nm}$ to $\mu \mathrm{m}$ scale is dispersed throughout a matrix of another phase. ${ }^{11}$ Table 1.1 displays the different types of colloidal systems. There is an intimate marriage of the chemistries interfaces and colloids as colloidal systems possess an incredible specific surface area that entirely dictates their behavior due to the enormity of the effect of surface properties. For colloidal systems consisting of dispersed solid particles the chemical modification of the surface of dispersed particles allows for the production of systems with tunable mechanical, catalytic, optical, magnetic and electrical capabilities.

Table 1.1 Types of Colloidal Systems

\begin{tabular}{ccc}
\hline Dispersed Phase & Matrix Phase & Name \\
\hline Gas & Solid & Solid Foam \\
Liquid & Solid & Solid Emulsion \\
Solid & Solid & Solid Suspension \\
Gas & Liquid & Foam \\
Liquid & Liquid & Emulsion \\
Solid & Liquid & Sol \\
Liquid & Gas & Liquid Aerosol \\
Solid & Gas & Solid Aerosol \\
\hline
\end{tabular}

Colloidal particles with at least one dimension having a size on between $1 \mathrm{~nm}$ and $100 \mathrm{~nm}$ are commonly referred to as nanoparticles. ${ }^{12}$ Nanoparticles frequently demonstrate behaviors that are intermediate between bulk solids and atomic or molecular systems. ${ }^{13}$ This can be illustrated by considering a particle composed of an inorganic crystal structure made up of a very small number of atoms. The properties that the particle exhibits cannot be that of a singular atom but will obviously differ from that of a macroscopic bulk material. ${ }^{14}$ 
The composition of nanoparticles spans a range of great chemical diversity, some of which includes metals, metal oxides, semiconductors, polymers, silicates, ceramics, organics, biomolecules and carbon allotropes. Nanoparticles can exist in different morphologies, most commonly as spheres, cylinders, disks, and tubes. ${ }^{12}$ Figure 1.6 illustrates the numerous features that can be tuned with nanoparticles. This diverse set of material composition and structure has led to numerous synthetic strategies based on gas, liquid and solid phase routes being developed for the creation of the different types of nanoparticles.

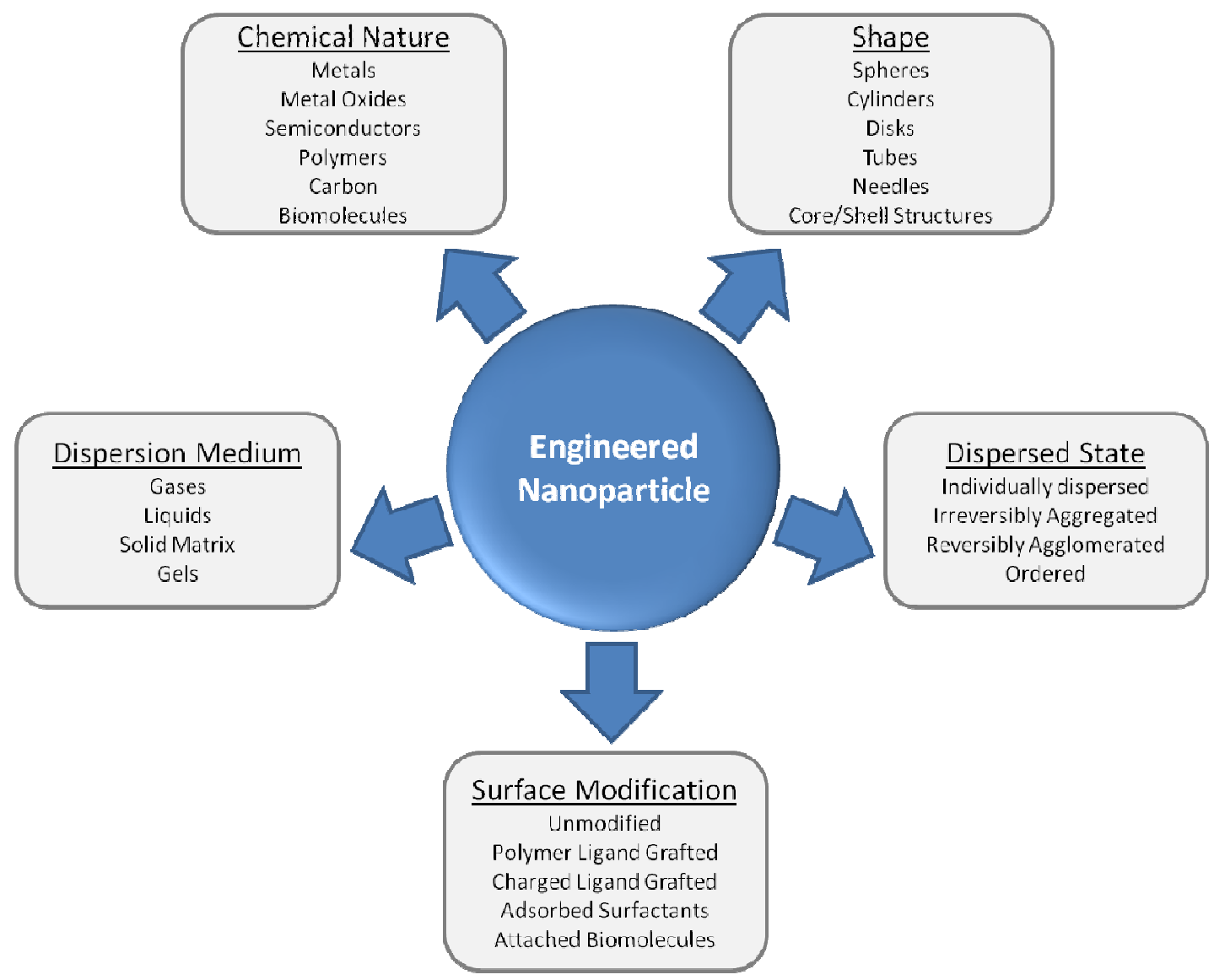

Figure 1.6 Tunable features of a nanoparticle. 
Surface modification of nanoparticles is commonly implemented for a number of reasons. One reason is to pacify a very reactive nanoparticle. Another purpose nanoparticle surface modification is to stabilize particles that have a propensity for aggregation in the medium in which they are dispersed. Also it is common to modify the surface of nanoparticles in order to introduce a desired set of chemical functionality. Finally it is also common to modify the surface in a fashion that can promote the assembly of the nanoparticles.

\subsection{Statement of Purpose}

The present study is undertaken to investigate various chemistries capable of both the creation and modification of surfaces with tunable surface energies, functionalities and catalytic activity. There are various end goal applications that have inspired the creation of these functional surfaces. The first is the creation of a vascular network for inclusion in self-healing materials. The function of this vascular system is to selfexpunge healing fluid in the event that the material in which it is contained is damaged. In order to develop a mechanism by which the system can self-expunge a fluid it is necessary to create a surface that had the ability to switch its surface energy in a patterned fashion via an external stimulus; the mechanism of this switch is facilitated by DielsAlder chemistry. Ultimately this will lead to the creation of a vascular system with "molecular gates" in the form of lyophilic and lyophobic regions that effectively control fluid flow without the need for mechanical pumps and gates.

The second goal of this study is the creation of an inorganic transparent anode that has a surface functionalized with a transparent conductive polymer brush. This system 
will be utilized in organic photovoltaic devices with an improved efficiency over current technologies that have heterogeneous composition and exhibit uneven conductivity. The creation of this system entails the modification of an indium tin oxide surface with polymerization initiation sites capable of facilitating a controlled radical surface initiated polymerization for the creation of well defined, extended polymer chains from the surface. The polymerization is undertaken utilizing a novel styrenic monomer that possesses pendant groups capable of creating conductive polymer chains via electropolymerization. The successful creation of aligned conductive polymer chains along a styrenic backbone grafted from a transparent provides a route towards a much more ideal conductive polymer layer to function as a contact for the transparent anode in organic photovoltaic devices.

Finally a nanoheterostructure composed of a CdSe seed, CdS rod, and Pt tip with the theoretical potential to facilitate photocatalytic hydrogren production is created. The unique electronic properties that are exhibited in materials at the nanoscale are utilized to facilitate the charge separation of photoinduced charge carriers necessary to activate catalytic hydrogen production at the surface of the Pt tip.

\subsection{References}

(1) Shaw, D. J. Introduction to Colloid ad Surface Chemistry; 4th ed.; Elsevier Science: Burlington, MA, 1992.

(2) Butt, H.-J.; Graf, K.; Kappl, M. Physics and Chemistry of Interfaces; WILEYVCH: Weinheim, Germany, 2003.

(3) Fawcett, W. R. Liquids, Solutions and Interfaces: From Classical Macroscpoic Descriptions to Modern Microscopic Details; Oxford University Press: New York, NY, 2004.

(4) Gao, L.; McCarthy, T. J. Langmuir 2009, 25, 14105-14115.

(5) Young, T. Philosophical Transactions of the Royal Society of London 1805, 95, 65-87. 
(6) Butt, H.-J.; Kappl, M. Surface and Interfacial Forces; Wiley-VCH: Weinheim Germany, 2010.

(7) Eick, J. D.; Good, R. J.; Neumann, A. W. Journal of Colloid Interface Science 1975, 53, 235.

(8) Huh, C.; Mason, S. G. Journal of Colloid Interface Science 1977, 60, 11.

(9) Swain, P. S.; Lipowsky, R. Langmuir 1998, 14, 6772.

(10) Quere, D. Physica A 2002, 313, 32.

(11) Shchukin, E. D.; Pertsov, A. V.; Amelina, E. A.; Zelenev, A. S. Colloid and Surface Chemistry; Elsevier Science: Amsterdam, The Netherlands, 2001.

(12) Nagarajan, R.; Hatton, T. A. American Chemical Society Symposium Series 2008.

(13) Nanoparticles From Theory to Application; Schmid, G., Ed.; Wiley-VCH: Weinheim Germany, 2004.

(14) Handbook of Nanophysics: Nanoparticles and Quantum Dots; Sattler, K. D., Ed.; CRC Press: Boca Raton FL, 2011. 


\section{Controlling Surface Energy and Wetability with Diels-Alder Chemistry}

\subsection{Introduction}

\subsubsection{Motivation and Brief Review of Previous Works}

A number of researchers have recently demonstrated the ability to create vascularized structures, using techniques such as hollow glass capillaries ${ }^{1-3}$ and directwrite fugitive inks. ${ }^{4,5}$ These vascularized systems could impart many new functionalities to structural materials, such as active thermal management, self-healing, and mechanical property adaptation. In a related field, rapid progress in microfluidic devices has led to important new capabilities in a range of applications, including forensics, genomics, pharmaceuticals, healthcare, and biodetection..$^{6,7}$

All of these applications require fluid flow in small channels. The driving force for this flow is typically provided by an external mechanical pump coupled to the flow system. However, for some applications, the weight, volume, complexity, and power requirements of conventional pumps are not acceptable, and techniques for developing internal pressurization without external devices is desired. Examples of such novel stand-alone systems would include a self-healing structural component in an aircraft, or a compact, low-cost biodetection device for remote military applications. Similarly, active control of internal flow characteristics such as path and velocity is typically executed via miniature valves and gates, which introduce system complexity while reducing robustness.

A less-burdensome alternative to mechanical pumps, valves, and gates is to control flow via surface effects. A channel wall that can be switched from wettable to non-wettable relative to the vascular fluid can be used to induce surface tension-driven 
pressurization of the flow. Patterning wettable and non-wettable surfaces enables controlled pressure drops, gated flow regions, and control of flow pathways. These approaches are analogous to the strategies used by plants to control vascular flow, ${ }^{8}$ and can be contrasted to the utilization of a heart (a centralized pump) in animals.

The concept of tailoring internal surface chemistry to control channel flows has been demonstrated previously. Liang et al. demonstrated a thermally-active glass capillary coating whose contact angle could be tailored from strongly hydrophilic to weakly hydrophilic by varying the temperature over a $30{ }^{\circ} \mathrm{C}$ temperature range. ${ }^{9}$ Suk and Cho showed that patterning hydrophobic and hydrophilic surfaces could be used to tailor microflow characteristics. ${ }^{10}$ Caprioli et al. demonstrated a photoactive capillary coating through which contact angle could be varied by up to $20^{\circ}$ via ultraviolet illumination. ${ }^{11}$

\subsubsection{Theoretical Modeling}

Consider the system shown in Figure 2.1. A cylindrical capillary of diameter D and length $L$ is filled with a fluid of viscosity $\mu$ with surface tension $\gamma$. The fluid-wall contact angle is $\theta$. At time $t=0$, the fluid-air interface is located at position $x=0$. The capillary is assumed to be horizontal, such that gravity effects can be neglected.

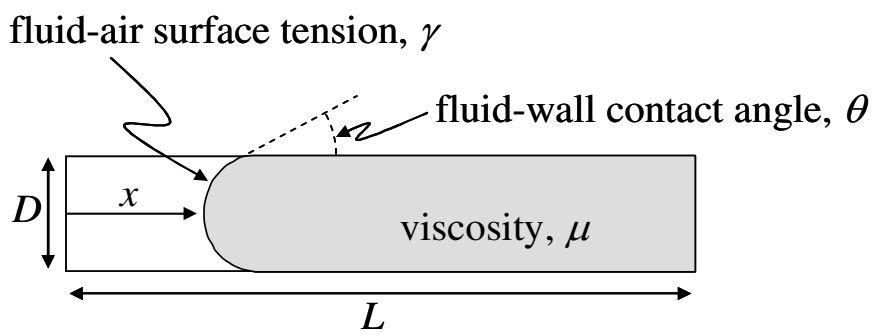

Figure 2.1 Schematic of a horizontally inclined capillary that defines problem. 
The capillary force $\mathrm{Fc}$ acting at the circumferential fluid-wall-air interface is given by Equation 2.1 where $\mathrm{Fc}>0$ indicates a force in the positive $\mathrm{x}$ direction. The pressure due to the interfacial tension is given by Equation 2.2.

$$
\begin{gathered}
F_{c}=(\pi D) \gamma \cos \theta \\
p=\frac{4 F_{c}}{\pi D^{2}}=\frac{4 \gamma \cos \theta}{D}
\end{gathered}
$$

Assuming the fluid empties into an open space at position $\mathrm{x}=\mathrm{L}$ with $\mathrm{p}=0$, then the length of capillary filled with fluid at any time $t>0$ is equal to $L-x$. Therefore, the pressure gradient associated with interfacial tension at any instant of time is given by Equation 2.3.

$$
\left.\frac{d p}{d x}(t)\right|_{\text {int.tension }}=\frac{4 \gamma \cos \theta}{D(L-x(t))}
$$

For $\mathrm{t}>0$, the fluid interface position $\mathrm{x}>0$ moves at velocity $\mathrm{v}(\mathrm{t})=\mathrm{dx} / \mathrm{dt}$. The viscous pressure drop associated with this flow is given by Equation 2.4, where $\mathrm{Q}(\mathrm{t})$ is defined by Equation 2.5 so that Equation 2.6 holds true.

$$
\begin{gathered}
\left.\frac{d p}{d x}(t)\right|_{\text {viscous }}=\frac{128 \mu}{\pi D^{4}} Q(t) \\
Q(t)=\frac{1}{4} v(t) \pi D^{2}
\end{gathered}
$$




$$
\frac{d p}{d x}(t)_{\text {viscous }}=\frac{32 \mu v(t)}{D^{2}}
$$

Equating the driving interfacial tension pressure gradient to the resisting viscous pressure drop yields Equation 2.7 which can be redefined as Equation 2.8. Equation 2.9 defines $y(t)$ and results in Equation 2.10, where $\alpha$ is defined by Equation 2.11.

$$
\begin{gathered}
\frac{32 \mu}{D^{2}} v(t)=\frac{4 \gamma \cos \theta}{D(L-x(t))} \\
v(t)=\frac{D \gamma \cos \theta}{8 \mu(L-x(t))} \\
y(t)=\frac{L-x(t)}{L} \\
\frac{d y}{d t}(t)=-\frac{1}{2 \alpha y(t)} \\
\alpha=\frac{4 \mu L^{2}}{D \gamma \cos \theta}
\end{gathered}
$$

Solving Equation 2.10 yields Equation 2.12, which can be redefined as Equation 2.13. Applying the initial condition $\mathrm{x}(\mathrm{t})=0$ at $\mathrm{t}=0$ gives $\mathrm{C} 1=1$, yields Equation 2.14, where $\alpha$ is a characteristic purge time, equal to the time required for the capillary to empty.

$$
y(t)=\sqrt{C_{1}-\frac{t}{\alpha}}
$$




$$
\begin{gathered}
\frac{x(t)}{L}=1-\sqrt{C_{1}-\frac{t}{\alpha}} \\
\frac{x(t)}{L}=1-\sqrt{1-\frac{t}{\alpha}}
\end{gathered}
$$

The instantaneous interface velocity can be defined by Equation 2.5, where (L / 2 $\alpha$ ) is the initial interface velocity. The interface velocity increases with time and position, approaching infinite velocity at the capillary exit (when there is no viscous force balancing capillary forces). These terms can also be expressed in terms of the capillary aspect ratio $\lambda=\mathrm{L} / \mathrm{D}$, Equation 2.16.

$$
\begin{gathered}
v(t)=\frac{L}{2 \alpha}\left(1-\frac{t}{\alpha}\right)^{-\frac{1}{2}} \\
\alpha=\frac{4 \mu D}{\gamma \cos \theta} \lambda^{2}
\end{gathered}
$$

\subsubsection{Experimental Strategy}

In the present study, Diels-Alder chemistries are combined with a range of functional groups to develop and demonstrate tailorable surfaces. In a traditional DielsAlder reaction, a diene is attacked by a dieneophile resulting in a ring structure. ${ }^{12,13}$ as shown in Figure 2.2. Ring formation is temperature dependent and reversible by controlling the 

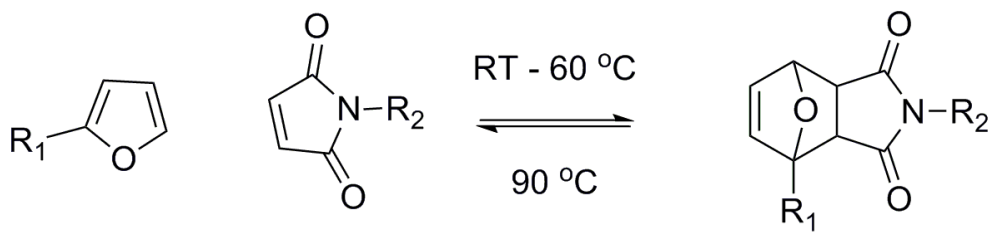

Figure 2.2 Reversible Diels-Alder adduct whose formation is temperature dependent.

heating and cooling of the system. Incorporating these reversible bonds on a surface should allow for the control of functional surface chemistries and could lead to surfaces exhibiting invertible surface energies. This technique has been applied to a variety of materials including the preparation of organic-inorganic polymer hybrids, ${ }^{14-17}$

thermoplastic elastomers, ${ }^{18,19}$ polyurethanes and foams. ${ }^{20}$ By incorporating these reversible bonds into functional surface chemistries, the ability to create tailorable surfaces that can be transitioned between hydrophobic and hydrophilic states is demonstrated. Initial screening studies characterized the surface characteristics of coatings on flat glass substrates. The best methodology was then applied to glass capillaries where the wetability and capillary fill properties were investigated.

\subsection{Results and Discussion}

\subsubsection{Flat Surface Modification and Subsequent Characterization}

Scheme 2.1 illustrates the synthetic methodology for the incorporation of DielsAlder linkages upon glass surfaces. Glass slides were first silanized with 3aminopropyltrimethoxysilane (APS) to yield amino-terminated surfaces (Surface 1). Amidation was then completed with 2-furfuryl chloride to prepare furan terminated 
surfaces (Surface 2), which upon introduction of compound 2, underwent a Diels-Alder reaction to yield a hydrophobic surface (Surface 3).

Scheme 2.1. Synthetic route for preparation of Diels-Alder functionalized glass slides. ${ }^{\mathrm{A}}$

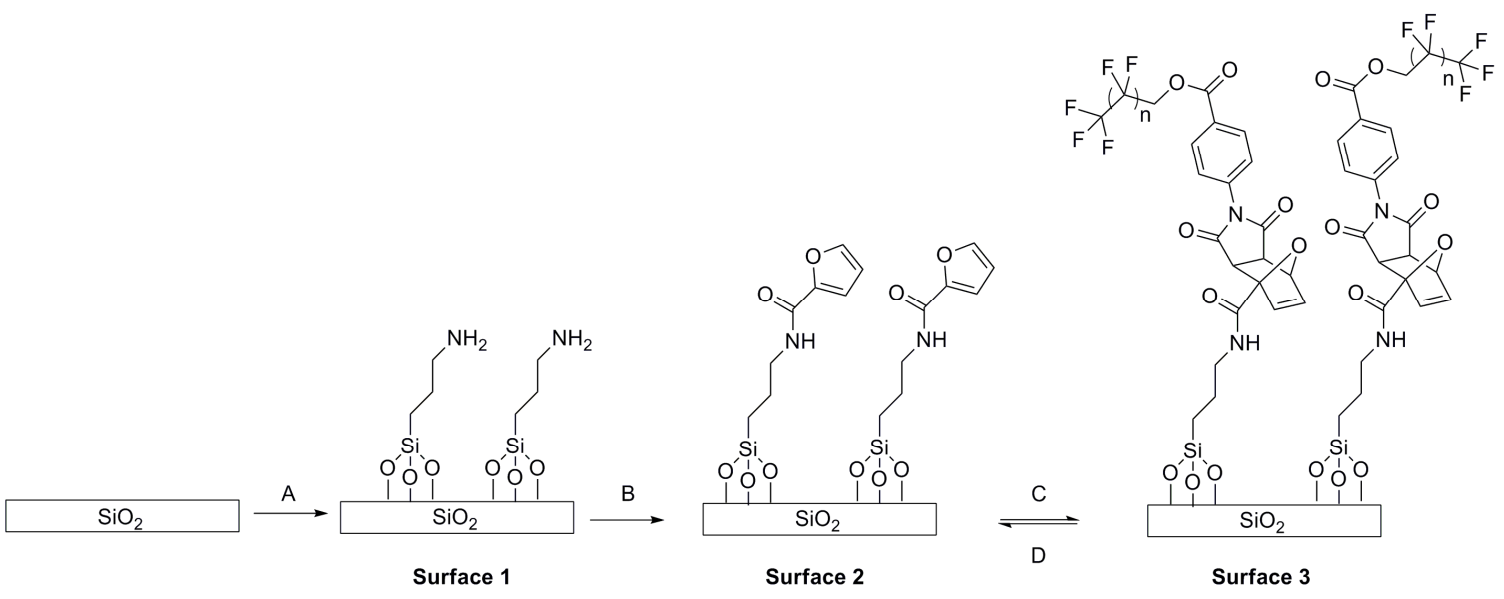

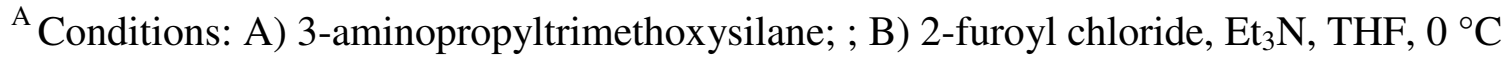
to RT, 24 h; C) Compound 4, THF, RT, 24 h.; D) Toluene, Reflux, 24 h.

Scheme 2.2. Synthetic route for preparation of fluorinated maleimide. ${ }^{\mathrm{A}}$

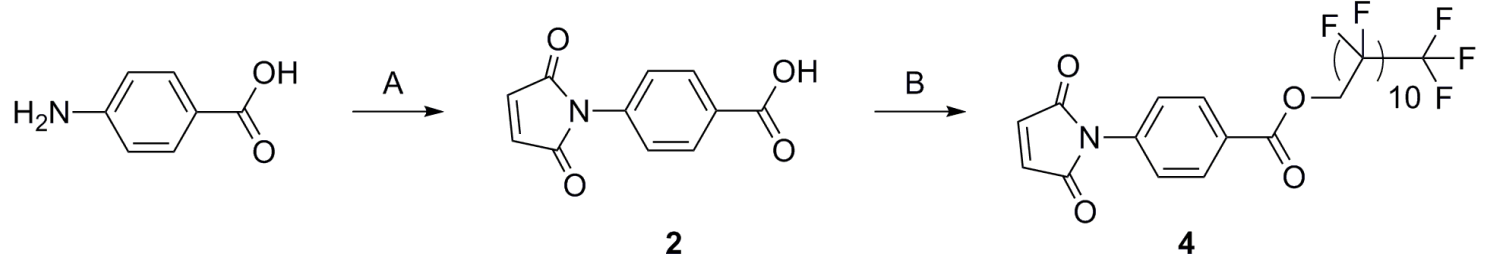

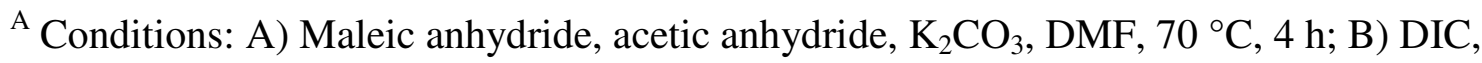
1H,1H-perfluoro-1-dodecanol, DMAP, THF, $55^{\circ} \mathrm{C}, 24 \mathrm{~h}$.

Compound $\mathbf{4}$ was prepared in a straight-forward manner by preparing 4carboxylate maleimide followed by carbodiimide coupling with 1H,1H-perfluoro-1- 
dodecanol as shown in Scheme 2. XPS analysis was utilized to confirm surface functionalization. Figure 2.3 displays a large emission corresponding to the presence of fluorine indicating that the Diels-Alder reaction was successful. Contact angle measurements were utilized to monitor the change in surface functionality and energy.

As displayed in Table 2.1, untreated glass slides were very hydrophilic (ca $\sim 23 \pm$ $3^{\circ}$ ). Silanization of the slides was effective as the contact angle displayed a significant hydrophobic shift due to the incorporation of an organic alkyl chain while maintaining a narrow standard deviation (ca $\sim 58 \pm 3^{\circ}$ ). A similar hydrophobic shift was observed upon

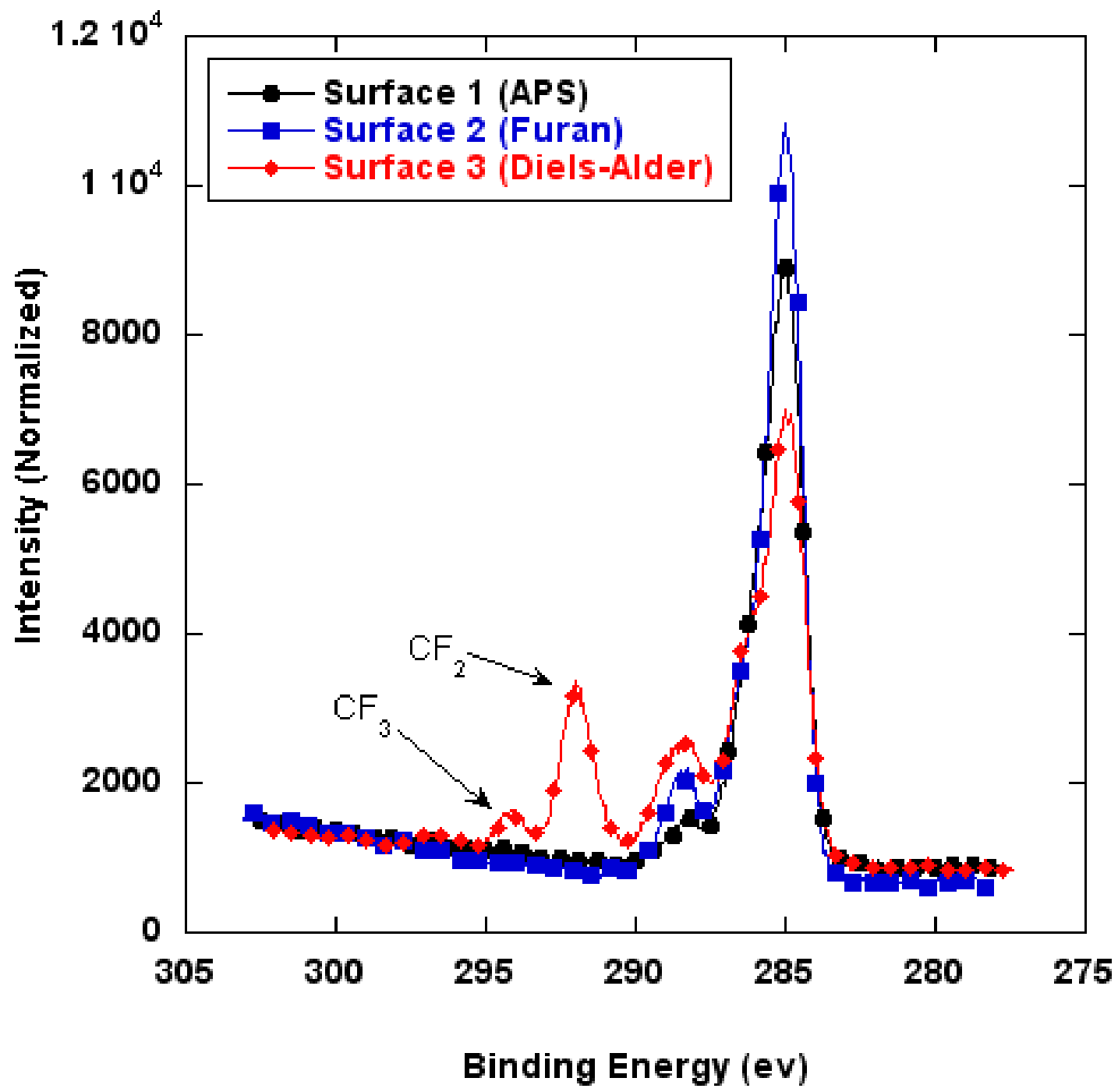


Figure 2.3. XPS analysis of functionalized surfaces corresponding to those listed in Table 1.

Table 2.1 Contact Angle Measurements of Glass Slides and Capillaries at Various States of Functionalization

\begin{tabular}{cccccc}
\hline & & \multicolumn{4}{c}{ Contact Angle } \\
\cline { 3 - 5 } Surface Functionality & $\begin{array}{c}\text { Corresponding } \\
\text { Capillary }\end{array}$ & $\begin{array}{c}\text { Fluid } \\
\text { Column }\end{array}$ & Static & Advancing & \multirow{2}{*}{ Receding } \\
\hline Untreated & Capillary A & $55^{\circ}$ & $23 \pm 3^{\circ}$ & $25 \pm 3^{\circ}$ \\
APS (Surface 1) & Capillary B & $80^{\circ}$ & $58 \pm 3^{\circ}$ & $60 \pm 3^{\circ}$ & $25 \pm 3^{\circ}$ \\
Furan (Surface 2) & Capillary C & $78^{\circ}$ & $70 \pm 3^{\circ}$ & $72 \pm 3^{\circ}$ & $50 \pm 3^{\circ}$ \\
Fluorinated (Surface 3) & Capillary D & $105^{\circ}$ & $101 \pm 9^{\circ}$ & $104 \pm 9^{\circ}$ & $33 \pm 12^{\circ}$ \\
Cleaved (Surface 2) & Capillary E & $81^{\circ}$ & $70 \pm 6^{\circ}$ & $70 \pm 6^{\circ}$ & $51 \pm 3^{\circ}$ \\
\hline
\end{tabular}

addition of the furan to the substrate $\left(\mathrm{ca} \sim 70 \pm 3^{\circ}\right)$. After completion of the Diels-Alder reaction with compound $\mathbf{4}$ a substantial change in contact angle was observed as the surface became fully hydrophobic $\left(\mathrm{CA} \sim 101 \pm 9^{\circ}\right)$. The fluorinated alkyl chain reduced the surface energy which increased the contact angle, although a larger standard deviation was observed. The scatter in the data was most likely caused by surface heterogeneity caused by the formation of crystalline domains, as it is well established that perfluorinated alkyl chains greater than eight carbons in length will crystallize. ${ }^{21}$ To probe this assertion, a six carbon fluorinated alkyl chain was employed to prepare a shortchain analog (compound 3) that would be unable to crystallize due to the shorter chain length. In this sample, the contact angle was $65 \pm 2^{\circ}$, which was lower than the initial furan surface. It is hypothesized that the Diels-Alder linkage adopted a conformation in which the hydrophobic tail was intercalated within the organic layer which exposed either the amide or ester linkages within compound 3. Treatment of the slides within refluxing toluene induced the retro Diels-Alder reaction and removed the hydrophobic chain end, 
as indicated by the decrease in contact angle to approximately the same value as that obtained for original furan terminated state $\left(\mathrm{CA} \sim 70 \pm 6^{\circ}\right)$. Figure 2.4 displays an optical
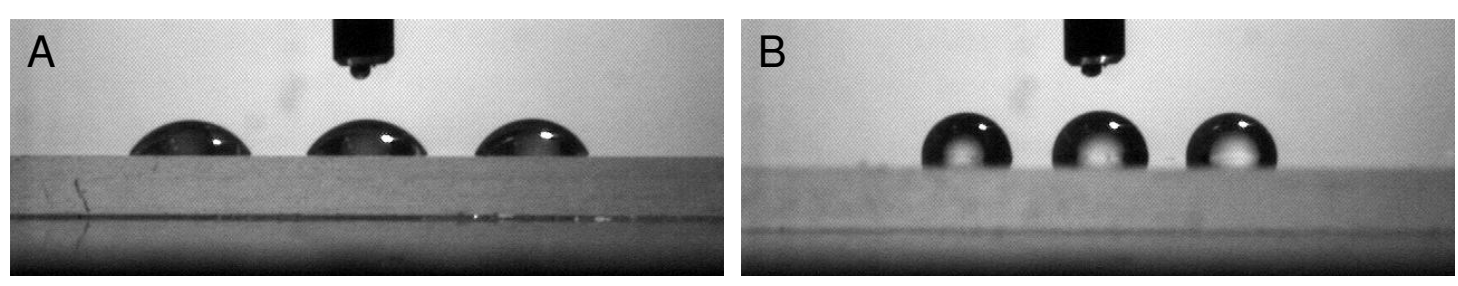

Figure 2.4 Optical micrograph of contact angle measurements of (A) furan functionalized slide (Surface 2) and (B) fluorinated functionalized slide (Surface 3).

micrograph of Surfaces 2 and 3, demonstrating a significant change in the wetability of the surface.

\subsubsection{Diels-Alder System Optimization}

Several attempts were made to explore the versatility of the system in order to optimize the change in surface energy between the hydrophilic and hydrophobic states. Ideally, the contact angle difference between the hydrophilic and hydrophobic states should be maximized to provide the most efficient driving of fluid from a capillary. Several different diene and dieneophile combinations were explored utilizing the compounds depicted in Figure 2.5. However, only the C4 combination displayed an appropriate change in contact angle. Combinations B1, B2, C1 and C3 did not display a large enough change in the surface energy. For example, surfaces functionalized with compound $\mathrm{B}$ and $\mathrm{C}$ displayed an initial contact angle of $66 \pm 6^{\circ}$ and $70 \pm 3^{\circ}$, respectively. Upon formation of the DA adduct, the contact angle changed to $63 \pm 4^{\circ}$ and $64 \pm 2^{\circ}$ for the $\mathrm{B} 1$ and $\mathrm{C} 1$ combination. Other synthetic challenges to consider are the electronics of 
the Diels-Alder reaction. Typically, the dieneophile must be electron deficient while the diene should be electron rich. For example, compound $\mathbf{2}$ was coupled to an amino

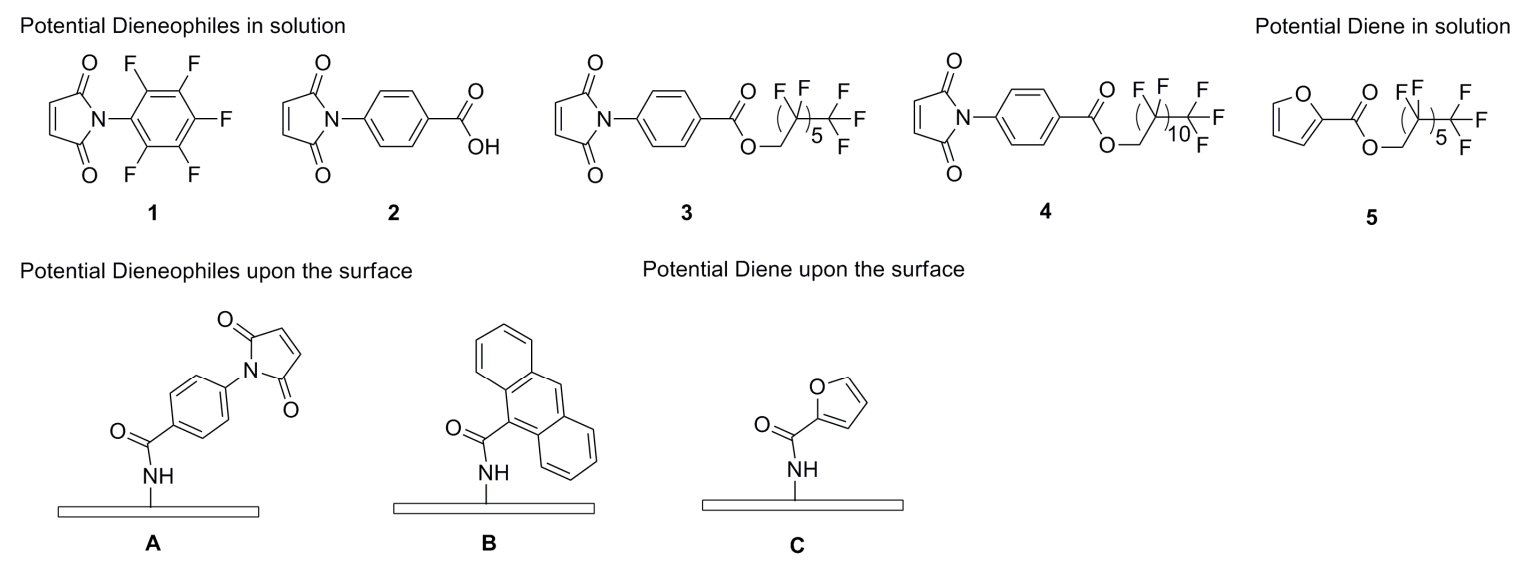

Figure 2.5 Various Diels-Alders systems that were utilized to prepare thermo-responsive surfaces.

terminated slide with a carbodiimide coupling agent to yield a maleimide terminated surface with a contact angle of approximately $65 \pm 2^{\circ}$, which is lower than the furan terminated surface. Next, compound 5 was prepared by coupling together $1 \mathrm{H}, 1 \mathrm{H}-$ perfluoro-1-dodecanol and 2-furfuryl chloride in the presence of $\mathrm{Et}_{3} \mathrm{~N}$. Unfortunately, upon introduction of the fluorinated diene, no Diels-Alder reaction occurred as the contact angle remained unchanged at approximately $65 \pm 2^{\circ}$. By coupling the fluorinated alcohol to the furan ring via an ester linkage, electron density was withdrawn from the diene creating an electron poor diene which inhibited the formation of the Diels-Alder linkage.

\subsubsection{Capillary Modification and Characterization}

Selecting the system depicted in Scheme 1 (Combination C4, dieneophile coupled to surface), the methodology was transitioned to glass capillaries. Following analogous 




Figure 2.6 Optical micrograph of capillaries at various stages of functionalization filled with $\mathrm{H}_{2} \mathrm{O}$ containing fluorescein taken under UV conditions. Letters refer to capillaries as denoted in Table 1. Numerical values represent the height of capillary fill. Each grid block represents $0.5 \mathrm{~cm}$. [Note - fluorescent $\mathrm{H}_{2} \mathrm{O}$ was utilized to aid the eye. It has no effect on capillary action.]

synthetic protocols, one mm internal diameter capillaries were prepared and characterized. As conventional contact angle measurements were not possible inside the capillaries, a series of flow experiments were conducted to observe the impact of surface functionality upon capillary fill. Preliminary work evaluated static capillary fill, where a capillary from each stage of functionalization was oriented vertically and partially immersed into a $\mathrm{H}_{2} \mathrm{O}$ reservoir and the height of capillary fill height relative to the reservoir height was recorded. Attempts to visually capture the capillary fill process were difficult due to scattering and low contrast. Therefore, fluorescein, a fluorescent dye, was dissolved in $\mathrm{H}_{2} \mathrm{O}$, and an optical micrograph was captured under UV conditions as shown 
in Figure 2.6. Table 1 shows the apparent contact angle for these glass capillaries, which can be estimated from the wicked fluid column height $h$ according to Equation 2.17.

$$
h=\frac{4 \gamma \cos \theta}{\rho g D}
$$

Where the fluid has density $\rho$ and surface tension $\gamma$,and exhibits a contact angle of $\theta$ relative to the capillary with internal diameter $D$. The results show that the behavior of the capillary closely follows that of the glass microscope slides, indicating that the treatment is equally applicable to external surfaces and internal flow channels.

\subsubsection{Patterned Hydrophilic/Hydrophobic Capillaries}

\subsubsection{Preparation of Patterned Hydrophilic/Hydrophobic Capillaries}

As observed with the untreated glass slide, the as-received capillary (A) exhibited hydrophilic properties as indicated by the significant capillary action. Capillaries B and C also displayed fluid advance, although Capillary D displayed little to no capillary action. The presence of the hydrophobic perfluorinated alkyl chains upon the surface prevented spontaneous fluid uptake of the capillary. After thermal treatment of Capillary D enabled the retro Diels-Alder reaction to occur, the hydrophobic maleimide was removed from the surface to yield Capillary E. The retro-Diels-Alder surface exhibited water uptake analogous to Capillary $\mathrm{C}$ as the capillary fill for these surfaces was identical within experimental error.

After successfully demonstrating the ability to control capillary fill by manipulating the surface functionality, patterned capillary tubes were prepared by immersing half of the capillary tube within refluxing toluene for $12 \mathrm{~h}$ to induce the retro- 
Diels-Alder reaction and yield a capillary tube that was hydrophilic along half of its length, and hydrophobic along the remainder of its length.

\subsubsection{Demonstration of Flow Gating}

To demonstrate the utility of a patterned capillary for flow gating, a fluid slug (dyed water) was introduced into the hydrophilic region of the capillary as shown in Figure 2.7A. The capillary was then rotated $90^{\circ}$ to a vertical orientation. Upon rotation, the fluid slug falls through the hydrophilic region, as the surface tension forces on either side of the slug exactly balance and the only flow resistance is viscous dissipation due to internal flow. ${ }^{22}$ When fluid slug reaches the hydrophobic zone, however, the advancing slug surface is in contact with a hydrophobic surface, while the trailing slug surface is in contact with a hydrophilic surface as shown in Figure 2.7B. The resulting contact forces on each surface work in tandem to resist gravity and, if the droplet weight is less than the contact forces (as in this experiment), the droplet is brought to rest. For more massive slugs or slugs driven by other constant external forces (such as electrically-driven flows), the slugs can be driven through this transitional zone but the velocity will drop relative to flow through the uniform capillary. Therefore, these types of patterned surfaces can be used to arrest or slow fluid slugs in channel flows.

\subsubsection{Flow Experiments}

To further probe the capillary forces within the patterned samples, a dynamic filling experiment was conducted. A schematic of the experimental setup is shown by Figure 2.8. To briefly describe the experiment, a large fluid reservoir $(\sim 2 \mathrm{~L})$ was placed upon a 


specimen $90^{\circ}$

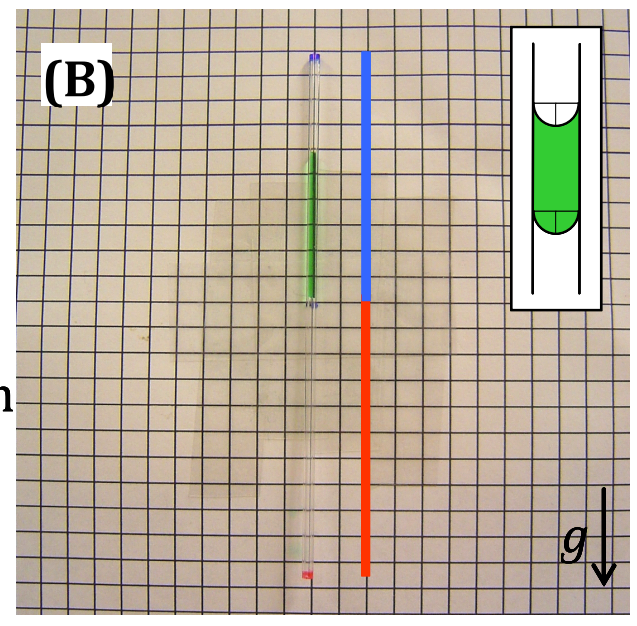

Figure 2.7 Optical micrograph of a patterned capillary containing an internal fluid slug (dyed $\mathrm{H}_{2} \mathrm{O}$ ). The red line segment indicates the hydrophobic length of the capillary, while the blue line segment indicates the hydrophilic region. Gravity is oriented downward in both images. Inset schematics represent slug interface characteristics for each case.

vertically adjustable stand to regulate the height of the reservoir. A patterned capillary was then connected to the fluid reservoir via a plastic tube. The bottom of the capillary and the top of the fluid level in the reservoir were set to the same height. The elevation of the reservoir was increased at two mm increments while the capillary maintained a constant position. Between each adjustment of the reservoir, the system was allowed to equilibrate for at least one minute. Measurements were made from the top of the fluid in the reservoir to the top of the fluid within the capillary. Raising the reservoir was equivalent to lowering the capillary within the fluid reservoir, but this configuration allowed for a precise measurement of fluid level within the tube. By utilizing the apparatus described by Figure 2.8 one would expect capillary filling to be observed as illustrated by the theoretical model shown in Figure 2.9. Optical micrographs of capillaries during the dynamic capillary fill experiment as displayed in Figure 2.10. 


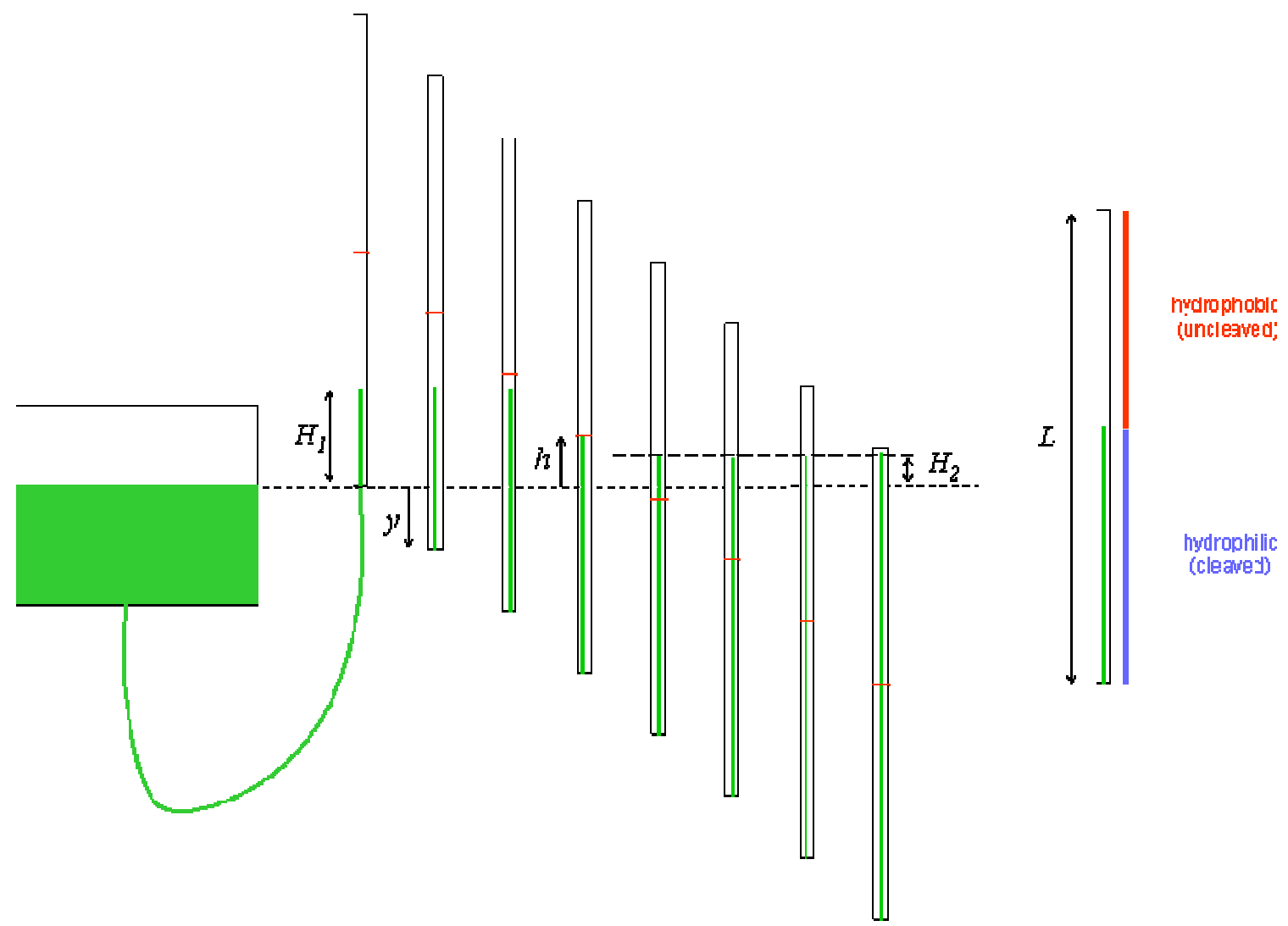

Figure 2.8 Schematic representation of the dynamic capillary filling experiment. actual experiment was conducted by raising the reservoir, which would have the same effect as lowering the capillary.

Figure 2.11 plots the distance between the top of the reservoir level and the height of fluid within the capillary which was found to be in excellent agreement with the theoretical model. Control experiments were conducted with non-patterned capillaries to observe a baseline, corresponding to the hydrophilic and hydrophobic listing in Figure 2.10. For the hydrophilic surface, a positive difference between the reservoir and capillary height was observed as the reservoir level was raised. This is in agreement with the static capillary measurements and demonstrates the natural capillary action for a hydrophilic capillary, whereas the hydrophobic surface exhibited a negative difference 


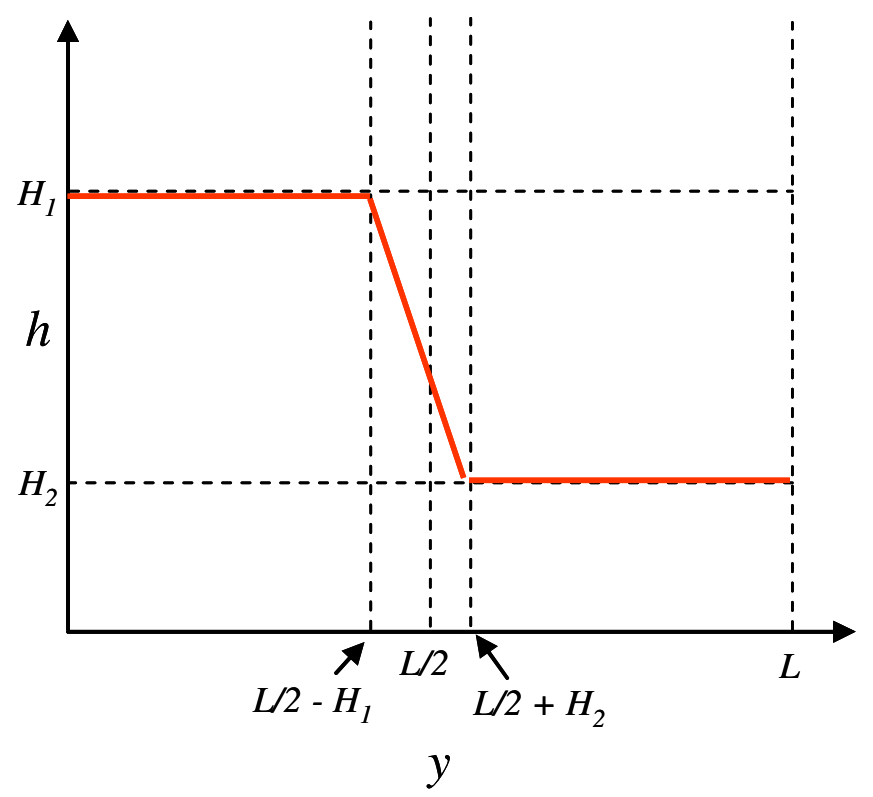

Figure 2.9 Ideal theoretical filling experiment of a patterned capillary, where $\mathrm{H} 1$ is the nominal vertical capillary wetting height for strongly hydrophilic capillary, $\mathrm{H} 2$ is the nominal vertical capillary wetting height for weakly hydrophobic capillary, $y$ is the vertical position of bottom of capillary relative to reservoir height, $h$ is the stable, instantaneous position of fluid column meniscus relative to reservoir height, and L is total capillary length.

between the capillary height and reservoir level. Again, this is in agreement with the static capillary measurements. The patterned capillary displays three distinct filling regions. Initially, the hydrophilic area of the patterned capillary was attached to the reservoir, which induced capillary action similar to the hydrophilic control experiment. This is denoted by $\mathrm{H}_{1}$, which corresponds to the capillary wetting height for strongly hydrophilic capillary.

The capillary action tracked with the control as the height of the reservoir was raised until the fluid level reached the hydrophobic region. At the interface, a second filling region was observed. The advance of fluid in the capillary was hindered by the hydrophobic surface, and a significant height differential was necessary to overcome the 
interface, corresponding to the Transition filling region. Once the reservoir reached a specific height, the pressure gradient was able to overcome surface tension forces, and the final filling region was reached. Capillary action then proceeded in a manner consistent with the hydrophobic control experiment, corresponding to $\mathrm{H}_{2}$, the capillary wetting height for a weakly hydrophobic capillary. Oscillation within the hydrophobic region is caused by the opposition of the surface tension within the capillary and back pressure created by height of the reservoir. This behavior was observed in both the hydrophobic capillaries and the hydrophobic regions of the patterned capillaries. After clearly demonstrating the ability to pattern capillary surfaces and control capillary action, efforts were made to achieve spontaneous purging of fluid from hydrophobic capillaries to enable the development of self-pressurizing tubules. For a fluid-filled capillary with one surface tension-driven interface and one stress-free interface, as shown in Figure 2.12, the characteristic purge time $\alpha$ is expressed, as previously derived in Equation 2.16. Where $\mu$ is fluid viscosity and $\lambda=L / D$ is capillary aspect ratio. The characteristic purge time can be used as a relative metric to compare the effects of fluid, surface, and geometric conditions on the absolute time scale for completely purging a capillary of its vascular fluid. Figure 2.12 shows characteristic purge time as a function of contact angle and capillary diameter, for a water-filled capillary with an aspect ratio of 100. Purge times are, in all cases shown, less than $10 \mathrm{~s}$ and, in most cases, less than $1 \mathrm{~s}$. This result shows that capillary-driven purging can be extremely rapid, down to ms for fine

$$
\alpha=\frac{4 \mu D}{\gamma \cos \theta} \lambda^{2}
$$

capillaries with strong non-wetting characteristics. Decreasing capillary diameter and 



Figure 2.10 Optical micrographs of dynamic capillary fill experiment. A) Hydrophilic capillary analogous to Surface 1 and Capillary C/E; B) Patterned capillary (bottom half hydrophilic, top half hydrophobic), C) Hydrophobic calillary analogous to Surface 2 and Capillary D.

increasing contact angle leads to more rapid purging. Note that increasing contact angle from $95^{\circ}$ to perfectly non-wetting $\left(180^{\circ}\right)$ results in a reduction in purge times or only one order-of-magnitude. This result indicates that even slightly non-wetting flow channels should provide efficient purging. 


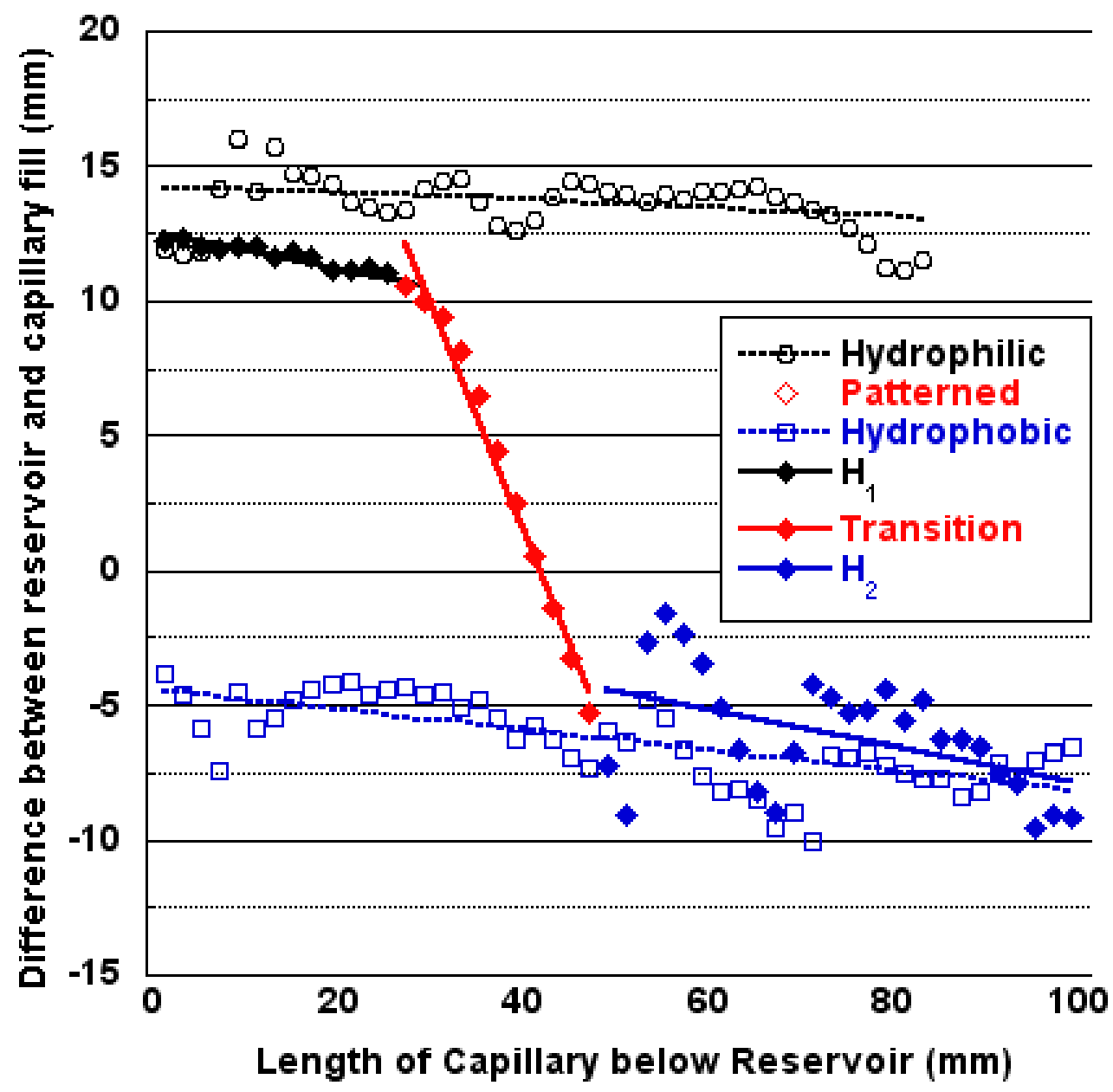

Figure 2.11 Dynamic capillary fill experiments comparing capillary action in reference to the height of the reservoir against the depth of the capillary.

Experiments were conducted to demonstrate this self-purging behavior, utilizing fluorinated capillaries (Surface 3) and dyed water. The experimental section contains schematics of experiments attempted. Unfortunately, self-purging was not observed experimentally. The limiting factor is the hysteretic nature of the functionalized surface. (Table 1) The fluorinated surfaces exhibit hydrophobic character during advancing flow (contact angle of $104^{\circ}$ ), but regress to a highly wetting surface during receding flow (contact angle of $33^{\circ}$ ). Therefore, once fluid is introduced into the capillary, the surface 

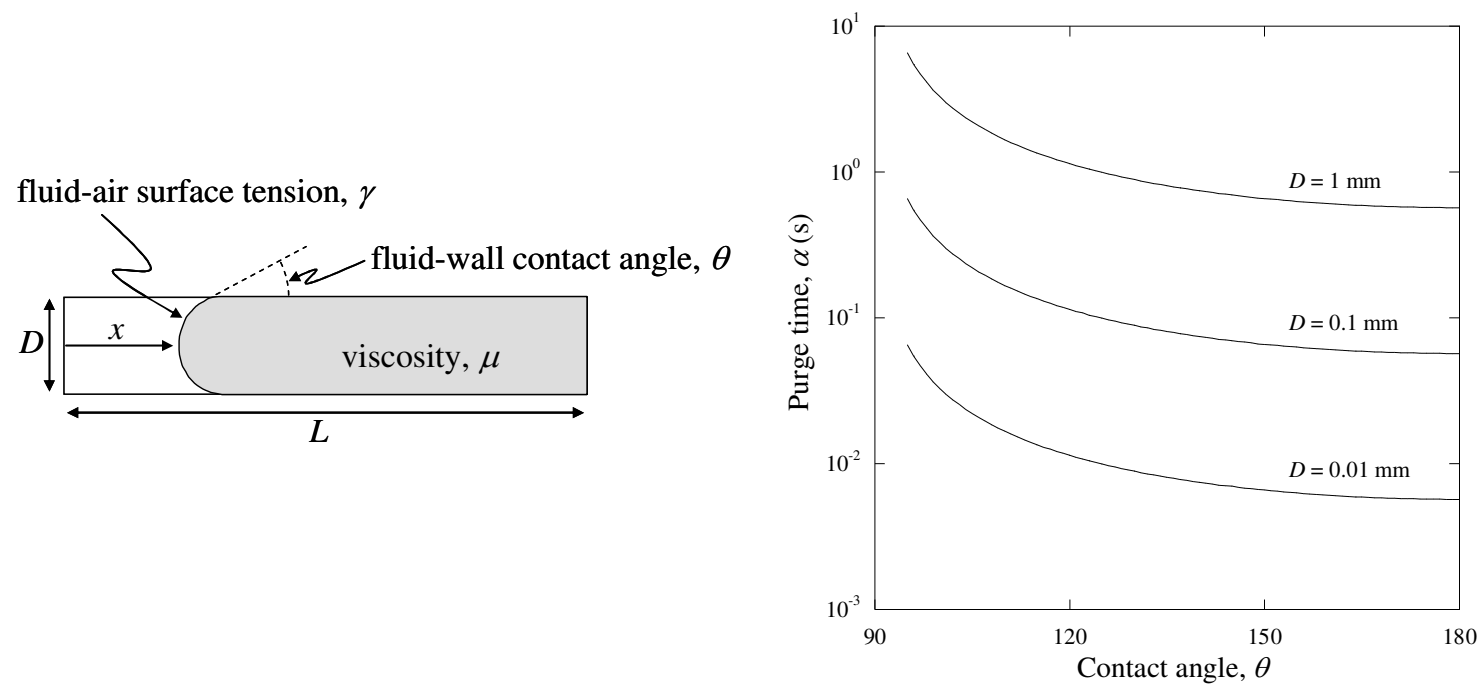

Figure 2.12 Left: Schematic of purge model; Right: Resulting purge time as a function of contact angle and diameter, for a water-filled capillary with an aspect ratio of 100 .

energy is altered and there is no longer a driving force to purge the fluid. It is well established that functionalizing glass slides with silanizing agents creates a porous and non-uniform coating. ${ }^{23,24}$ Such a treatment yields a surface with many functional groups; however, not all may be accessible due to steric hinderance. It is therefore hypothesized that residual amine functional groups are still present due to incomplete functionalized. These functional groups are able to $\mathrm{H}$-bond to $\mathrm{H}_{2} \mathrm{O}$ and create a residual hydrophilic surface upon exposure to $\mathrm{H}_{2} \mathrm{O}$. It may also be possible that surface rearrangements are occurring in which the amide, ester and ether bonds present are also able to H-bond to $\mathrm{H}_{2} \mathrm{O}$. Research is currently underway to develop new coating methodology to eliminate this possibility.

\subsection{Conclusions}


Utilizing Diels-Alder chemistry, thermo-responsive surfaces were prepared. The hydrophilicity of the surface was dictated by the functionalization of the surface. Hydrophobic dieneophiles were prepared and attached to glass slides and capillaries to yield a non-wetting surface. Thermal treatment of the surfaces cleaved the Diels-Alder linkage, and resulted in the fabrication of a hydrophilic surface. Preliminary analysis utilized contact angle (CA) measurements to monitor the change in surface energy, and observed a hydrophilic state $\left(\mathrm{CA}-70 \pm 3^{\circ}\right)$ before attachment of the dieneophile to a hydrophobic state $\left(\mathrm{CA}-101 \pm 9^{\circ}\right)$ followed by regeneration of the hydrophilic state $(\mathrm{CA}$ $70 \pm 6^{\circ}$ ) upon cleavage of the Diels-Alder linkage. The technology was then transferred to glass capillaries to control capillary fill, which was found to be dependent upon the surface functionality. Patterned capillaries were prepared and effective in serving as gates to inhibit fluid flow. Attempts to demonstrate a self-purging capillary were unsuccessful due to incomplete surface functionalization or surface rearrangement. Residual functional groups such as amines, amides, esters, and/or ethers were most likely involved in Hbonding which resulted in a residual $\mathrm{H}_{2} \mathrm{O}$ layer that would not support a purging phenomenon.

\subsection{Experimental}

\subsubsection{Materials}

All materials were purchased from commercially available sources and used without further purification.

\subsubsection{Instrumentation}


${ }^{1} \mathrm{H}$ and ${ }^{13} \mathrm{C}$ NMR was conducted on either a $400 \mathrm{MHz}$ Bruker or $600 \mathrm{MHz}$ Varian spectrometer in $\mathrm{CDCl}_{3}$, chemical shifts are reported as $\delta(\mathrm{ppm})$, referenced to the residual solvent peak. Contact angles were measured using a goniometer equipped with a digital camera. XPS analysis was completed at the Materials Research Laboratory at the University of California, Santa Barbara. High resolution carbon scans were collected at a $0^{\circ}$ detector angle. The images were recorded and processed with LabView software. Nanopure water was applied in approximately $2 \mu \mathrm{L}$ droplets. The circle about the droplet was defined and the tangent angle with the surface determined. Capillary surface energy was characterized by immersing the end of a treated capillary into $1.5 \mathrm{~cm}$ of dyed water. The distance of water uptake was measured and recorded.

\subsubsection{Synthetic Preparations}

2.4.3.1 Synthesis of 4-(2,5-dioxo-2,5-dihydro-1H-pyrrol-1-yl)benzoic acid (carboxy maleimide, compound 2). ${ }^{25}$

A $500 \mathrm{~mL}$ Erlenmeyer flask equipped with a magnetic stirbar was loaded with maleic anhydride (10 g, $102 \mathrm{mmol})$ and acetone $(150 \mathrm{~mL})$. The mixture was stirred until dissolved to which 4-aminobenzoic acid (13.98 g, $102 \mathrm{mmol})$ was slowly added. The mixture was stirred at room temperature for 2 hours during which a yellow precipitate was formed. The precipitate was collected via vacuum filtration, washed with acetone, dried and transferred to a $200 \mathrm{~mL}$ round bottom flask equipped with a magnetic stirbar. Potassium carbonate $(500 \mathrm{mg}, 3.6 \mathrm{mmol})$ was added to the flask followed by acetic anhydride (19.3 mL, $204 \mathrm{mmol})$ in dimethylformamide (DMF) $(75 \mathrm{~mL})$. The reaction mixture was stirred at $80^{\circ} \mathrm{C}$ for 2 hours and then allowed to come to room temperature. 
Subsequently the mixture was poured into an excess of ice water to obtain a pale yellow precipitate that was collected via vacuum filtration. $19.9 \mathrm{~g}$ yield $(90 \%) .{ }^{1} \mathrm{H}$ NMR: $\delta$ (ppm) $8.11(2 \mathrm{H}, \mathrm{d}), 7.62(2 \mathrm{H}, \mathrm{d}), 7.03(2 \mathrm{H}, \mathrm{s})$.

2.4.3.2 Synthesis of $\mathrm{N}-(1 \mathrm{H}, 1 \mathrm{H}-$ perfluoro-1-dodecyl phenyl carboxylate) maleimide (compound 4)

A $250 \mathrm{~mL}$ round bottom flask equipped with a magnetic stirbar was loaded with compound $2(5.00 \mathrm{~g}, 23.02 \mathrm{mmol})$ and tetrahydrofuran (THF) $(100 \mathrm{~mL})$. The mixture was stirred until dissolved to which diisopropylcarbodiimide (DIC) $(3.92 \mathrm{~mL}, 25.3 \mathrm{mmol})$ was added and the solution stirred for $15 \mathrm{~min}$. Next, 1H,1H-perfluoro-1-dodecanol (15.00 $\mathrm{g}, 25.00 \mathrm{mmol})$ and dimethylaminopyridine (DMAP) (146 mg, $1.20 \mathrm{mmol}$ ) were added to the solution and allowed to stir overnight at $55^{\circ} \mathrm{C}$. The solution was poured over methanol and the precipitate collected via vacuum filtration to yield compound 4 ( $>90 \%$ yield) as a dark red solid. ${ }^{1} \mathrm{H}$ NMR: $\delta(\mathrm{ppm}) 7.61(2 \mathrm{H}, \mathrm{d}), 7.42(2 \mathrm{H}, \mathrm{d}), 6.73(2 \mathrm{H}, \mathrm{s}), 4.82$ $(2 \mathrm{H}, \mathrm{s})$.

2.4.3.3 Synthesis of 1H,1H-perfluorohexylcyclopenta-1,3,-dienecarboxylate (compound 5).

A $200 \mathrm{~mL}$ round bottom flask equipped with a stirbar was loaded with dichloromethane (100 mL), 1H,1H-perfluror-1-hexanol (2.5 g, $9.16 \mathrm{mmol})$ and triethylamine $(1.39 \mathrm{~mL}, 9.96 \mathrm{mmol})$. The mixture was stirred until dissolved and then 
cooled to $0^{\circ} \mathrm{C}$ with an ice bath. 2-Furoyl chloride $(0.90 \mathrm{~mL}, 8.3 \mathrm{mmol})$ was added dropwise and the allowed to come to room temperature while stirring overnight. The solution was washed with deionized water $(2 \times 75 \mathrm{~mL})$, sat. $\mathrm{NaHCO}_{3}(2 \times 75 \mathrm{~mL})$ and again with dieonized water $(2 \times 75 \mathrm{~mL})$, dried over anhydrous magnesium sulfate and concentrated in vacuo to yield an orange oil.

\subsubsection{Typical Methodology for Surface Preparation}

An ethanol: $\mathrm{H}_{2} \mathrm{O}(90: 10)$ solution was acidified to $\mathrm{pH} \sim 5$ with glacial acetic acid. Next, (3-aminopropyl)trimethoxy silane (1\% wt/wt) was added and the mixture was allowed to stir for $5 \mathrm{~min}$. The solution was transferred to an appropriate container to which either glass slides or capillaries were added and allowed to incubate for 90 secs. Surfaces were then removed from the solution, rinsed with methanol, blown dry with nitrogen and set in an oven at $\sim 50{ }^{\circ} \mathrm{C}$ for 1 hour to yield amine terminated surfaces.

Next, an appropriate container was loaded with the surfaces (e.g capillaries or glass slides), THF and triethylamine $\left(\mathrm{Et}_{3} \mathrm{~N}, 0.030 \mathrm{M}\right)$. Next, 2-furoyl chloride $(0.025 \mathrm{M})$ was added and the surfaces were treated with the reaction mixture at room temperature overnight for 24 h. Next surfaces were rinsed with ethanol, blown dry with nitrogen and set in an oven at $\sim 50{ }^{\circ} \mathrm{C}$ for 1 hour to yield furan terminated surfaces.

Finally, an appropriate container was loaded with compound $4(0.03 \mathrm{M})$ and THF and agitated until homogenous. Next, surfaces were added to the solution and treated for $24 \mathrm{~h}$ at room temperature. After incubation, the surfaces were rinsed with ethanol, blown dry with nitrogen and set in an oven at $\sim 50{ }^{\circ} \mathrm{C}$ for 1 hour to yield DA terminated surfaces. 
Cleavage of the DA was completed by placing the surfaces in refluxing toluene for $24 \mathrm{~h}$, at which point the surfaces were rinsed with ethanol, blown dry with nitrogen and set in an oven at $\sim 50{ }^{\circ} \mathrm{C}$ for 1 hour to re-isolate the furan terminated surfaces.

\subsubsection{Capillary Experiments}

\subsubsection{Static Fill}

A typical static capillary fill experiment was conducted by partially immersing a capillary within a fluid reservoir to a depth of $1 \mathrm{~cm}$. To improve visual clarity, glass plates were placed adjacent to the capillary array between the array and the camera. The system was permitted to equilibrate for at least ten minutes prior to recording fluid column heights. For fluid slug experiments, a fluid slug of approximately $30 \mathrm{~mm}$ was drawn into the hydrophilic end of a partially-treated capillary via suction. The capillary was oriented horizontally and photographed, and then rotated $90^{\circ}$ to a vertical position and allowed to equilibrate prior to photographing.

\subsubsection{Dynamic Fill}

A typical dynamic capillary fill experiment was conducted by attaching rubber tubing from a large fluid reservoir $(\sim 2 \mathrm{~L})$ to the end of the capillary. The reservoir height was set equal to the bottom of the capillary, which was indicated by a blue dot upon the rubber tubing. The height of the capillary was maintained at a fixed position, while the height of the reservoir was varied in $2 \mathrm{~mm}$ increments. The reservoir height was initially set equal to the bottom of the capillary, which was indicated by a blue dot upon the rubber tubing. The system was allowed to equilibrate for at least two minutes between 
height adjustments. The difference between the reservoir height and capillary fluid fill was recorded.

\subsubsection{Purging}

A typical self-purging capillary fill experiment was conducted by placing a hydrophobic capillary within a reservoir slightly below the fluid surface to eliminate exit wetting effects and hydrostatic backpressure as displayed in Figure 2.13. A syringe was used to draw fluid into the capillary, partially filling the capillary. The syringe was then removed, exposing the free surface of the fluid column to atmospheric pressure.
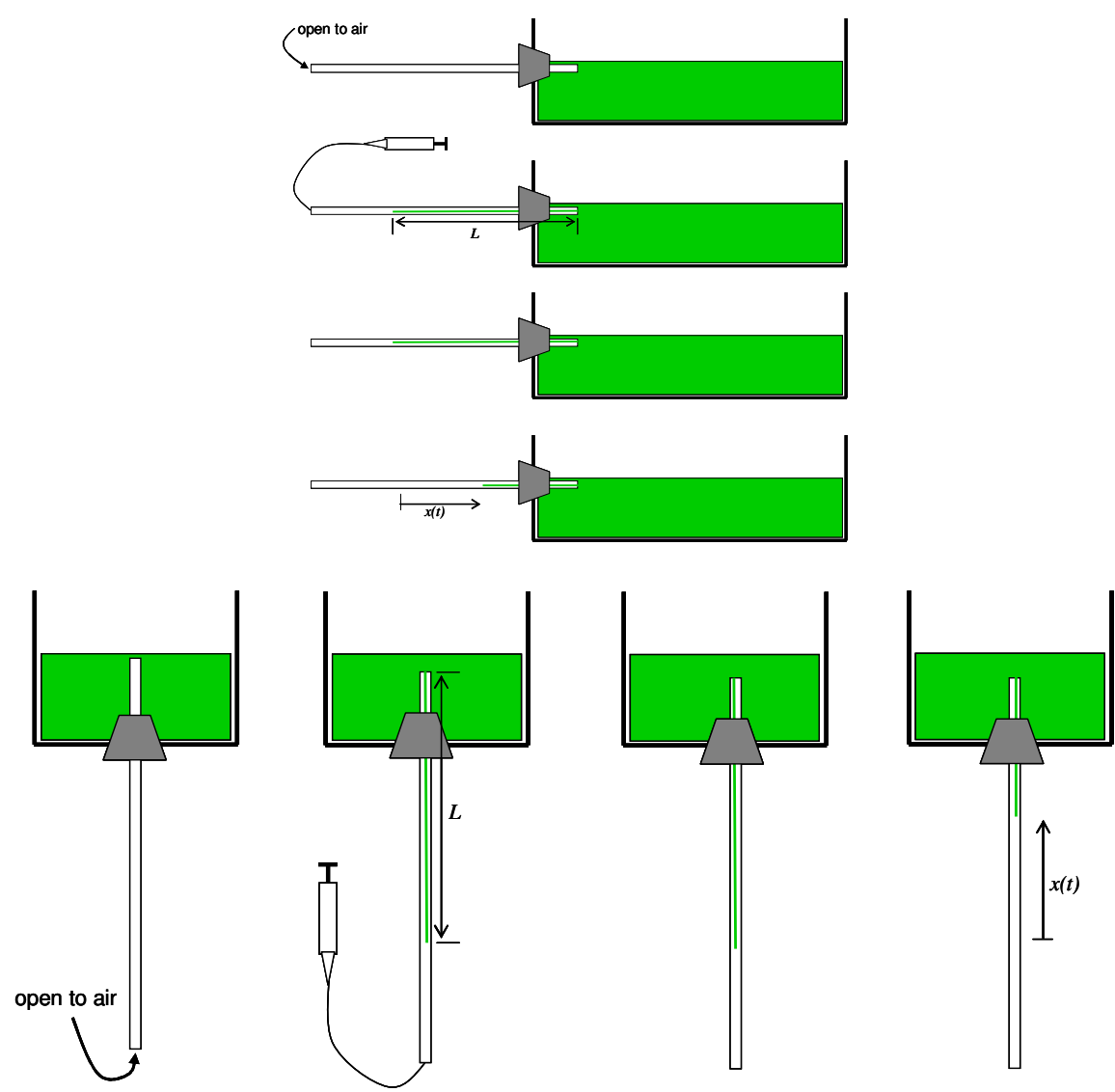

Figure 2.13 Schematic representations of the self-purging capillary experiments. 
(1) Trask, R. S.; Bond, I. P. Smart Mat. Struct. 2002, 15, 704-710.

(2) Trask, R. S.; Bond, I. P.; Williams, G. J. J. Roy. Soc. Interface 2006, 4, 363-371.

(3) Bond, I. P.; Pang, J. W. C. Composites 2005, 36, 183-188.

(4) Therriault, D.; White, S. R.; Lewis, J. A. Nat. Mater. 2003, 2, 265-271.

(5) Therriault, D.; White, S. R.; Lewis, J. A.; Shepher, R. F. Adv. Mater. 2005, 17, 395-399.

(6) Franke, T. A.; Wixforth, A. ChemPhysChem 2008, 9, 2140-2156.

(7) Whitesides, G. M. Nature 2006, 442, 368-373.

(8) Wheeler, T. D.; Stroock, A. D. Nature 2008, 455, 208-212.

(9) Liang, L.; Feng, X.; Liu, J.; Rieke, P. C.; Fryxell, G. E. Macromolecules 1998, 31, 7845-7850.

(10) Suk, J.; Cho, J. J. Micromech. Microeng. 2007, 17, N11-N15.

(11) Caprioli, L.; Mele, E.; Angile, F. E.; Girardo, S.; Athanassiou, A.; Camposeo, A.; Cingolani, R.; Pisignano, D. App. Phys. Lett. 2007, 91, 110-113.

(12) Carruthers, W. Cycloaddition Reactions in Organic Synthesis Oxford, U. K., 1990.

(13) Fringuelli, F.; Taticchi, A. Dienes in the Diels-Alder Reaction; John Wiley \& Sons: New York, 1990.

(14) Costanzo, P. J.; Demaree, J. D.; Beyer, F. L. Langmuir 2006, 22, 10251-10257.

(15) Costanzo, P. J.; Beyer, F. L. Macromolecules 2007, 40, 3996-4001.

(16) Costanzo, P. J.; Beyer, F. L. Chem. Mater. 2007, 19, 6168-6173.

(17) Imai, Y.; Itoh, H.; Naka, K.; Chujo, Y. Macromolecules 2000, 33, 4343-4346.

(18) Gheneim, R.; Perez-Berumen, C.; Gandini, A. Macromolecules 2002, 35, 72467253.

(19) Chen, X.; Wudl, F.; Mak, A. K.; Shen, H.; Nutt, S. R. Macromolecules 2003, 36, 1802-1807.

(20) Mcelhanon, J. R.; Russick, E. M.; Wheeler, D. R.; Low, D. A.; Aubert, J. H. J. Appl. Poly. Sci. 2002, 85, 1496-1502.

(21) Naud, C.; Calas, P.; Commeyras, A. Langmuir 2001, 17, 4851-4857.

(22) Kashid, M. N.; Agar, D. W. Chem. Eng. J. 2007, 131, 1-13.

(23) Jensen, R. E.; Palmese, G. R.; McKnight, S. H. International Journal of Adhesion and Adhesives 2006, 26, 103-115.

(24) Lee, I.; Wool, R. P. Macromolecules 2000, 33, 2680-2687.

(25) Park, J. O.; Jang, S. H. J. Poly. Sci. A 1992, 30, 723-729. 


\section{Aligned Poly(3,4-alkyleneoxythiophene) Brushes on Indium-Tin Oxide from Surface Initiated Atom Transfer Radical Polymerization and Subsequent Electro- Polymerization.}

\subsection{Introduction}

\subsubsection{Motivation}

The world is experiencing an exponential increase in energy consumption. This increase is driven by population growth and by the ever increasing energy demand of the 3 billion lesser energy users in the non-legacy world. ${ }^{1}$ Current predictions illustrate that by 2050 the energy need will be roughly double that of current global usage and by the beginning of the $22^{\text {nd }}$ century the requirement will be triple. ${ }^{2}$ This daunting energy crisis can be largely regarded as the number one issue that humanity, and most notably the younger generation of the current populus will have to face in the coming years. ${ }^{3}$

Such a scenario presents two major tribulations. The first is that with roughly $80 \%$ of current energy production coming from fossil fuels and their inevitable depletion, a need for an efficient renewable energy source is desperately necessary. The second problem is that if fossil fuels are consumed at current or even greater levels the $\mathrm{CO}_{2}$ introduction to the environment will reach catastrophic levels. ${ }^{4,5}$ To properly address the projected need with current renewable energy production sources such as wind power, hydroelectric, nuclear or biomass is simply an impossible feat. For example, the entire land mass capable of wind-on-land energy production would have to be saturated with windmills, the whole of the world's agricultural land mass would have to be used in biomass production, a new nuclear power plant would have to be built every other day, 
and every available river would have to be dammed for hydroelectric power in order to meet the projected needs with minimal carbon impact. ${ }^{4}$

The long term answer to this dilemma can be found in solar energy ${ }^{1,4-6}$ as shown in Table 3.1 which displays the energy theoretically available for harvest from major renewable sources. ${ }^{7}$ Thus there is the need to develop affordable, efficient, easy to manufacture photovoltaic technology that is capable of harvesting the abundant solar energy which bombards the surface of the earth on a daily basis.

Table 3.1 Theoretical Energy Potential of Various Renewable Energy Resources. ${ }^{7}$

\begin{tabular}{cc}
\hline Resource & Theoretical Energy Potential (GW) \\
\hline Photovoltaics (Solar Power) & 206000 \\
Wind & 11000 \\
Geothermal & 663 \\
Hydroelectric & 140 \\
Biomass & 78 \\
\hline
\end{tabular}

\subsubsection{Current Technologies}

\subsubsection{Inorganic Solar Cells}

There are a number of materials capable of functioning as a solar absorber for use in photovoltaics as well as many different device architectures capable of housing such materials in order to successfully convert solar radiation into electricity. ${ }^{8}$ Currently single- and multicrystalline silicon solar cells are the most widely implemented varietal of photovoltaic cell. ${ }^{9} \quad$ The current preference for inorganic based solar cells stems from their abilies to absorb radiation over a broad section of the electromagnetic spectrum, and facilitate high charge carrier mobility, as well as the overall stability of the materials which they are contructed from. ${ }^{8}$ 
The band gap of silicon is $1.11 \mathrm{eV}$, which sits enticingly close to the optimized value for photoconversion of $1.3 \mathrm{eV}{ }^{10}$ Thus theoretical maximum solar efficiency of cells based on $\mathrm{Si}$ is respectively high at $42 \% .^{11}$ Yet silicon has a very low absorption coefficient that requires the thickness of Si to be on the order of $100 \mu \mathrm{m}$ in order to sufficiently absorb the solar spectrum. Consequently the methods required to produce high purity Si deposited on a surface at such thicknesses requires high temperature purification and vapor deposition techniques that are considerable in energy and material costs. ${ }^{12}$ Organic based solar cells offer considerable advantages in the area of material production costs and processability. However; organic photovoltaics have only achieved an $\sim 8 \%$ efficiency compared to that of Si based cells at $\sim 28 \%$.

\subsubsection{Organic Photovoltaics (OPVs)}

A compound that possesses an electronic structure consisting of a delocalized $\pi$ electron system is capable of absorbing sunlight and subsequently the creation and transport of photogenerated charge carriers. ${ }^{13}$ When the charge carriers, which appear in the form of hole-electron pairs (excitons), are created there is a need for dissociation of the two charged constituents prior to recombination. If this is not accomplished the photovoltaic cell will not function. Unfortunately the binding energy of the exciton is very high in organic semiconductors compared to that of inorganic based materials that are observed to facilitate immediate dissociation. ${ }^{14}$

The model architecture of an OPV cell is displayed by Figure 3.1. Generally an OPV cell consists of 5 main constituents. The first of these is a substrate supported transparent anode. Second is a transparent, conductive polymer layer that functions as a 



Figure 3.1 Basic OPV cell structure.

contact for the anode. The optimization of this conductive polymer layer is the focus of the current study. The middle section of the device is the photoactive layer where the main photophysical processes occur. Finally is the hole blocking layer and the cathode.

\subsection{Indium Tin-Oxide Transparent Anode}

The transparent anode that is widely implemented is indium tin-oxide (ITO) supported on a glass substrate. ${ }^{15}$ ITO thin films are crucial components in numerous optoelectronic devices other than photovoltaics such as light-emitting diodes which utilize photoactive organic materials. ${ }^{16-18}$ The optimized performance of devices that implement ITO thin films as a transparent anode is critically dependent upon incorporation of an organic based material at the ITO electrode interface. ${ }^{19,20}$ These interfaces of indium tin-oxide layer with organic based photoactive thin films has been 
extensively studied allowing for a good understanding of the complicated surface science inherent of the material. ${ }^{21}$

There are numerous difficulties associated with the modification of ITO via interfacial coupling chemistry aimed at maximizing structure-property correlations for inclusion in devices. This is largely due to a great degree of surface heterogeneity causing only a small proportion of the surface being electroactive. The modification of the surface of ITO with both insulating and conductive polymers has been investigated as the organic material interface. These materials were often incorporated via electropolymerization initiated at the electrode surface. ${ }^{22,23}$ Further it has been reported that when electropolymerization is undertaken on ITO surfaces modified with a polymerizable monolayer that the resulting polymeric thin films exhibit enhanced "wiring" across the organic/inorganic interface. ${ }^{24}$ While the electropolymerization route to modifying ITO electrodes has proved to be very useful it has also been demonstrated that growing tethered polymers from ITO utilizing controlled radical polymerization techniques such as surface initiated atom transfer radical polymerization (SI-ATRP) allows for better tailoring of polymer molecular weight, functionality and composition. $^{16,25,26}$

\subsection{Photoactive Layer}

Different photoactive layer architectures bilayer device (Figure 3.2a) utilizes a photoactive layer analagous to a conventional p-type/n-type electron donor/acceptor setup. ${ }^{13}$ The photoactive layer is sandwiched in between a conductive polymer layer and two electrodes one of which is transparent. The charged species of are only capable of 
traveling 10-20 nm prior to recombination and thus in a bilayer device only excitons generated very close to the interface of the photoactive layer are capable of separation and transport to their respective electrodes.

To alleviate the hindrance on efficiency of having such a limited interfacial area capable of effectively separating the charged species of the photogenerated exciton a system with a drastically larger surface area between the electron donor and acceptor material is desired. Figure $3.2 \mathrm{~b}$ and $3.2 \mathrm{c}$ depict devices constructed with disordered and

a)

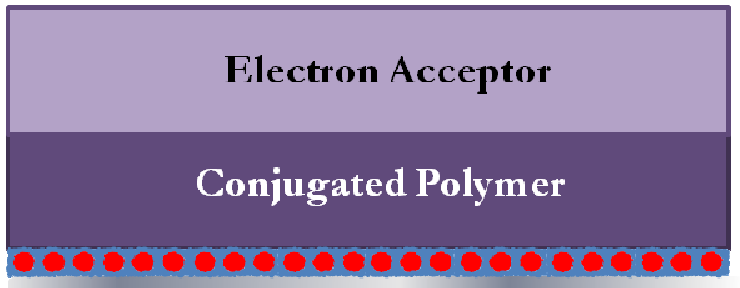

ITO

Bilayer

b)



ITO

Disordered Bnlk I leterojunction

c)

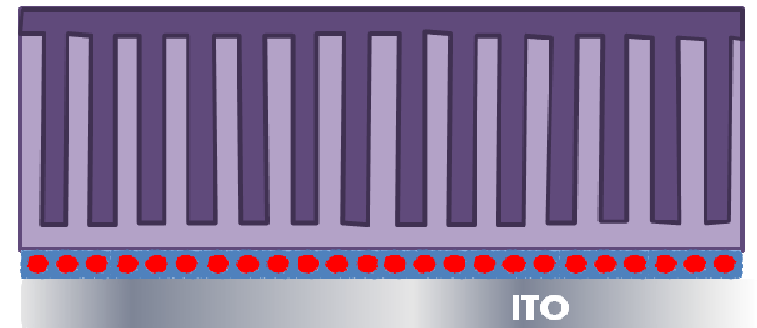

Ordered Bullk Heterojunction

Figure 3.2 Schematics of bilayer (a), disordered bulk heterojunction (b) and ordered bulk heterojunction (c) photoactive layers. 
ordered bulk heterojunction photoactive layers respectively. The latter is the optimal design but such a complicated ordered morphology is extremely difficult to construct at the nano-scale dimensions desired and thus the disordered analog is utilized as it can be created via simple deposition of a blend of the donor and acceptor materials.


PVT
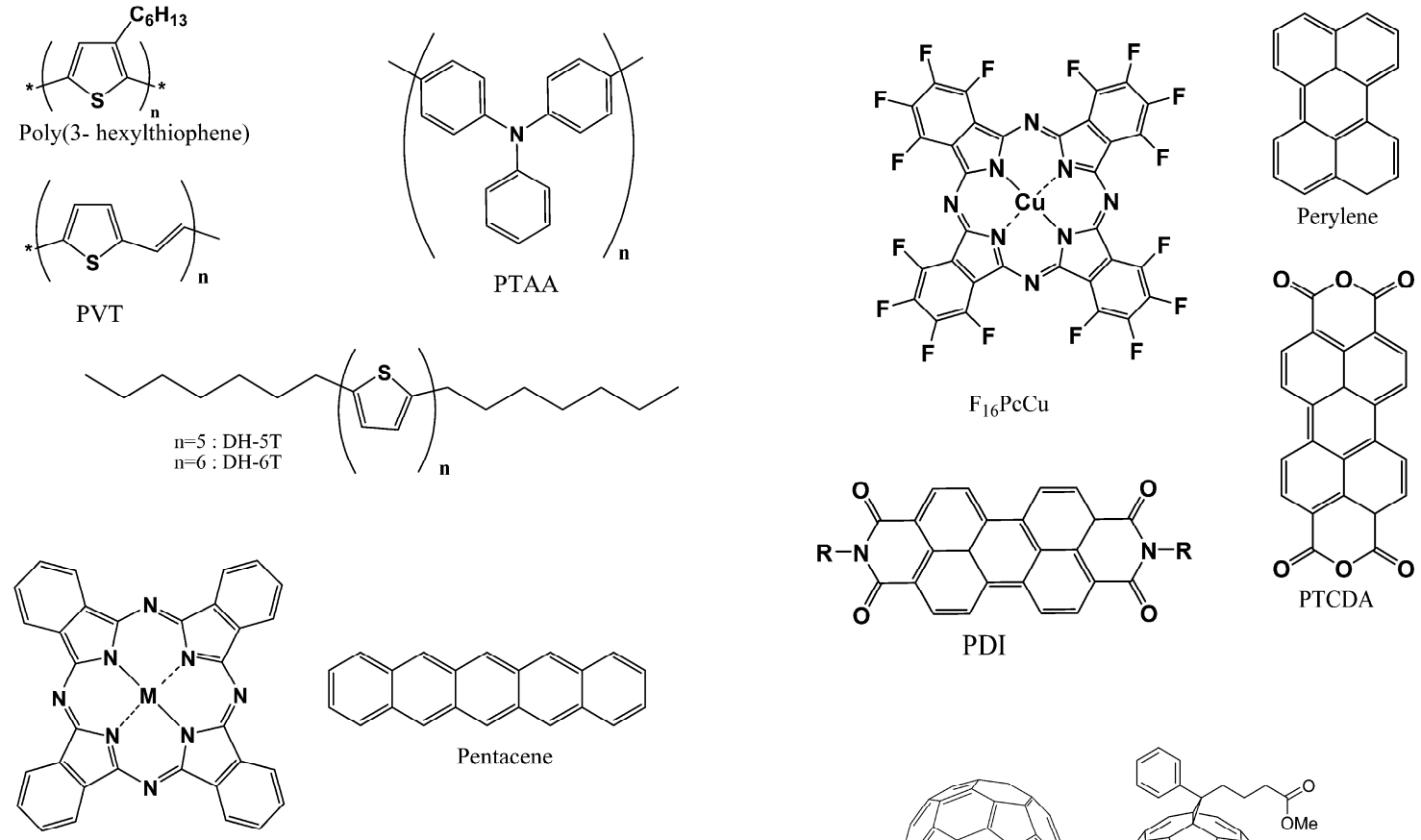

Phthalocyanine

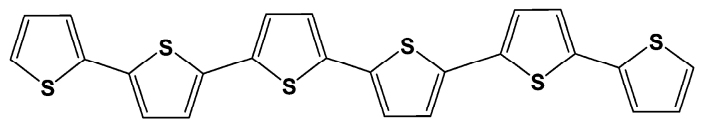

$\alpha$-sexithiophene

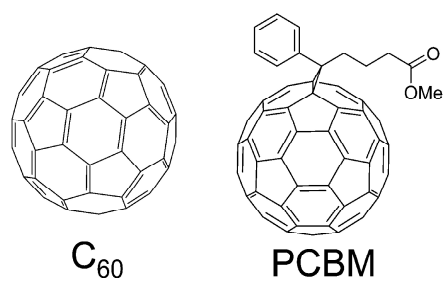

Figure 3.3 Examples of organic semi-conductors used in organic solar cells. 
A number of compounds that have been implemented as organic semi conductor materials for use in the photoactive layer are shown in Figure 3.3. The most common family of compounds used as the conjucated polymer, electron donor material are poly(thiophene variants) and fullerene derivatives are utilized as the electron acceptor material.

\subsection{Conductive Polymer Layer}

There is a need for a charge transport layer that effectively blocks electrons from reaching the ITO electrode while improving the contact between the anode and photoactive layer of the device. The material that is most commonly employed for this purpose is poly(3,4-ethylenedioxythiophene):poly(styrenesulfonate) (PEDOT:PSS) which is an oxidataively doped, cationic conducting polythiophene variant (PEDOT) that is electrostatically bound to a polyanion (PSS). ${ }^{27}$ The properties of this material, as with all conduting and semi-conducting $\pi$-con jugated polymer materials, are heavily influenced by the morphology at the micro and nano scale. ${ }^{28}$

As depicted by Figure 3.4 the morphology of spin cast or solution deposited PEDOT:PSS films is a phase segregated material consisting of PEDOT nanodomains impregnated in an excess of PSS. ${ }^{29}$ The PEDOT regions posses a notable higher intrinsic conductivity as PSS is only a poor ionic conductor. This presents the issue that electron transport must occur between the isolated PEDOT grains ${ }^{27}$ which is not an optimal situation for maximized charge transport efficiency. 


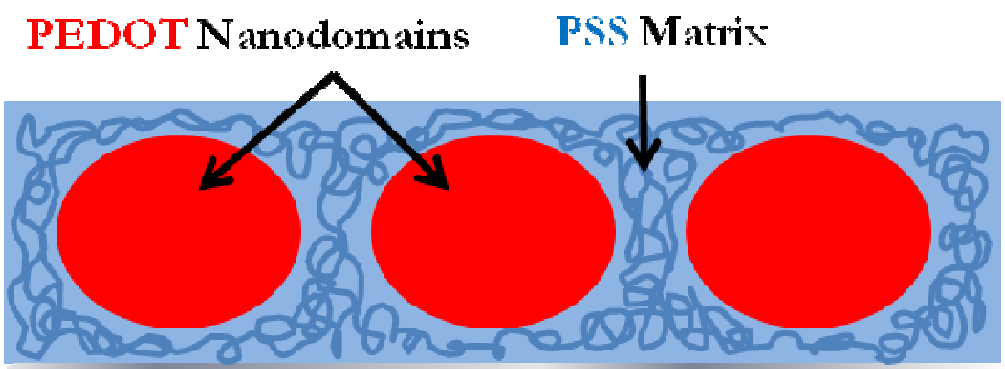

ITO
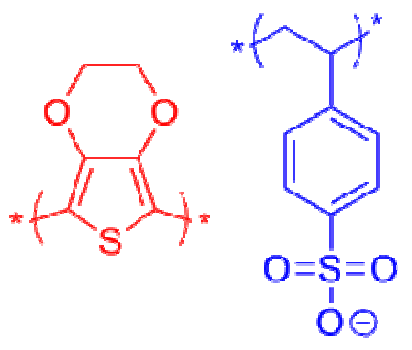

PEDOT

\section{PSS}

Figure 3.4 Morphology of PEDOT:PSS transparent conductive polymer layer cast on an indium tin-oxide substrate.

\subsubsection{Experimental Strategy}

In the present study a PEDOT:PSS layer with a morphology that optimizes the charge transport ability of the material was developed. A conductive layer created by grafting a poly(styrene) brush via a controlled radical polymerization method such as surface initiated atom transfer radical polymerization (SI-ATRP) that carries electropolymerizable thiophene groups where the extended, sterically stretched chains can template the formation of aligned polythiophene chains provides a route to the creation of a conductive polymer layer that is not phase segregated into nano domains of conductive, and non-conductive sites.

By first utilizing a 3,4-propylenedioxythiophene (ProDOT) functional styrenic monomer to complete SI-ATRP from an ATRP initiator functionalized ITO surface followed by electropolymerization of the ProDOT functional side chains a stepwise process for the surface modification ITO is achieved. This stepwise functionaliztion allows for the creation of well defined, aligned, poly(3,4-alkyleneoxythiophene) brushes from ITO. The morphology of a conductive polymer layer created by this synthetic methodology is depicted by Figure 3.5. 


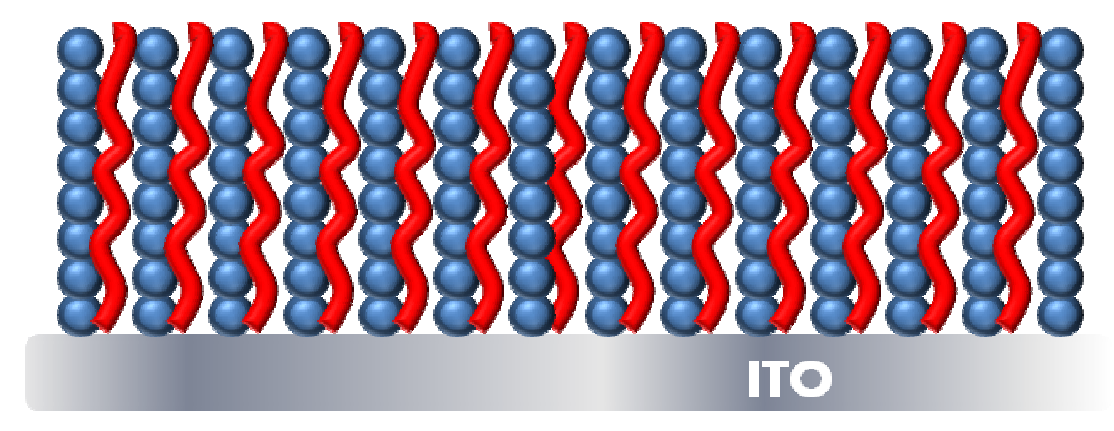

Figure 3.5 Design of conductive poly(thiophene) layer from SI-ATRP and subsequent electropolymerization. Blue constituents represent the poly(styrene) backbone and red species represent the aligned, conductive poly(thiophene) chains.

\subsection{Results and Discussion}

\subsubsection{Stepwise Surface Modification of ITO}

Scheme 2 illustrates the synthetic methodology implemented for the modification of an ITO surface with aligned poly(3,4-alkyleneoxythiophene) brushes. The initial step in the functionalization of the ITO surface involved the synthesis (Scheme 1) and immobilization of a phosphonic acid ATRP initiator (1). SI-ATRP was then completed with a ProDOT/Styrene hybrid monomer (ProDOT-Sty, 2) prepared as described in Scheme 3. The SI-ATRP was run in tandem with a solution ATRP by including free

Scheme 3.1. Preparation of 4-(bromomethyl)benzylphosphonic acid<smiles>CCOP(=O)(Cc1ccc(CBr)cc1)OCC</smiles> 
Scheme 3.2. Synthetic Route for Preparation of Aligned Poly(3,4-alkyleneoxythiophene) Brushes from Indium Tin Oxide Surfaces. ${ }^{A}$

\section{Unfunctionalized Electrode \\ ATRP Initiator \\ Functionalized \\ Electrode and \\ Sacrificial Imitiator}

ProDOT Functional

Polymer Brush and Free

I inear Polymer
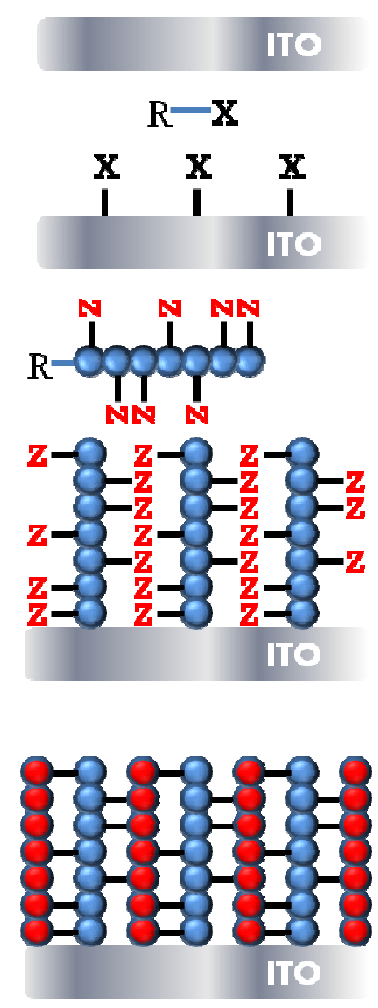

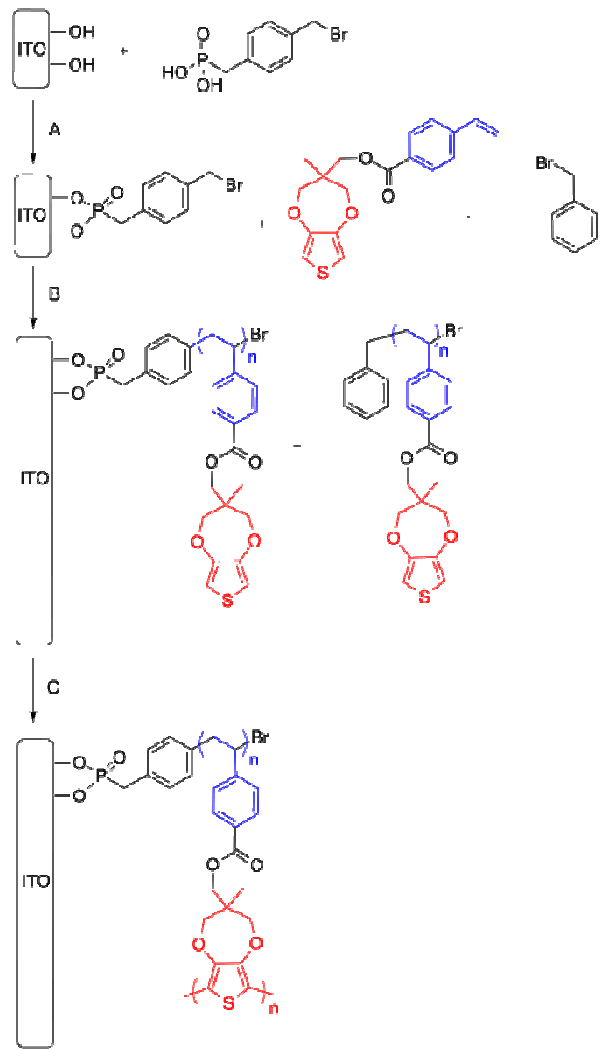

${ }^{A}$ Conditions: Conditions: A) Heat B) $\mathrm{Cu}^{\mathrm{I}} \mathrm{Br}$, PMDETA, Anisole $100{ }^{\circ} \mathrm{C}$ C) Electropolymerization

Scheme 3.3. Synthetic Route for Preparation of ProDOT-Sty<smiles>C=Cc1ccc(C(=O)OCC2(C)COc3cscc3OC(C)(CO)C2)cc1</smiles>

"sacrificial" initiator in the system in order to provide free polymer that is capable of easy isolation for characterization purposes. Subsequent to the SI-ATRP of ProDOT-Sty the 
pendant ProDOT moiety on each repeat unit of the aligned polymer brushes was incorporated into a conductive poly(3,4-alkyleneoxythiophene) network via electropolymerization initiated via the application of a potential across the ITO electrode.

\subsubsection{Patterning of ATRP Initiator Functionalized ITO}

It has been established that there is a linear relationship of polymer brush thickness with free polymer molecular weight for a controlled SI-ATRP from ITO. ${ }^{16}$ Therefore ATRP initiator functionalized ITO samples capable of growing brushes in a patterned manner were prepared in order to facilitate for brush thickness characterization

Scheme 3.4. Photopatterning of ATRP initiator functionalized ITO
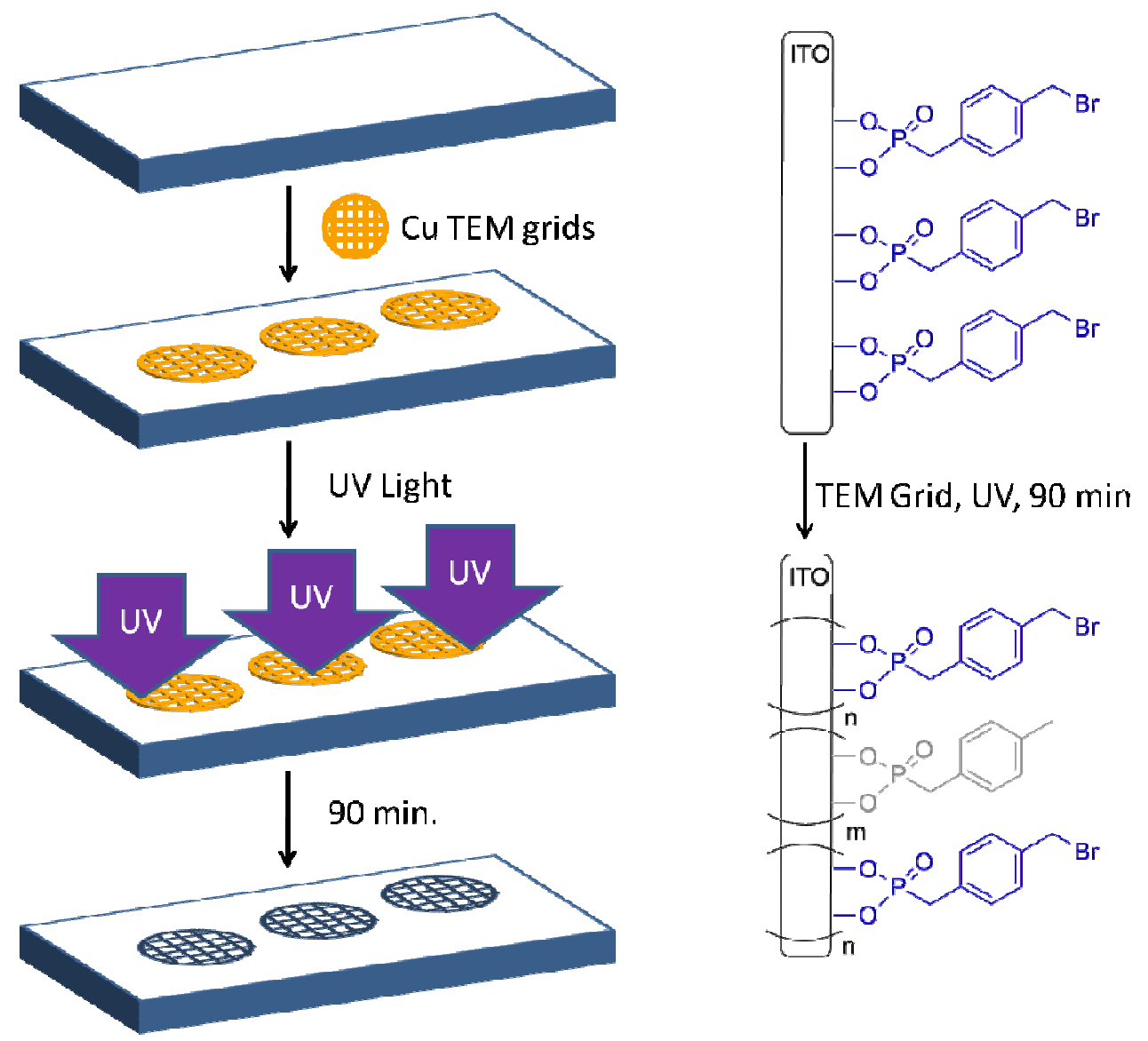
via atomic force microscopy (AFM). This was accomplished by first functionalizing the ITO substrate as described in Scheme 2A and then performing lithography as shown by Scheme 4 where $\mathrm{Cu}$ TEM grids were implemented as masks prior to irradiation of the surface with ultraviolet light (UV). The successful elimination of the bromo functionality from the ATRP initiator functionalized surface has been confirmed via X-ray photoelectron spectroscopy. ${ }^{16}$ Figure 3.6 displays the expanded XPS spectrum of $\mathrm{Br} 3 \mathrm{~d}$ of ATRP initiator functionalized ITO over 90 min of UV irradiation. ${ }^{16}$ Scanning electron microscopy (SEM) was also utilized to confirm that the photopatterning process was successful. Figure 3.7 displays SEM micrographs of the photopatterned ITO surface.

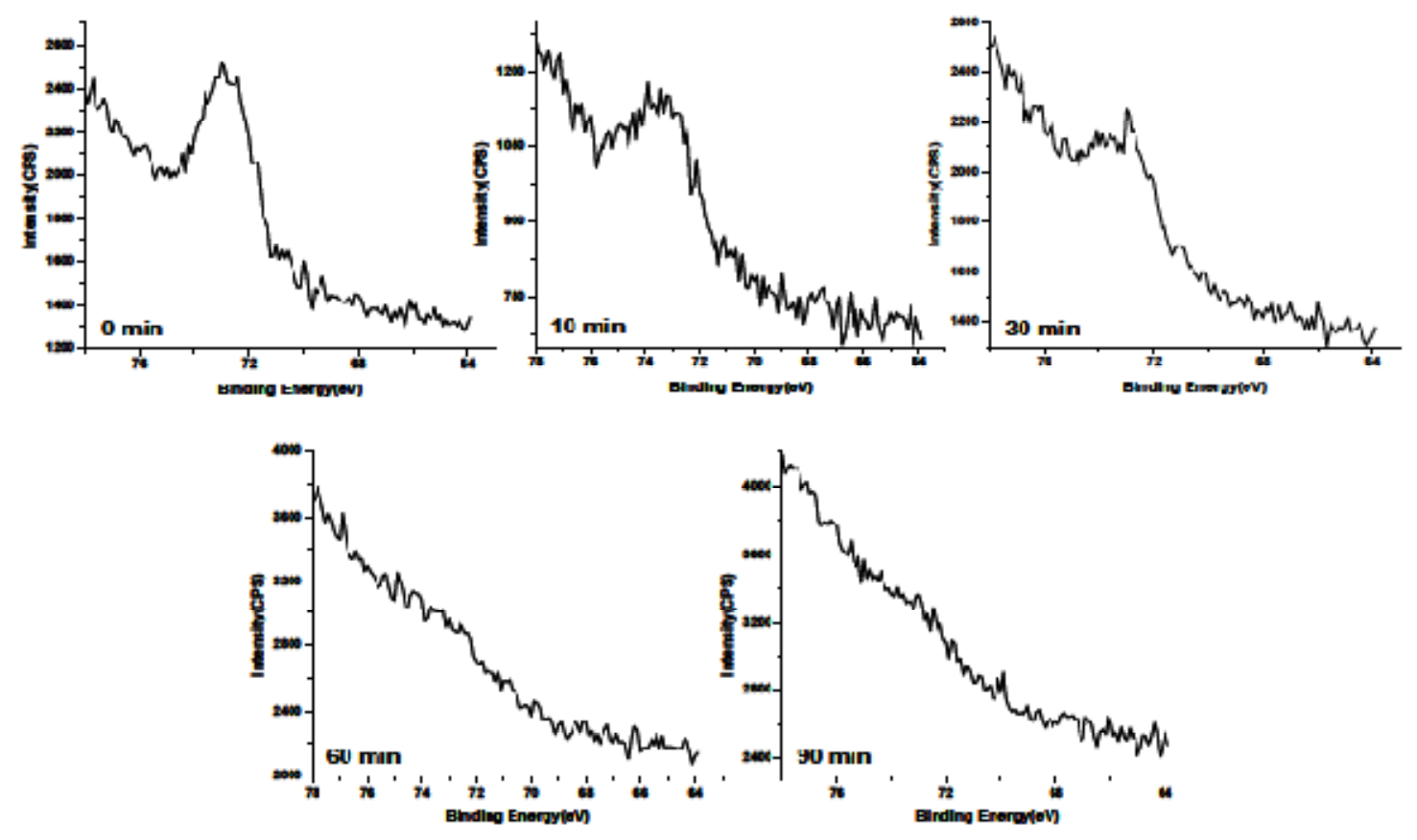

Figure 3.6 Expanded XPS spectrum of $\mathrm{Br}$ 3d of ATRP initiator functionalized ITO over 90 minutes of UV irradiation. Reproduced from Kim et. al. ${ }^{16}$ 


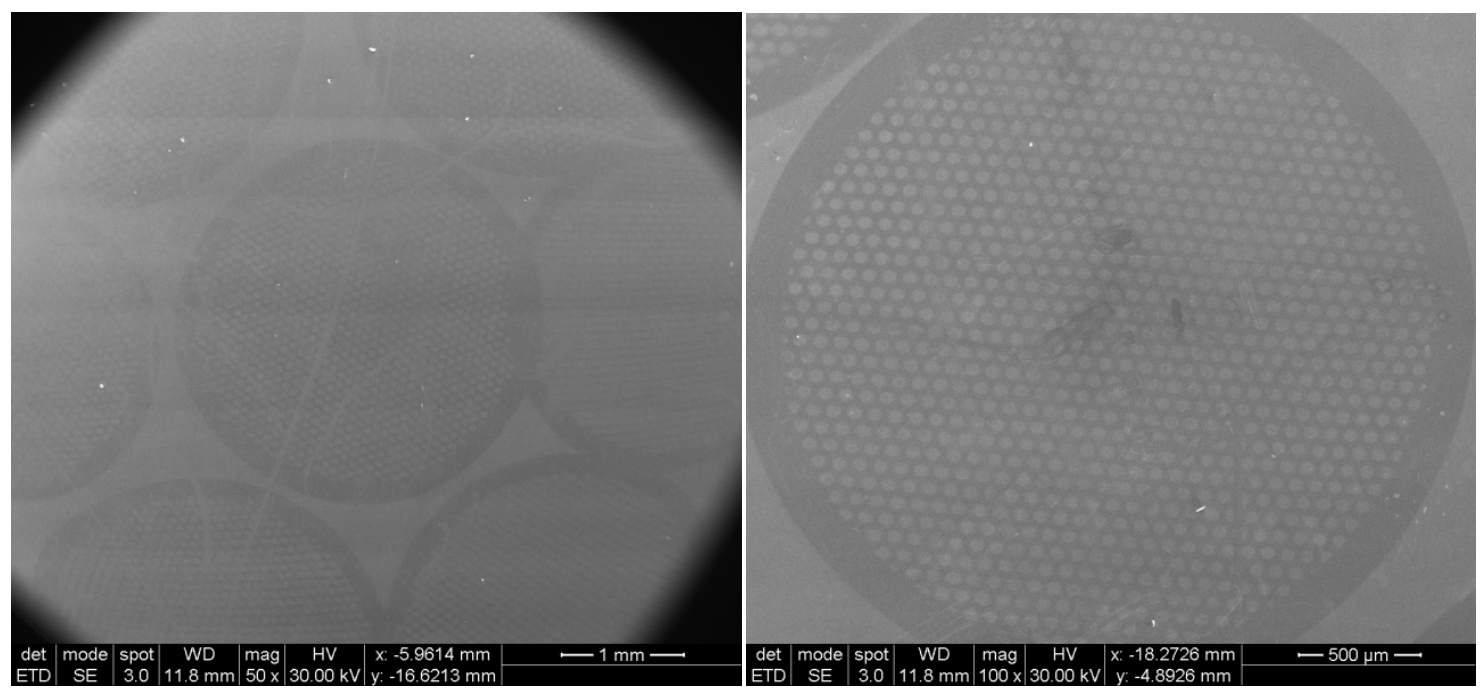

Figure 3.7 SEM micrographs of photopatterned ITO

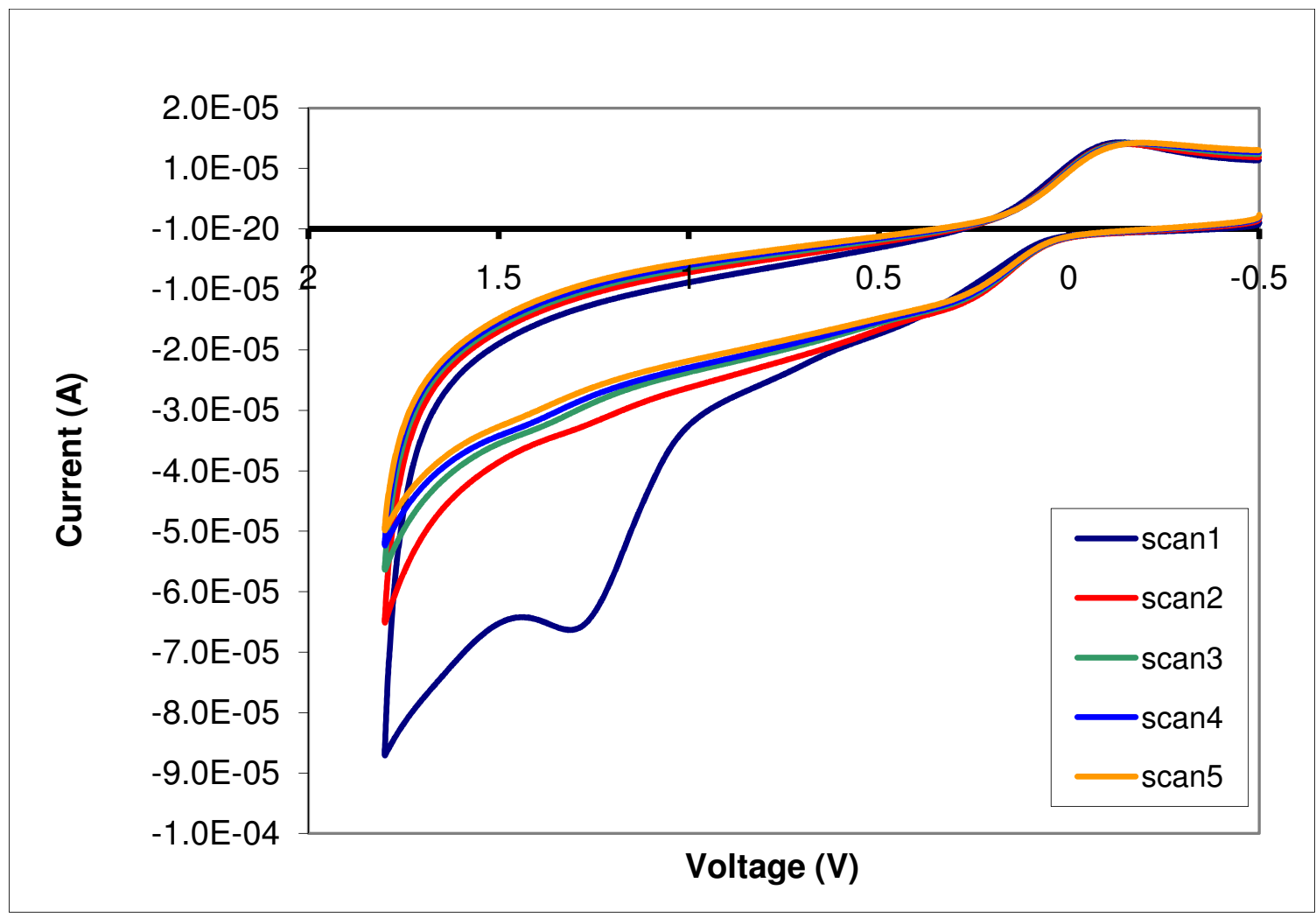

Figure 3.8 Cyclic voltammogram of poly(ProDOT-Sty) grafted from ITO referenced against $\mathrm{Fc} / \mathrm{Fc}^{+}$ 


\subsubsection{Electropolymerization of ProDOT Side Chain}

Cyclic voltammetry (CV) was utilized to monitor the electropolymerization of the pendant ProDOT groups attached to the poly(styrene) backbone. Figure 3.8 displays the cyclic voltammogram of an ITO electrode grafted with poly(ProDOT-Sty) brushes. The electrochemical event observed in the first scan beginning at approximately $+0.7 \mathrm{~V}$ vs ferrocene $(\mathrm{Fc}) / \mathrm{Fc}^{+}$is attributed to the electropolymerization of the ProDOT groups. This is in agreement with the literature on the electropolymerization of 3,4alkyleneoxythiophene species. ${ }^{30}$ Subsequent scans elucidate that the polymerization is not a reversible event as indicated by no observable event occurring again around $+0.7 \mathrm{~V}$.

\subsection{Conclusions}

A novel ProDOT/styrene hybrid monomer was synthesized and successfully utilized for the creation of aligned poly(3,4-alkyleneoxythiophene) brushes on indium-tin oxide via surface initiated atom transfer radical polymerization and subsequent electropolymerization. Photopatterning of the ATRP intiator functionalized ITO substrate, for the facilitation of later polymer brush height characterization via AFM, was conducting via lithography with $\mathrm{Cu}$ TEM grids acting as a mask during UV irradiation of the surface. The patterning was confirmed via SEM and XPS characterization of the surface. Cyclic voltammetry of an ITO electrode grafted with poly(ProDOT-Sty) brushes confirmed the successful electropolymerization of pendant ProDOT groups along the poly(styrene) brush backbone.

Future work will focus on the creation of a series of poly(ProDOT-Sty) brushes with varied heights. These sample will be used to establish a correlation between free 
polymer chain molecular weight and polymer brush height in order to confirm the true controlled nature of the surface initiated polymerization. They will also serve to provide quantitative data on the electropolymerization event via $\mathrm{CV}$ characterization. Finally separate devices will be created utilizing both the novel transparent conductive polymer layer based on ProDOT-Sty and the current standard of deposited PEDOT:PSS. The efficiencies of the different devices will be compared to quantify the performance gain resultant to implementation of the aligned poly(3,4-alkyleneoxythiophene) transparent conductive polymer system.

\subsection{Experimental}

\subsubsection{Materials}

All materials were acquired from commerically available sources and used without further purification.

\subsubsection{Instrumentation}

${ }^{1} \mathrm{H}$ and ${ }^{13} \mathrm{C}$ NMR was conducted on either a $400 \mathrm{MHz}$ Bruker or $600 \mathrm{MHz}$ Varian spectrometer in $\mathrm{CDCl}_{3}$, chemical shifts are reported as $\delta(\mathrm{ppm})$, referenced to the residual solvent peak. Gel permeation chromatography (GPC) was performed in a tetrahydrofuran mobile phase with a Waters 1515 isocratic pump running three $5 \mu \mathrm{m}$


differential refractometer and Waters 2487 dual wavelength UV-Vis spectrometer. Molar masses were calculated using the Empower software (Waters) calibrating against low polydispersity linear polystyrene standards. High resolution mass spectrometry (HRMS) 
was conducted by the Mass Spectrometry Facility of the University of Arizona Department of Chemistry and Biochemistry. Electrochemical measurements were made with a $\mathrm{CH}$-Instruments 660c potentiostat/galvanostat in a homebuilt Teflon compression cell. SEM images were taken on a Hitachi 4800 FE-SEM on as-prepared samples. XPS characterization was performed on a KRATOS 165 Ultra photoelectron spectrometer, using a monochromatic $\mathrm{Al} \mathrm{K} \alpha$ radiation source (20eV pass energy) at $1486.6 \mathrm{eV}$.

\subsubsection{Synthetic Preparations}

3.4.3.1 Synthesis of (3-methyl-3,4-dihydro-2H-thieno[3,4-b][1,4]dioxepin-3-yl)methanol [ProDOT-OH]

A 3-neck $1000 \mathrm{~mL}$ round bottom flask equipped with a magnetic stirbar and condenser was loaded with toluene $(750 \mathrm{~mL})$. While stirring 3,4-dimethoxythiophene (3.3 mL, $27.8 \mathrm{mmol}), 1,1,1$-tris(hydroxymethyl) ethane $(4.32 \mathrm{~g}, 36.1 \mathrm{mmol})$ and ptoluenesulfonic acid $(0.52 \mathrm{~g}, 2.78 \mathrm{mmol})$ were added. The reaction was stirred at $100^{\circ} \mathrm{C}$ with temperature control via thermocouple for 48 hours. The solvent was removed in vacuo and the viscous black residue taken back up in EtOAc and washed with deionized water $\mathrm{x} 3$. The solution was then reconcentrated in vacuo and the crude product was purified via $\mathrm{SiO}_{2}$ column chromatography (4:1 hexanes:EtOAc) to yield ProDOT-OH as

a brown oil that later solidified to a dark green/brown solid ( $3.4 \mathrm{~g}, 65 \%$ yield). ${ }^{1} \mathrm{H}$ NMR: $\delta 6.48(\mathrm{~s}, 2 \mathrm{H}), 4.08(\mathrm{~d}, J=11.9 \mathrm{~Hz}, 2 \mathrm{H}), 3.75(\mathrm{~s}, 2 \mathrm{H}), 3.74(\mathrm{~d}, J=11.9 \mathrm{~Hz}, 2 \mathrm{H}), 0.95(\mathrm{~s}$, $3 \mathrm{H})$. 
3.4.3.2. Synthesis of (3-methyl-3,4-dihydro-2H-thieno[3,4-b][1,4]dioxepin-3-yl)methyl 4-vinylbenzoate [ProDOT-Sty, 2]

A $250 \mathrm{~mL}$ round bottom flask equipped with a magnetic stirbar was loaded with 4-vinylbenzoic acid (1.917 g, $12.96 \mathrm{mmol})$. Anhydrous dichloromethane (30 mL) was added and the mixture was stirred until a homogenous solution resulted. The solution was then cooled to $0^{\circ} \mathrm{C}$ with an ice bath and dicyclohexylcarbodiimide $(2.674 \mathrm{~g}, 12.96$ mmol) and 4-(dimethylamino)pyridine (cat., $144 \mathrm{mg}, 1.17 \mathrm{mmol}$ ) were added. The solution was stirred at $0^{\circ} \mathrm{C}$ for $15 \mathrm{~min}$ before ProDOT-OH $(2.190 \mathrm{~g}, 10.18 \mathrm{mmol})$ in anhydrous $\mathrm{CH}_{2} \mathrm{Cl}_{2}(36 \mathrm{~mL})$ was added dropwise. The reaction was allowed to come to room temperature while stirring for $16 \mathrm{hrs}$. The urea precipitate was filtered off and resulting solution concentrated in vacuo. The residue was taken back up into EtOAc and cooled to facilitate the precipitation of any remaining urea which was again filtered off. The compound was purified via $\mathrm{SiO}_{2}$ column chromatography (10\% EtOAc in hexanes) to yield 2 as a colorless oil that later crystallized to a white solid (3.3 g, 81\% yield). ${ }^{1} \mathrm{H}$ NMR: $\delta 7.99(\mathrm{dt}, \mathrm{J}=8.4,2.0 \mathrm{~Hz}, 2 \mathrm{H}), 7.47(\mathrm{dt}, \mathrm{J}=8.4,2.0 \mathrm{~Hz}, 2 \mathrm{H}), 6.76(\mathrm{dd}, \mathrm{J}=17.6$, $10.9 \mathrm{~Hz}, 1 \mathrm{H}), 6.50(\mathrm{~s}, 1 \mathrm{H}), 5.87(\mathrm{dd}, \mathrm{J}=17.6,0.7 \mathrm{~Hz}, 1 \mathrm{H}), 5.40(\mathrm{dd}, \mathrm{J}=10.9,0.7 \mathrm{~Hz}$, 1H), $4.43(\mathrm{~s}, 1 \mathrm{H}), 4.14(\mathrm{~d}, \mathrm{~J}=12.0 \mathrm{~Hz}, 1 \mathrm{H}), 3.83(\mathrm{~d}, \mathrm{~J}=12.0 \mathrm{~Hz}, 1 \mathrm{H}), 1.08(\mathrm{~s}, 2 \mathrm{H}) .{ }^{13} \mathrm{C}$ NMR: $\delta 17.35,42.93,66.84,76.34,105.79,116.69,126.21,129.04,129.92,135.97$, 142.21, 149.61, 166.09. HRMS (ESI): $\mathrm{m} / z$ calculated for $\mathrm{C}_{18} \mathrm{H}_{19} \mathrm{O}_{4} \mathrm{~S}(\mathrm{M}+\mathrm{H})^{+}$331.0999, observed 331.1000 . 


\subsubsection{ATRP of ProDOT-Sty}

A $10 \mathrm{~mL}$ Schlenk flask equipped with a magnetic stirbar was loaded with ProDOT-Sty $(1.00 \mathrm{~g}, 3.03 \mathrm{mmol})$ and $\mathrm{Cu}(\mathrm{I}) \mathrm{Br}(21.5 \mathrm{mg}, 0.15 \mathrm{mmol})$. The flask was vacuum/backfilled with argon three times. Argon inlets were started on benzyl bromide $(17.8 \mu \mathrm{L}, 0.15 \mathrm{mmol})$ in anisole $(1 \mathrm{~mL})$ and $\mathrm{N}, \mathrm{N}, \mathrm{N}^{\prime}, \mathrm{N}^{\prime \prime}, \mathrm{N}^{\prime \prime}-$

pentamethyldiethylenetriamine $(15.6 \mu \mathrm{L}, 0.75 \mathrm{mmol})$ in anisole $(1 \mathrm{~mL})$ and allowed to purge with argon for $30 \mathrm{~min}$. The benzyl bromide and $\mathrm{N}, \mathrm{N}, \mathrm{N}^{\prime}, \mathrm{N}^{\prime \prime}, \mathrm{N}^{\prime \prime}-$

pentamethyldiethylenetriamine solutions were then added to the Schlenk flask via purged syringe. The mixture was degassed via three freeze/pump/thaw cycles and then placed in an oil bath thermostated to $100{ }^{\circ} \mathrm{C}$ and stirred for 2.5 hours. The resulting dark green solution was diluted with $\mathrm{CH}_{2} \mathrm{Cl}_{2}$ and passed through a short plug of basic alumina to remove any residual catalyst. The product was then recovered via precipitation into a large excess of rapidly stirred methanol. GPC: $M_{n}=8600 \mathrm{~g} / \mathrm{mol}, M_{w}=9200 \mathrm{~g} / \mathrm{mol}$, $\mathrm{PDI}=1.07$.

\subsubsection{Surface Modificiation}

3.4.4.1 ITO Modification with 4-(bromomethyl)benzylphosphonic acid

Indium Tin Oxide coated glass slides were scrubbed with an aqueous Triton X100 solution utilizing a microfiber cloth. They were then washed with DI water, nanopure water, and absolute EtOH and finally dried under an Ar stream. The slides were then placed in a slide treatment jar and sonicated in nanopure water (x2) for $10 \mathrm{~min}$ and absolute $\mathrm{EtOH}$ for $5 \mathrm{~min}$. The slides were then dipped into a $\mathrm{FeCl}_{3}$ solution $(2 \mathrm{M}$ in concentrated $\mathrm{HCl}$ ) for 10 seconds and subsequently rinsed with DI water and nanopure 
water. The slides were dried under Ar and allowed to rest for 3 min before submerging them into a solution of 4-(bromomethyl)benzyl phosphonic acid (4 $\mathrm{mM}$ in THF). The treatment was carried out for a minimum of 24 hours after which they were rinsed with THF and placed into an oven at $100{ }^{\circ} \mathrm{C}$ overnight. The slides were then sonicated in THF (x2) for $15 \mathrm{~min}$, and $\mathrm{EtOH}$ for $10 \mathrm{~min}$. The slides were patterned by placing $\mathrm{Cu}$ TEM grids (300 mesh, hexagonal) onto the substrate and irradiating with shortwave UV light for $90 \mathrm{~min}$. After irradiation the slides were sonicated in toluene for $30 \mathrm{~min}, \mathrm{CH}_{2} \mathrm{Cl}_{2}$ for $30 \mathrm{~min}$, and absolute EtOH for $15 \mathrm{~min}$. The slides were stored in absolute EtOH until used for SI-ATRP.

\subsubsection{SI-ATRP of 2}

A schlenk flask with 24/40 joint equipped with a stir bar was loaded with $\mathrm{Cu}^{\mathrm{I}} \mathrm{Br}$. The flask was vacuum/backfilled with $\operatorname{Ar}$ (x5). ProDOT-Sty was dissolved in anisole (9.5 mL). Ar inlets were started on the ProDOT-Sty solution, benzyl bromide, and $\mathrm{N}, \mathrm{N}, \mathrm{N}^{\prime}, \mathrm{N}^{\prime \prime}, \mathrm{N}^{\prime \prime}$-pentamethyldiethylenetriamine (PMDETA) and allowed to vigorously purge with Ar for 1 hour. The ProDOT-Sty solution and PMDETA were transferred to the reaction flask via purged syringe. The reaction mixture was stirred until a homogenous, light green solution was achieved. Benzyl bromide was then added via purged syringe and the system was degassed via freeze-pump-thaw cycles (x4). After the last freeze cycle, two modified ITO slides were introduced to the vessel under high Ar flow taking care to have the non ITO functionalized sides facing. The system was then deoxygenated by vacuum/backfilling with $\operatorname{Ar}(\mathrm{x} 3)$ over the frozen reaction mixture. The 
reaction was allowed to come to room temperature under Ar and placed in an oil bath thermostated to $100^{\circ} \mathrm{C}$. Slides were characterized via SEM and CV.

\subsection{References}

(1) Nocera, D. G. Inorganic Chemistry 2009, 48, 10001-10017.

(2) Hoffert, M. I.; Calderia, K.; Jain, A. K.; Haites, E. F.; Harvey, L. D. D.; Potter, S. D.; Schlesinger, M. E.; Scheider, S. H.; Watts, R. G.; Wigley, T. M. L.; Wuebbles, D. J. Nature 1998, 395, 881.

(3) Smalley, R. E. In Energy and Nanotechnology Rice University, 2003.

(4) Eisenberg, R.; Nocera, D. G. Inorganic Chemistry 2005, 44, 6799-6801.

(5) Hetterscheid, D. G. H.; Vlugt, J. I. v. d.; Bruin, B. d.; Reek, J. N. H. Angewandte Chemie International Edition 2009, 48, 8178.

(6) Nocera, D. G. Chemical Society Review 2009, 38, 13.

(7) NREL Renewable Energy Technology Resource Maps for the United States, 2009.

(8) Habas, S. E.; Platt, H. A. S.; Hest, M. F. A. M. v.; Ginley, D. S. Chemical Reviews 2010, 110, 6571-6594.

(9) Ginley, D.; Green, M. A.; Collins, R. MRS Bulletin 2008, 33, 355.

(10) Wadia, C.; Alivisatos, A. P.; Kammen, D. M. Environmental Science and Technology 2009, 43, 2072-2077.

(11) Shockley, W.; Queisser, H. J. Journal of Applied Physics 1961, 32, 510.

(12) Hibberd, C. J.; Chassaing, E.; Liu, W.; Mitzi, D. B.; Lincot, D.; Tiwari, A. N. Progress in Photovoltaics 2010, 18, 434-452.

(13) Gunes, S.; Neugebauer, H.; Saricifici, N. S. Chemical Reviews 2007, 107, 13241338.

(14) Po, R.; Maggini, M.; Camaioni, N. Journal of Physical Chemistry C 2010, 114, 695-706.

(15) Cahen, D.; Kahn, A. Advanced Materials 2003, 15, 271.

(16) Kim, B. Y.; Ratcliff, E. L.; Armstrong, N. R.; Kowalewski, T.; Pyun, J. Langmuir 2010, 26, 2083-2092.

(17) Cui, J.; Wang, A.; Edleman, N. L.; Ni, J.; Lee, P.; Armstrong, N. R.; Marks, T. J. Advanced Materials 2001, 2001, 9.

(18) Tahar, R. B. H.; Ban, T.; Ohya, Y.; Takahashi, Y. J. Journal of Applied Physics 1998, 83, 2631-2645.

(19) Armstrong, N. R.; Wang, W.; Alloway, D. M.; Placencia, D.; Ratcliff, E.; Brumbach, M. Macromolecules Rapid Communication 2009, 30, 717-731.

(20) Kugler, T.; Loegdlund, M.; Salaneck, W. R. Acc. Chem. Res. 1999, 32, 225-234.

(21) Koch, N. ChemPhysChem 2007, 8, 1438.

(22) Groenendaal, L. B.; Jonas, F.; Freitag, D.; Pielartzik, H.; Reynolds, J. R. Advanced Materials 2000, 12, 481-494.

(23) Berlin, A.; Vercelli, B.; Zotti, G. Polymer Review 2008, 48, 493-530.

(24) Doherty, W. J.; Wysocki, J. R.; Armstrong, N. R.; Saavedra, S. S. Macromolecules 2006, 39, 4418-4424. 
(25) Snaith, H. J.; Whiting, G. L.; Sun, B.; Greenham, N. C.; Huck, W. T. S.; Friend, R. H. Nano Letters 2005, 5, 1653-1657.

(26) Sakakiyama, T.; Ohkita, H.; Ohoka, M.; Ito, S.; Tsujii, Y.; Fukuda, T. Chem. Lett. 2005, 34, 1366-1367.

(27) Nardes, A. M.; Kemerink, M.; Janssen, R. A. J.; Bastiaansen, J. A. M.; Kiggen, N. M. M.; Langeveld, B. M. W.; Breemen, A. J. J. M. v.; Kok, M. M. d. Advanced Materials 2007, 19, 1196-1200.

(28) Kemerink, M.; Duren, J. K. J. v.; Jonkheijm, P.; Pasveer, W. F.; Koenraad, P. M.; Janssen, R. A. J.; Salemink, H. W. M.; Wolter, J. H. Nano Letters 2003, 3, 11911196.

(29) Kemerink, M.; Timpanaro, S.; Kok, M. N. d.; Meulenkamp, E. S.; Touwslager, F. J. Journal of Physical Chemistry B 2004, 108, 18820.

(30) Shallcross, R. C.; D'Ambruoso, G. D.; Korth, B. D.; Jr., H. K. H.; Zheng, Z.; Pyun, J.; Armstrong, N. R. Journal of the American Chemical Society 2007, 129, 11310-11311. 


\section{Preparation of Functional Semiconductor/Metal Nanostructures}

\subsection{Introduction}

\subsubsection{Motivation}

The burgeoning field of nanotechnology presents promising avenues for solving the dire need for development of clean and efficient energy production techniques. ${ }^{1}$ The capture, conversion, and transport of energy in nanomaterials are fundamentally different than that of bulk materials due to quantum size effects becoming dominant over classical effects. Current research in the area of nanoscience for energy applications is directed toward tuning these nanoscale effects of a variety of materials for inclusion in energy production technologies. ${ }^{1,2}$

The photocatalytic splitting of water for hydrogen production is a viable source for a clean, renewable fuel. ${ }^{2}$ The successful development of this technology hinges on the creation of a stable semiconductor system with an appropriate band gap and electron affinity for visible light absorption that can drive the redox chemistry necessary for hydrolysis. Additionally, it is necessary that the system be capable of separating photoinduced charge carriers such as excitons for lengths of time longer than is required for desired chemical reactions to occur from interaction with the resulting excited state of the system. ${ }^{2}$ The creation of a systematic method to alter the spatial arrangement on the nanoscale of various semiconductor and metal components capable of facilitating the desired photocatalyzed redox chemistries and charge separation of photoinduced charge carriers necessary is a crucial step towards a practical photocatalytic hydrogen production system. 


\subsubsection{Semiconductor/Metal Nanoparticle Synthesis}

There are numerous synthetic routes available for the preparation of nanoparticles including gas, liquid, and solid phase reactions. ${ }^{3}$ One of the most common methods and consequently the one of interest to this study is a liquid phase colloidal method. The first step of this process involves the rapid thermal decomposition of precursor compounds into reactive molecular species. These reactive molecular species then participate in a nucleation event where particles will begin their growth process. ${ }^{3}$ This step requires the presence of surfactants or ligands in solution that can capture nucleated particles and prevent them from precipitating out of solution prior to growth. The interaction of these molecules with the growing particle is very dynamic in the sense that they readily bind and release. ${ }^{4}$ This situation is amplified at high temperature and is desirable for the growth process as it allows for more of the reactive molecular species to penetrate the coating of surfactants and add to the growing particle. They also function to pacify and stabilize the particles when they are fully formed. ${ }^{4}$

Relatively high temperatures of $300{ }^{\circ} \mathrm{C}$ and above are required for the successful rapid decomposition of the precursor compounds. These high temperatures also serve to separate particle nucleation from the subsequent growth process, a desired effect for creating monodisperse particles via kinetic control. If the precursors are introduced expeditiously to the high temperature system the process of nucleation will initially be kinetically favored over that of growth, ensuring that all particles will begin growing at the same time. ${ }^{5}$ 


\subsubsection{Cadmium Sulfide/Platinum Photochemical System}

Cadmium Sulfide/Platinum (CdS/Pt) exhibits an appropriate band gap and possesses the ability to drive hydrogen evolution. Previously, random arrangements of the metal and semiconductor components of the system were investigated but with the current synthetic toolset available for the creation of nanoparticles composed of such materials with highly tunable structures, the opportunity to create an optimized photocatalytic hydrogen producing material is presented. ${ }^{2}$ In addition to the $\mathrm{CdS} / \mathrm{Pt}$ system which can facilitate hydrogen evolution, the incorporation of a cobalt oxide moiety would introduce functionality to the system capable of facilitating hydrolysis and thus a complete photocatalyzed water-splitting mechanism capable of hydrogen fuel (and oxygen) production.

\subsubsection{Quantum Confinement}

The effect of quantum confinement explains the phenomenon of size dependant electronic and optical properties of nanoparticle composed of semiconductor materials. ${ }^{6}$ In a bulk semiconductor material the electron-hole pair of an exciton is bound within a characteristic length termed the exciton Bohr radius. In this state the material exhibits an inherent band gap and continuous energy state. When the size domain of the semiconductor is reduced down to the critical quantum measurement of the exciton Bohr radius the effects of quantum confinement are observed and the energy state of the material transitions from a spectrum to discrete energy levels. As a result the bandgap of the material becomes size dependent, increasing with greater confinement of the exciton. ${ }^{6}$ 
Nanoparticles capable of spatially confining an exciton are found to exhibit electronic and optical properties that reside somewhat intermediate between bulk semiconducting materials and that of discrete molecules. The size and shape of the individual semiconductor crystal dictates these unique electronic properties. As the size of the crystal is reduced a greater energy difference between the highest valence band and lowest conduction band, a larger band gap, results. This means that more energy is required to excite the system and subsequently that more energy is released when it relaxes to a resting state. ${ }^{7}$

Quantum dots are semiconducting nanoparticles that are spherical. The spherical nature of these particles results in quantum confinement occurring in all three dimensions. Similarly, structures termed quantum wires facilitate quantum confinement in two dimensions while allowing free propagation in the third and structures referred to quantum wells confine in a singular dimension while allowing free propagation in the other two.

\subsubsection{Experimental Strategy for the Assembly of Cobalt/Platinum Asymmetrically} Tipped, Cadmium Selenide Seeded, Cadmium Sulfide (Co-CdSe@CdS-Pt) Nanorods

The present study is concerned with the assembly of nanorods with a CoCdSe@CdS-Pt architecture. Such a structure would be able to effectively separate the charge carriers of an exciton by directing electron holes towards the CdSe seeded end of the nanostructure while transporting the electrons to the Pt tip., ${ }^{2,8}$ Figure 4.1 depicts the Co-CdSe@CdS-Pt nanorod structure and proposed photoinduced redox chemistries facilitated by the nanostructure. 


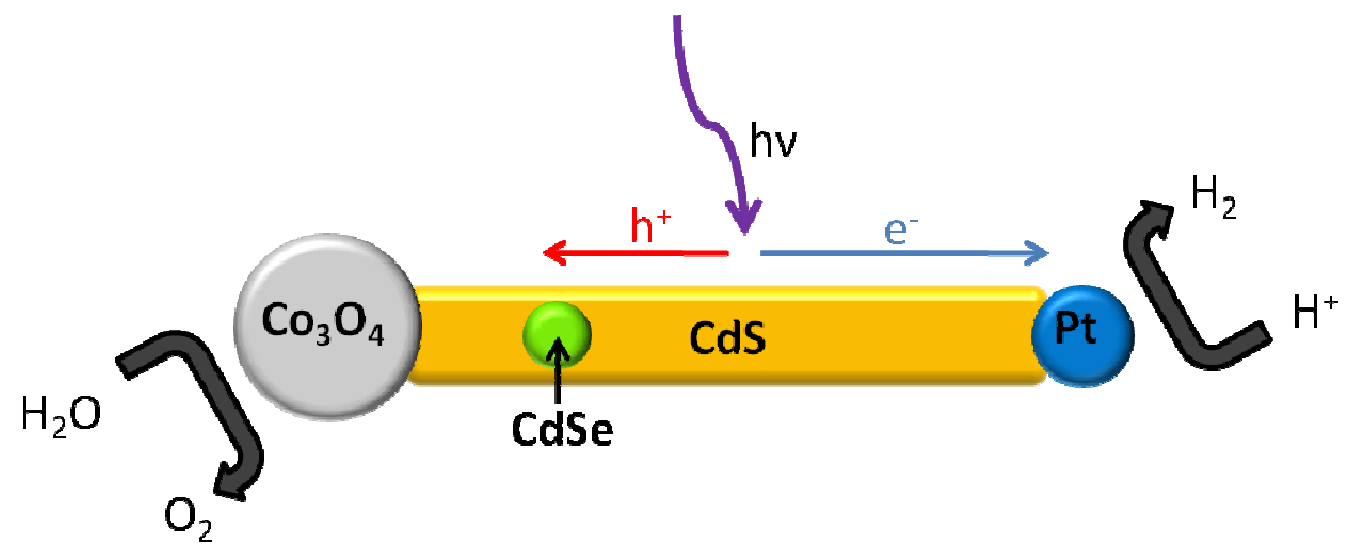

Figure 4.1 Schematic illustration of the Co-CdSe@CdS-Pt nanorod structure illustrating theoretical photoinduced redox chemisty.

In the present study CdSe quantum dots are synthesized via a hot injection synthetic method. The CdSe quantum dots are utilized as seeds for the growth of CdS nanorods. Proper modification of the synthetic parameters employed during the assembly of the nanorods should allow for the development of a facile procedure capable of creating structures with a tunable length. It is demonstrated that varying the ratio of quantum dot seeds to $\mathrm{Cd}$ and $\mathrm{S}$ precursor ultimately provides a synthetic strategy for synthesizing nanorods of relatively specific lengths with a good deal of reproducabiliy, a feature lacking of previously reported methods. Finally platinum nanoparticle tips can be incorporated into the nanohetero structure with hopes of future implementation as catalytic hydrogen producing constituent.

\subsection{Results and Discussion}

\subsubsection{CdSe Quantum Dot Synthesis and Characterization}

Scheme 4.1 displays the synthetic conditions employed in the preparation of CdSe quantum dots (QDs). Cadmium precursor $(\mathrm{CdO})$ was first dissolved in 
combination of octadecylphosphonic acid (ODPA) and trioctylphosphine oxide (TOPO) surfactants at high temperature $\left(>300^{\circ} \mathrm{C}\right)$. An aliquot of trioctylphosphine (TOP) was then added and the solution was heated to the desired temperature for introduction of the Se which was rapidly introduced into the hot mixture of $\mathrm{Cd}$ precursor to ensure homogenous nucleation of the CdSe nanoparticles. Varying the temperature at which the Se was introduced as well as the reaction time at elevated temperatures was found to create $\mathrm{CdSe}$ nanoparticles with varied optical properties as is in agreement with the literature $^{10}$ and is explained by the interplay between the kinetics of particle growth and nucleation as previously discussed. Transmission electron microscopy (TEM) and fluorescence spectroscopy were utilized to characterize the size of QDs. Figure 4.2 displays TEM micrographs of $\mathrm{CdSe}$ QDs drop cast onto a $\mathrm{Cu}$ from toluene. Figure 4.2a corresponds to a sample of yellow-green QDs prepared by removing the heating source from the reaction vessel immediately after the introduction of the Se precursor which resulted in particles with a diameter of $\sim 3.8 \mathrm{~nm}$ as estimated via TEM. Figure $4.2 \mathrm{~b}$ corresponds to a sample of blue-green QDs

Scheme 4.1 Preparation of CdSe Quantum Dots

$\mathrm{CdO}+\mathrm{Se}$ TOPO, ODPA, TOP 375 deg. C

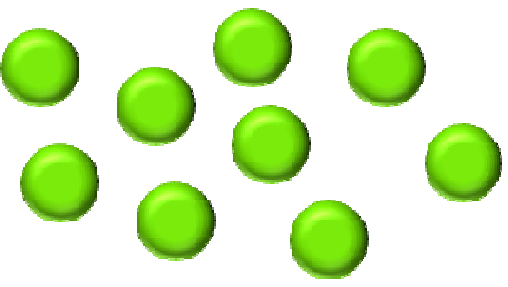



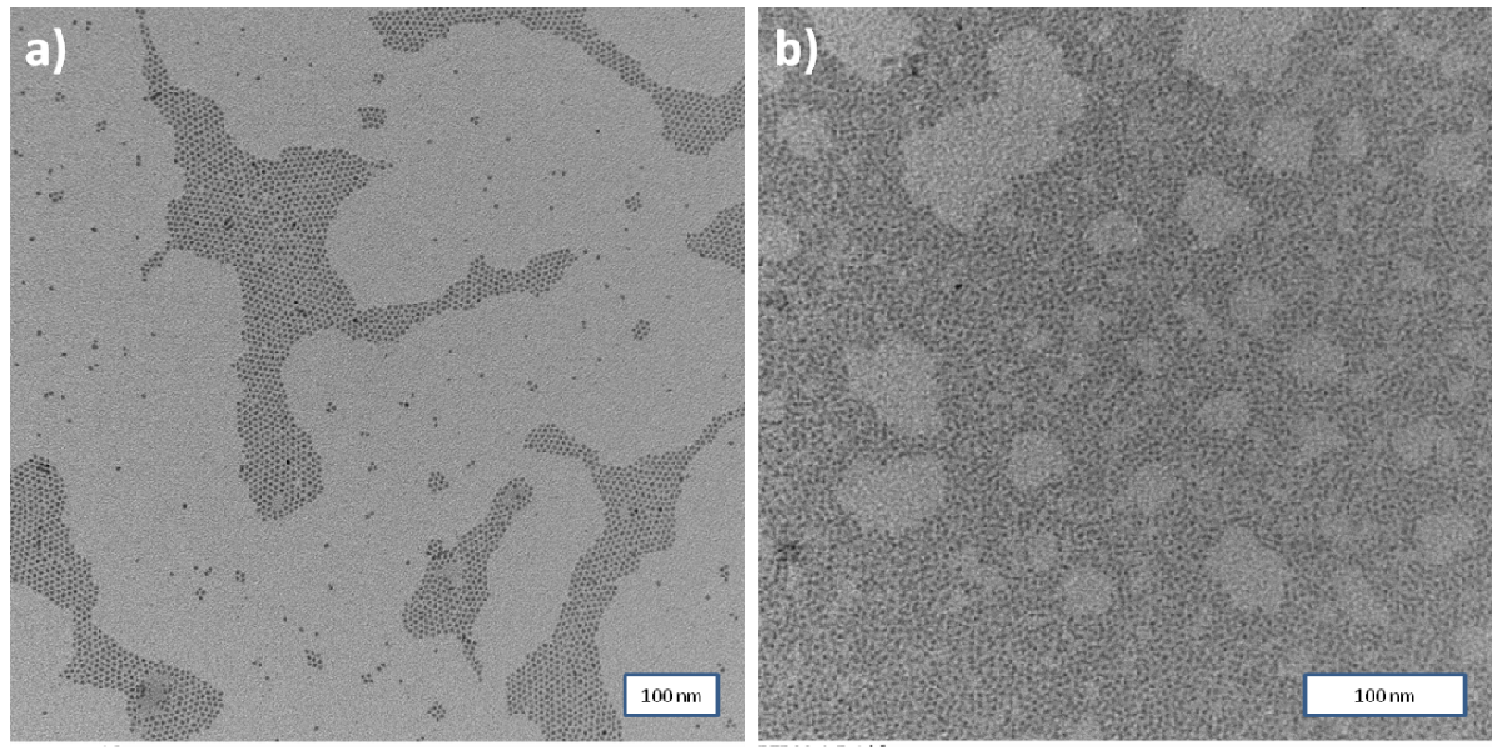

Figure 4.2 TEM micrographs of CdSe QDs with diameters of a) $\sim 3.8 \mathrm{~nm}$ and b) $\sim 2.6 \mathrm{~nm}$

prepared by introducing the Se precursor to the system with the heating source already removed and a near simultaneous introduction of chilled octadecene, added in order to bring the temperature of the reaction below a temperature sufficient to propagate particle growth almost immediately after nucleation. This resulted in particles with a diameter of $\sim 2.6 \mathrm{~nm}$ as estimated via TEM. Figure 4.3 displays the fluorescence of the two samples.

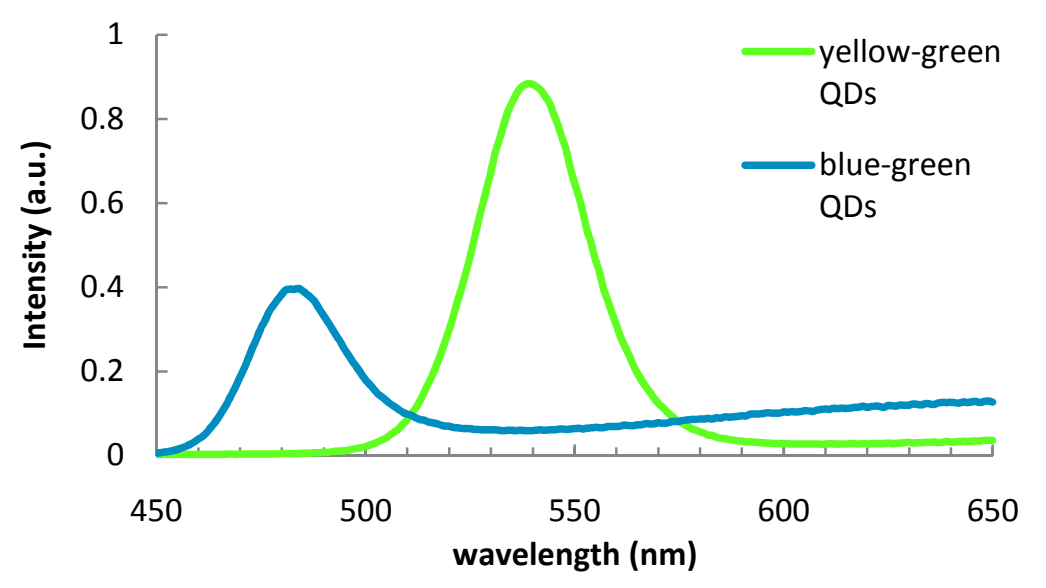

Figure 4.3 Fluorescence spectra of the blue-green QDs and yellow-green QDs. 
4.2.2 Cadmium Selenide Seeded Cadmium Sulfide (CdSe@CdS) Nanorod Synthesis and Characterization

Scheme 4.2 displays the synthetic conditions used for the preparation of CdSe@CdS nanorods. Similar to the preparation of the CdSe seeds, CdO was dissolved in a mixture of surfactants at high temperature. A mixture of S and CdSe dots was then rapidly introduced into the hot mixture $\left(\sim 370^{\circ} \mathrm{C}\right)$ of $\mathrm{CdO}$ and surfactants and the reaction was allowed to recover to high temperature and incubate. A fairly exhaustive investigation on the effects of varying the ration of different components of the synthetic system showed that the ratio of QD seeds to precursor materials had a notable effect on the length of the resulting CdSe@ CdS nanorods as shown in Table 4.1 where sample numbers refer to the complete table in the experimental section. The effects of $\mathrm{CdO}$ precursor amounts (Table 4.2) and injection temperature (Table 4.3) on nanorod length were also investigated but no notable effects were observed.

Transmission electron microscopy was utilized for characterizing the $\mathrm{CdSe} @ \mathrm{CdS}$ nanorods. Figure 4.4 shows TEM micrographs of shorter nanorods with a length of $27.822 \pm 0.981 \mathrm{~nm}$ and longer nanorods with a length of $105.2 \pm 15.2 \mathrm{~nm}$ are shown by Figure 4.5.

Scheme 4.2 Preparation of CdSe@CdS Nanorods

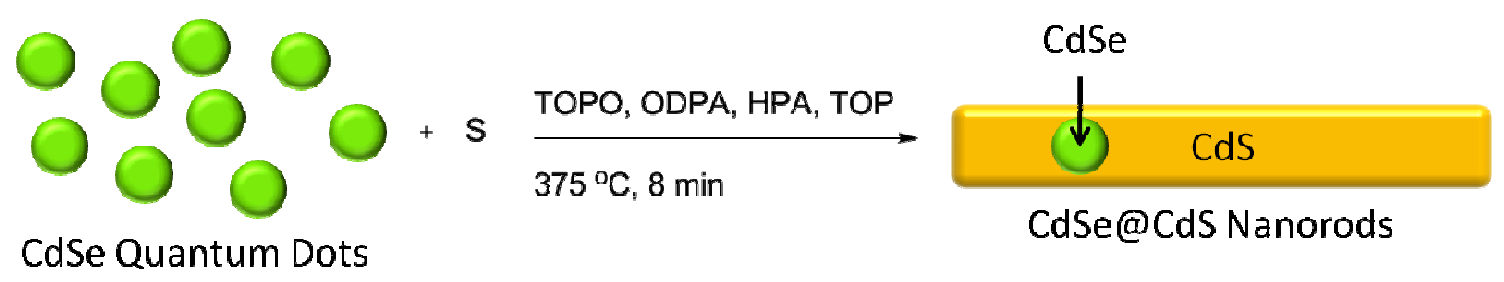


Table 4.1 Synthetic Conditions With Varied QD concentrations for Preparation of CdSe@CdS Nanorods ${ }^{a}$

\begin{tabular}{|c|c|c|c|c|c|c|}
\hline Sample & $\mathrm{T}_{\text {inject }}\left({ }^{\circ} \mathrm{C}\right)$ & $\mathrm{S}(\mathrm{mg})$ & $\mathrm{CdO}(\mathrm{mg})$ & $\mathrm{QD}(\mathrm{mg})$ & \multicolumn{2}{|c|}{$\mathrm{L}(\mathrm{nm})$} \\
\hline $19^{b}$ & 350 & 33.3 & 75 & 4.56 & $24.4 \pm 1.3$ \\
\hline 20 & 350 & 33.3 & 75 & 2.28 & $36.0 \pm 1.9$ \\
\hline 21 & 350 & 33.3 & 75 & 1.39 & $70.1 \pm 9.6$ \\
\hline 22 & 350 & 33.3 & 75 & 1.11 & $62.1 \pm 3.3$ \\
\hline 23 & 350 & 33.3 & 75 & 1.11 & $56.4 \pm 20.0$ \\
\hline 24 & 350 & 33.3 & 75 & 0.83 & $46.2 \pm 12.2$ \\
\hline 25 & 350 & 33.3 & 75 & 0.56 & $136.7 \pm 3.3$ \\
\hline 26 & 350 & 33.3 & 75 & 0.56 & $105.2 \pm 15.2$ \\
\hline 27 & 350 & 33.3 & 75 & 0.28 & $62.3 \pm 16.0$ \\
\hline
\end{tabular}

${ }^{a}$ All samples also were prepared with $3.0 \mathrm{~g}$ TOPO, $80 \mathrm{mg}$ HPA and $290 \mathrm{mg}$ ODPA

${ }^{b}$ Prepared with $40 \mathrm{mg}$ HPA

Table 4.2 Synthetic Conditions With Varied CdO concentrations for Preparation of CdSe@CdS Nanorods ${ }^{a}$

\begin{tabular}{|c|c|c|c|c|c|c|c|}
\hline Sample & $\mathrm{T}_{\text {inject }}$ & $\mathrm{S}(\mathrm{mg})$ & $\mathrm{CdO}$ & QD & \multicolumn{3}{|c|}{$\mathrm{L}(\mathrm{nm})$} \\
\hline 16 & 375 & 33.3 & 60 & 4.56 & 33.3 & \pm & 8.7 \\
\hline 17 & 375 & 33.3 & 90 & 4.56 & 27.8 & \pm & 1.0 \\
\hline 18 & 375 & 33.3 & 110 & 4.56 & 35.5 & \pm & 3.2 \\
\hline
\end{tabular}

${ }^{a}$ All samples also were prepared with $3.0 \mathrm{~g}$ TOPO, $80 \mathrm{mg}$ HPA and $290 \mathrm{mg}$ ODPA

Table 4.3 Synthetic Conditions With Varied S:TOP Injection Temperatures for Preparation of CdSe@CdS Nanorods ${ }^{a}$

\begin{tabular}{|c|c|c|c|c|c|c|c|}
\hline Sample & $\mathrm{T}_{\text {inject }}$ & $\mathrm{S}(\mathrm{mg})$ & $\mathrm{CdO}$ & QD & \multicolumn{3}{|c|}{$\mathrm{L}(\mathrm{nm})$} \\
\hline 13 & 350 & 33.3 & 75 & 4.78 & 32.5 & \pm & 4.9 \\
\hline 14 & 360 & 33.3 & 75 & 4.78 & 31.5 & \pm & 1.9 \\
\hline 15 & 370 & 33.3 & 75 & 4.78 & 32.2 & \pm & 2.3 \\
\hline
\end{tabular}

${ }^{a}$ All samples also were prepared with $3.0 \mathrm{~g}$ TOPO, $80 \mathrm{mg}$ HPA and $290 \mathrm{mg}$ ODPA 



Figure 4.4 TEM microcraph of nanorods with a length of $27.82 \pm 0.98 \mathrm{~nm}$ (Sample 17)


Figure 4.5 TEM microcraph of nanorods with a length of $105.2 \pm 15.2 \mathrm{~nm}$ (Sample 26) 


\subsubsection{Platinum Tipped Cadmium Selenide Seeded Cadmium Sulfide (CdSe@CdS-Pt)}

Nanorod Synthesis and Characterization

Scheme 4.3 illustrates the synthetic conditions employed in the assembly of CdSe@CdS-Pt nanorods. A suspension of Pt precursor $\left(\mathrm{Pt}^{\mathrm{II}}(\mathrm{acac})_{2}\right)$ and $\mathrm{CdSe} @ \mathrm{CdS}$ nanorods was injected in a hot solution of surfactants suitable for interaction with Pt. Pt nanoparticles were nucleated on the tip of the nanorods subsequent to thermolysis of the Pt precursor and then allowed to grow. TEM was used to confirm the addition of the Pt tips to the CdSe@CdS nanorods as shown in Figure 4.6

Scheme 4.3 Assembly of CdSe@CdS-Pt Nanorods
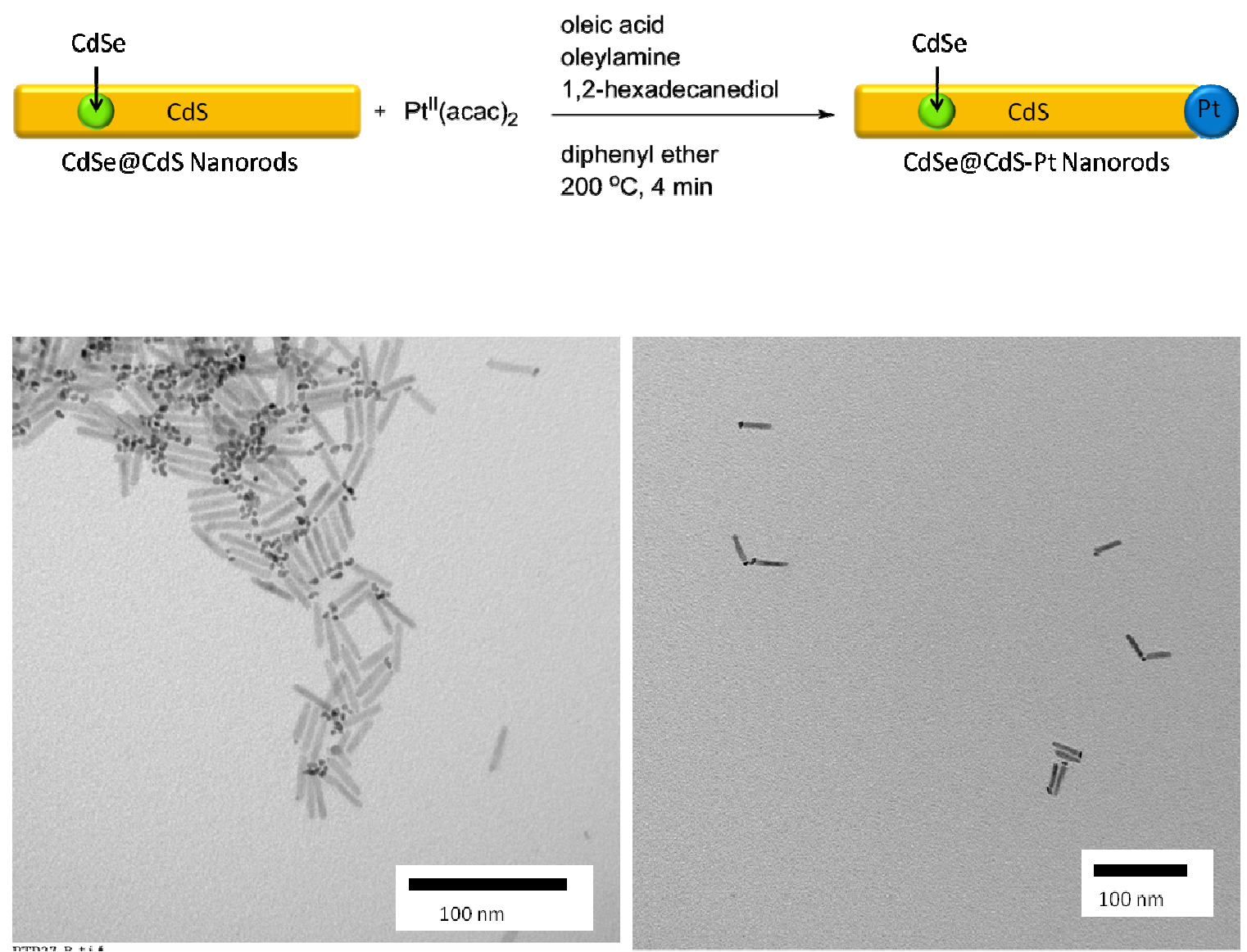
Figure 4.6 TEM micrograph of CdSe@CdS-Pt nanorods where the dark circles are indicitave of the Pt tips

\subsection{Conclusions}

CdSe@CdS-Pt nanorods were successfully produced via a stepwise assembly method. CdSe quantum dots were first synthesized and then utilized as seeds for the growth of CdSe@CdS nanorods. A facile method for tuning the length of the nanorods was developed by varying the ratio of QD seeds to CdS precursors. Pt tips were then added to the nanoheterostructure via thermolysis of a Pt precursor and subsequent nucleation of Pt nanoparticles on the end of the CdSe@CdS nanorods. TEM was utilized for charactizing the size of the various particles produced throughout the assembly process and to confirm the successful addition of the Pt tips. Work is currently under way for the addition of an additional Co tip to the structure with hopes of creating a nanostructure capable of photocatalytic water splitting.

\subsection{Experimental}

\subsubsection{Materials}

All materials were acquired from commercially available sources and utilized without further purification.

\subsubsection{Instrumentation}

Transmission electron microscopy was completed utilizing a Phillips CM12 transmission electron microscope and Cu TEM grids (hexagonal, 300 mesh) at $80 \mathrm{keV}$. Fluorescence spectroscopy was completed on a PTI fluorimeter in toluene at $305 \mathrm{~nm}$. 


\subsubsection{Nanoparticle Synthesis}

\subsubsection{CdSe Quantum Dot Synthesis}

A $50 \mathrm{~mL}$ 3-necked round bottom flask equipped with a stirbar and reflux condenser was loaded with trioctylphosphine oxide (TOPO, $3.0 \mathrm{~g}$ ), octadecylphosphonic acid (ODPA, $0.29 \mathrm{~g})$ and $\mathrm{CdO}(0.060 \mathrm{~g})$. The flask was heated to $150^{\circ} \mathrm{C}$ and put under vacuum for 1 hour to remove any trace water. The flask was then heated to $300^{\circ} \mathrm{C}$ and trioctylphosphine (TOP, $1.8 \mathrm{~mL}$ ) was injected under Ar. The temperature was then increased to $\sim 370{ }^{\circ} \mathrm{C}$ and a Se:TOP solution (0.058g Se in $1.8 \mathrm{~mL}$ TOP) was rapidly injected into the rapidly stirried solution. The reaction time was varied to manipulate the size of the resulting particles but typically the heating source was removed immediately

after Se:TOP injection and the reaction cooled which yields green fluorescent CdSe dots. The particles were isolated via precipitation/redissolution in EtOH/Toluene and characterized via TEM and fluorescence spectroscopy.

\subsubsection{CdSe@CdS Nanorod Synthesis}

A $50 \mathrm{~mL}$ 3-necked round bottom flask equipped with a stirbar and reflux condenser was loaded with TOPO (3 g), ODPA (0.29 g), HPA (0.080 g) and CdO (0.060 g). The flask was heated to $150^{\circ} \mathrm{C}$ and put under vacuum for 1 hour to remove any trace water. The flask was then heated to $300{ }^{\circ} \mathrm{C}$ and TOP $(1.8 \mathrm{~mL})$ was injected under Ar. The temperature was then increased to $350{ }^{\circ} \mathrm{C}$ and $\mathrm{CdSe}$ quantum dot solution in TOP $(1.8 \mathrm{~mL})$ was rapidly injected into the rapidly stirried solution. The reaction was stirred 
for 6-8 min after which the heating mantle was removed and the reaction mixture was cooled. The particles were isolated via precipitation/redissolution in EtOH/Toluene and characterized via TEM. Table 4.4 displays all modifications to the amounts of various reagents utilized in various synthetic preparations

Table 4.4 Synthetic Conditions for Various Preparations of CdSe@CdS Nanorods ${ }^{a}$

\begin{tabular}{|c|c|c|c|c|c|c|c|}
\hline \multirow{2}{*}{$\begin{array}{c}\text { Sample } \\
1\end{array}$} & \multirow{2}{*}{$\frac{\mathrm{T}_{\text {inject }}\left({ }^{\circ} \mathrm{C}\right)}{350}$} & \multirow{2}{*}{$\frac{\mathrm{S}(\mathrm{mg})}{66.7}$} & \multirow{2}{*}{$\frac{\mathrm{CdO}(\mathrm{mg})}{60}$} & \multirow{2}{*}{$\frac{\mathrm{QD}(\mathrm{mg})}{5.56}$} & \multicolumn{2}{|c|}{$\mathrm{L}(\mathrm{nm})$} & W (nm) \\
\hline & & & & & 28.2 & \pm 4.0 & $5.64 \pm 1.15$ \\
\hline 2 & 350 & 66.7 & 60 & 5.56 & 27.5 & \pm 2.7 & $5.54 \pm 0.60$ \\
\hline 3 & 350 & 66.7 & 86 & 5.56 & 32.0 & \pm 3.0 & $5.00 \pm 1.00$ \\
\hline 4 & 350 & 66.7 & 86 & 5.56 & 28.8 & \pm 3.2 & $6.12 \pm 0.89$ \\
\hline 5 & 350 & 66.7 & 91 & 5.56 & 30.5 & \pm 5.1 & $7.80 \pm 0.89$ \\
\hline 6 & 350 & 66.7 & 60 & 5.56 & 25.8 & \pm 4.6 & $4.96 \pm 0.45$ \\
\hline 7 & 350 & 66.7 & 86 & 5.56 & 29.6 & \pm 3.2 & $5.72 \pm 0.76$ \\
\hline 8 & 350 & 33.3 & 86 & 4.78 & 37.7 & \pm 2.1 & $4.77 \pm 0.74$ \\
\hline 9 & 350 & 33.3 & 60 & 4.78 & 26.4 & \pm 4.2 & $5.01 \pm 0.69$ \\
\hline 10 & 350 & 33.3 & 75 & 4.78 & 39.2 & \pm 4.1 & $4.51 \pm 0.65$ \\
\hline 11 & 350 & 33.3 & 75 & 4.78 & 36.3 & \pm 1.0 & $4.98 \pm 0.54$ \\
\hline 12 & 350 & 33.3 & 93 & 4.78 & 28.6 & \pm 1.5 & $5.00 \pm 0.65$ \\
\hline 13 & 350 & 33.3 & 75 & 4.78 & 32.5 & \pm 4.9 & $5.13 \pm 0.66$ \\
\hline 14 & 360 & 33.3 & 75 & 4.78 & 31.5 & \pm 1.9 & $5.09 \pm 0.53$ \\
\hline 15 & 370 & 33.3 & 75 & 4.78 & 32.2 & \pm 2.3 & $4.62 \pm 0.59$ \\
\hline 16 & 375 & 33.3 & 60 & 4.56 & 33.3 & \pm 8.7 & $8.28 \pm 1.16$ \\
\hline 17 & 375 & 33.3 & 90 & 4.56 & 27.8 & \pm 1.0 & $4.06 \pm 0.57$ \\
\hline 18 & 375 & 33.3 & 110 & 4.56 & 35.5 & \pm 3.2 & $4.43 \pm 0.57$ \\
\hline $19^{\mathrm{b}}$ & 350 & 33.3 & 75 & 4.56 & 24.4 & \pm 1.3 & $4.56 \pm 0.81$ \\
\hline 20 & 350 & 33.3 & 75 & 2.28 & 36.0 & \pm 1.9 & $4.59 \pm 5.27$ \\
\hline 21 & 350 & 33.3 & 75 & 1.39 & 70.1 & \pm 9.6 & $\mathrm{NA} \pm \mathrm{NA}$ \\
\hline 22 & 350 & 33.3 & 75 & 1.11 & 62.1 & \pm 3.3 & $6.26 \pm 0.45$ \\
\hline 23 & 350 & 33.3 & 75 & 1.11 & 56.4 & \pm 20.0 & $\mathrm{NA} \pm \mathrm{NA}$ \\
\hline 24 & 350 & 33.3 & 75 & 0.83 & 46.2 & \pm 12.2 & $\mathrm{NA} \pm \mathrm{NA}$ \\
\hline 25 & 350 & 33.3 & 75 & 0.56 & 136.7 & \pm 3.3 & $6.70 \pm 0.99$ \\
\hline 26 & 350 & 33.3 & 75 & 0.56 & 105.2 & \pm 15.2 & $\mathrm{NA} \pm \mathrm{NA}$ \\
\hline 27 & 350 & 33.3 & 75 & 0.28 & 62.3 & \pm 16.0 & $\mathrm{NA} \pm \mathrm{NA}$ \\
\hline
\end{tabular}

${ }^{a}$ All samples also were prepared with $3.0 \mathrm{~g}$ TOPO, $80 \mathrm{mg}$ HPA and $290 \mathrm{mg}$ ODPA

${ }^{b}$ Prepared with $40 \mathrm{mg}$ HPA 


\subsubsection{Pt tipping of CdSe@CdS Nanorods}

A $50 \mathrm{~mL}$ three-neck flask equipped with a magnetic stirbar and reflux condenser was loaded with oleic acid (0.2 mL), oleylamine (0.2 mL), 1,2-hexadecanediol (43.0 mg) and diphenyl ether $(10 \mathrm{~mL})$ and heated to $80{ }^{\circ} \mathrm{C}$ under vacuum for $30 \mathrm{~min}$ to remove traces of water. Pt acetylacetonate $(\sim 20 \mathrm{mg})$ was added to a suspension of $\mathrm{CdSe} / \mathrm{CdS}$ rods (about half of the synthesis product) in dichlorobenzene ( $1 \mathrm{ml})$ and the solution was sonicated for a few seconds to promote dissolution of the Pt precursor. Under argon, the mixture of surfactants and diphenyl ether was heated to $200^{\circ} \mathrm{C}$. At this point the $\mathrm{Pt}$ precursor and seeded rods in dichlorobenzene were injecting into the flask. Upon temperature recovery to $200{ }^{\circ} \mathrm{C}$ the solution turned black. After several minutes the heating mantle was removed and the solution cooled to room temperature. The particles were isolated via precipitation/redissolution in $\mathrm{EtOH} / \mathrm{Toluene}$ and characterized via TEM.

\subsection{References}

(1) Li, Y.; Somorjai, G. A. Nano Letters 2010, 10, 2289-2295.

(2) Amirav, L.; Alivisatos, A. P. Journal of Physical Chemistry Letters 2010, 1.

(3) Nagarajan, R.; Hatton, T. A. American Chemical Society Symposium Series 2008.

(4) Handbook of Nanophysics: Nanoparticles and Quantum Dots; Sattler, K. D., Ed.; CRC Press: Boca Raton FL, 2011.

(5) Alivisatos, A. P. Science 1996, 271, 933-937.

(6) Brus, L. Applied Physics A: Solids and Surfaces 1991, A53, 465-74.

(7) Alivisatos, A. P. Journal of Physical Chemistry 1996, 100, 13226-13239.

(8) Kolasinkski, K. W. Surface Science: Foundations of Catalysis and Nanoscience; John Wiley \& Sons: Chichester, United Kingdom, 2008. 
(9) Deka, S.; Falqui, A.; Bertoni, G.; Sangregorio, C.; Poneti, G.; Morello, G.; Giorgi, M. D.; Giannini, C.; Cingolani, R.; Manna, L.; Cozzoli, P. D. Journal of the American Chemical Society 2009, 131, 12817-12828.

(10) Carbone, L.; Nobile, C.; Giorgi, M. D.; Sala, F. D.; Morello, G.; Pompa, P.; Hytch, M.; Snoeck, E.; Franchini, I. R.; Nadasan, M.; Silvestre, A. F.; Kudera, S.; Cingolani, R.; Krahne, R.; Manna, L. Nano Letters 2007, 7, 2942-2950. 


\section{Conclusions}

Reversible Diels-Alder chemistry was utilized to manipulate the surface energy of

glass substrates. Hydrophobic dieneophiles were prepared and attached to glass slides and capillaries to yield a non-wetting surface. Thermal treatment of the surfaces cleaved the Diels-Alder linkage, and resulted in the fabrication of a hydrophilic surface. Preliminary analysis utilized contact angle (CA) measurements to monitor the change in surface energy, and observed a hydrophilic state $\left(\mathrm{CA}-70 \pm 3^{\circ}\right)$ before attachment of the dieneophile to a hydrophobic state $\left(\mathrm{CA}-101 \pm 9^{\circ}\right)$ followed by regeneration of the hydrophilic state $\left(\mathrm{CA}-70 \pm 6^{\circ}\right)$ upon cleavage of the Diels-Alder linkage. The treatments were then applied to glass capillaries, with effective treatment confirmed by fluid column measurements. Patterned treatments were also demonstrated to provide effective flow gating. Finally, attempts to create self-pressurizing capillaries were unsuccessful due to pronounced contact angle hysteresis for the hydrophobic surface treatment.

Successive surface initiated atom transfer radical polymerization and electropolymerization was utilized to functionalize indium-tin oxide substrates. A novel hybrid styrenic/thiophene monomer, ProDOT-Sty, was synthesized that allowed for the templation of electropolymerized poly(3,4-alkyleneoxythiophene) brushes by first creating a poly(styrene) backbone via a controlled radical polymerization process. Cyclic voltammetry of an ITO electrode functionalized with poly(ProDOT-Sty) brushes grafted from the ITO surface via SI-ATRP gave clear indication of the electropolymerization event beginning at approximately $+0.7 \mathrm{~V}$ vs $\mathrm{Fc} / \mathrm{Fc}^{+}$. Photo patterning of the phosphonic acid ATRP initiator immobilized on the ITO surface via lithography with ultraviolet light was confirmed via SEM and allowed for the creation of a surface that would limit growth 
of the polymer species to a patterned area for facile film brush thickness characterization via $\mathrm{AFM}$ at a later time.

A platinum tipped, cadmium sulfide nanorod with cadmium selenide quantum dot core was assembled. CdSe quantum dots were prepared with various diameters by adjusting reaction temperatures and times. These dots were employed as seeds for the growth of CdS nanorods of various lengths ranging from $\sim 25 \mathrm{~nm}$ to $\sim 135 \mathrm{~nm}$. Investigation of the influence of the various synthetic conditions of the nanorod synthesis led to the conclusion that the ratio of CdSe seeds to $\mathrm{Cd}$ and $\mathrm{S}$ precursors could be manipulated in order to influence the length to which the nanorods grew. Pt tips were attached to the tip of the CdSe@CdS nanorods as theoretical photocatalytic hydrogen production sites. TEM was utilized to characterize the various nanoparticles at each stage of assembly. 